\title{
Design Polysaccharides of Marine Origin: Chemical Modifications to Reach Advanced Versatile Compounds
}

\author{
Nathalie Chopin ${ }^{1,2}$, Xavier Guillory ${ }^{1}$, Pierre Weiss ${ }^{3}$, Jean Le Bideau ${ }^{2}$ and Sylvia Colliec-Jouault ${ }^{1,{ }^{*}}$
}

\begin{abstract}
1 IFREMER Institut Français de Recherche pour l'Exploitation de la Mer, Laboratoire de Biotechnologie et Molécules Marines, Rue de l'île d'Yeu, BP 21105, 44311, Nantes Cedex 03, France.

${ }^{2}$ Institut des Matériaux Jean Rouxel - CNRS - Université de Nantes, 2 Rue de la Houssinière, B.P. 32229, 44322 Nantes Cedex 3, France.

3 INSERM, U791, Laboratoire d'Ingénierie Ostéo-Articulaire et Dentaire (LIOAD) - Université de Nantes, 1, place Alexis Ricordeau, BP 84215, 44042 Nantes Cedex 1, France.
\end{abstract}

*: Corresponding author : Sylvia Colliec-Jouault, tel.: +33 240374093 ; fax: +33 240374071 ;
e-mail address : sylvia.colliecjouault@ifremer.fr

\begin{abstract}
:
Polysaccharides are among the most abundant macromolecules on Earth. These polymers are easily obtained from various marine resources such as algae, microorganisms and crustacean shells. The structure of these natural carbohydrates is innovative and quite complex. Marine biopolymers represent key scaffolds toward large challenging fields, such as biomedical applications (glycosaminoglycans, regenerative medicine and drug delivery) and tailored biomaterials. Chemical modifications can be applied to modify their final properties in a specific purpose. New functional glycans are achievable and represent a real potential with their intrinsic biocompatibility and biodegradability.
\end{abstract}

Hydroxyl groups are ubiquitous in polysaccharides structure and involved in most of the chemical modifications. The most useful functionalities are ester, ether, amide, amine and alkyl groups. The starting materials could be a natural or depolymerized polymers and the reaction considered with a regioselective point of view.

In this review, we will focus on chitin polysaccharide, which is extracted according to industrial processing from exoskeleton of several marine crustaceans. A subsequent deacylation provides chitosan. This marine polysaccharide is very similar to cellulose, a widespread fiber plant organic polymer, except for an amine group on the C2 position instead of a hydroxyl group. Furthermore, seaweeds provide the most abundant sources of polysaccharides: alginates, agar/agarose, carrageenans and fucoidans. In order to improve the original physicochemical and biochemical properties, we will highlight the chemical modifications involving the listed marine polysaccharides of interest. 
Graphical abstract :
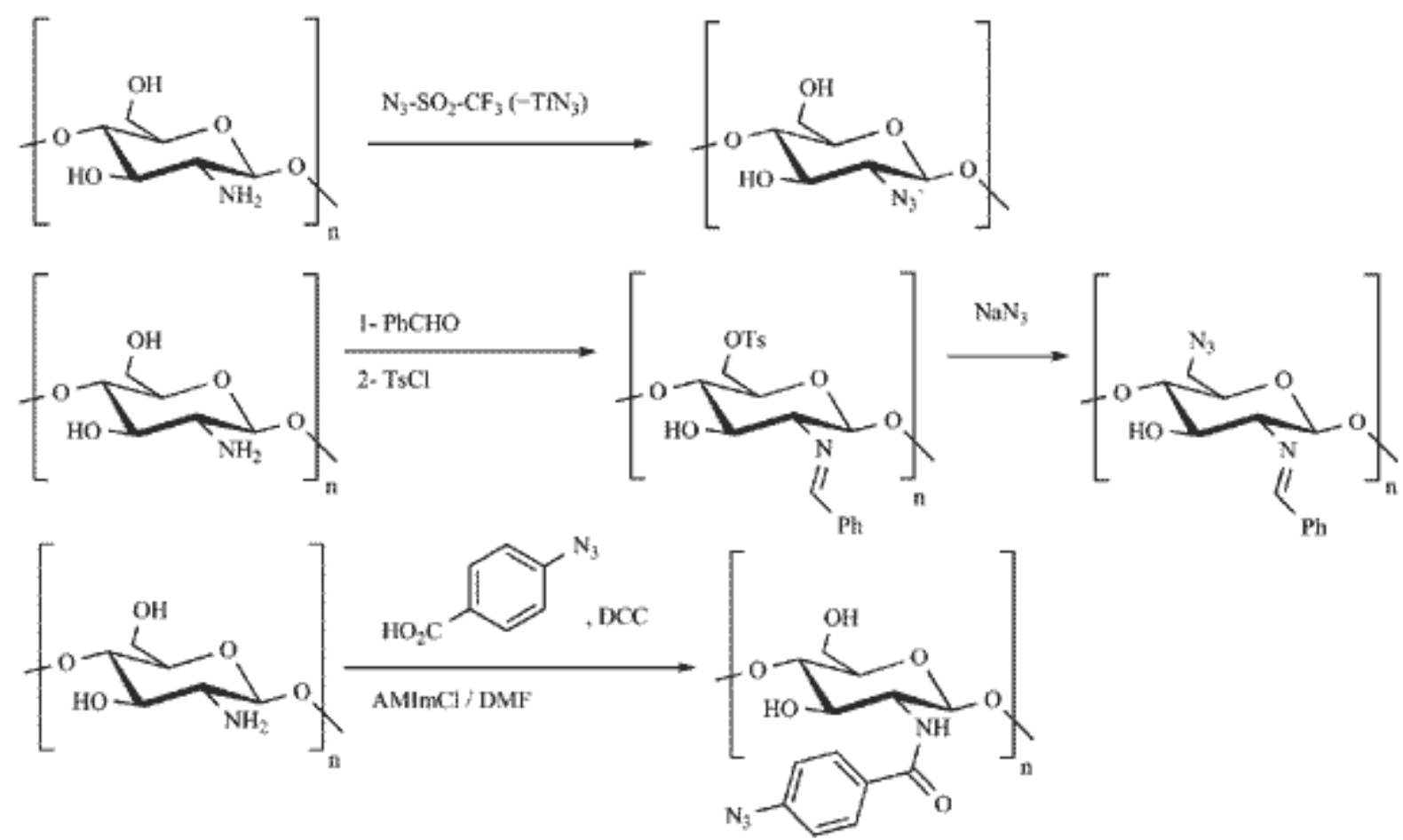

Keywords: Alginates ; carrageenans ; chemical modification ; chitin, chitosan ; grafting ; marine polysaccharide ; polymer 


\section{INTRODUCTION}

Polysaccharides are widespread macromolecules available from natural resources. Their structural diversity makes them attractive since they are not easily attainable via chemical synthesis. A striking advantage of polymeric carbohydrates is their inherent characteristics such as biocompatibility, biodegradability and nontoxicity towards living organisms. These natural molecules of interest can be considered as sustainable substances with the real improvements in biotechnological techniques reducing the resource consumption and increasing economic benefits. The development of the blue (or marine) biotechnologies is one of the key factors responsible of the rapid expansion of these biopolymers.

Depending on the final purpose of their utilization, natural properties of polysaccharides can be enhanced by structural modifications. In this review, we will focus on chemical modifications of typical marine polysaccharides bearing reactive functional groups. The introduction of a new reactive group will not change fundamentally the backbone of the macromolecules but will unlock advanced modifications required in specific applications. We will concentrate on the most representative polysaccharides extracted from marine environment: chitin and its derivative chitosan, alginates, carrageenans and agar. As the chemical structure of marine polysaccharides depends on various factors such as sources, seasons, environmental conditions and the method of extraction, we will consider here each generally admitted repeating unit with their most representative functional groups.
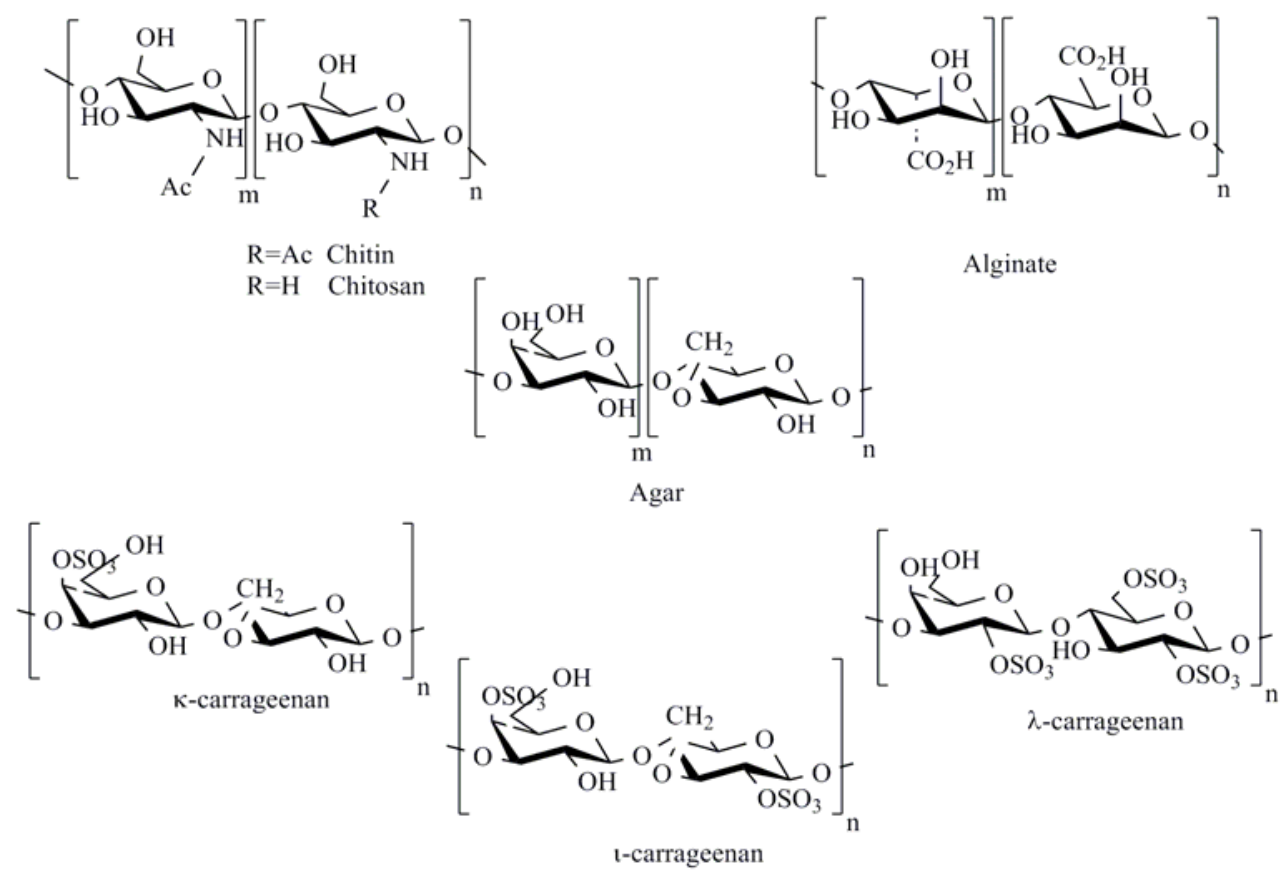

Fig. 1 : common marine polysaccharides 
First of all, chitin is the second most abundant polysaccharide just after cellulose. It is a linear high-molecular weight (HMW) biopolymer with a repeating unit constituted of $\beta$ - $(1 \rightarrow 4)$ linked $N$-acetylglucosamine (GlcNAc) residues: $\rightarrow 4)-\beta-D-G l c N p$ Ac- $(1 \rightarrow$ (Fig. 1). Three reactive chemical groups are present onto the chitin GlcNAc structure, which are primary and secondary hydroxyl groups and $N$-acetylated primary amine. Focusing on marine origin, chitin is found in several invertebrates as a structural component of the exoskeleton of arthropods such as crustaceans (e.g. shrimps, crabs, lobsters and shells) [1], and of the endoskeleton of mollusks. The crude chitin is a highly ordered crystalline molecule outlined with strong inter- and intramolecular hydrogen bonds. Depending on its sources, two allomorphs occur: abundant $\alpha$-chitin and rare $\beta$-chitin. The $\alpha$ form is defined by an antiparallel arrangement of the sugar chains, which induces a great stability of the structure. Whereas chains in $\beta$-chitin are packed in a parallel arrangement giving weaker intermolecular forces that leads to both higher reactivity and affinity towards solvents. We highlight here that solubility is an important factor that can restrict polysaccharide chemical modifications or not.

As a consequence, a wider range of chemical reactions was successfully applied to chitin in order to obtain the deacetylated chitin derivative: chitosan. Chitosan is thus composed of $\mathrm{N}$-acetylglucosamine residues and connected through $\beta-(1 \rightarrow 4)$ glycosidic linkage: $\rightarrow 4)-\beta-D-G l c N p A c-(1 \rightarrow 4)-\beta-D-G l c N p-(1 \rightarrow$ (Fig. 1$)$. The only difference between the two structures of chitin and chitosan is the deacetylation degree, i.e. the ratio of deacetylated units in the polymer chain. Chitosan is defined with a deacetylation degree between $50 \%$ and $90 \%$ or more generally, chitosan is soluble in dilute acetic acid solution below its pKa (e.g. 6.5) while chitin is not. The major difference of reactivity between these two similar compounds is the primary amino group bearing in the glucosamine units of chitosan, introducing a potential cationic nature with its protonated form.

Much attention has been dedicated to alginates which are quantitatively the major polysaccharides contained in the cell wall of brown algae (Phaeophyta). It is both a biopolymer and a polyelectrolyte. This unbranched anionic copolymer is composed of irregular sequences of mannuronic acid (M block) and its C5 epimer, guluronic acid (G block): $\rightarrow 4)-\beta-D-M a n p A-(1 \rightarrow 4)-\alpha-L-G u l p A-(1 \rightarrow$ (Fig. 1). These two blocks are different in the configuration of their carboxyl group which negative charge density could be tuned by $\mathrm{pH}$ as the alginic acid form is insoluble in water. The solubility of alginates depends on the M:G ratio and temperature. Alginates are able to form gels in the presence of bivalent ions, notably $\mathrm{Ca}^{2+}$. This marine macromolecule is the functional analogue of pectin, found into the higher plant. Poly-M sequences show linear and flexible conformation while 
poly-G sequences introduce steric hindrance around the carboxyl group. According to the types of brown seaweed, the seaweed age, the part of the plant (frond, stipe or anchor) but also the time of year, the M:G ratio varies in alginates and can affect the final composition and properties of alginate polysaccharide. Along the backbone, free hydroxyl groups and carboxyl groups could undergo different chemical modifications to synthetize relevant derivatives.

Another anionic polysaccharide of marine origin is carrageenan, generic name for a family of linear sulfated polymers extracted from species of red seaweeds (Rhodophyta). Their repeating unit is made up with two monomers derived from galactose. These hydrophilic galactans are classified according to the type of residue (e.g. G unit= 3- $\beta-\mathrm{D}$-galactopyranosyl, D-unit= 4- $\alpha-\mathrm{D}-$ galactopyranosyl and $\mathrm{DA}$ unit $=3,6-$ anhydro- $\alpha-\mathrm{D}-$ galactopyranosyl) and differ on the number and position of the ester sulfate groups (S) [2]. There are three main commercial classes of carrageenans bearing one, two and three sulfate groups per disaccharide respectively: kappa (к: G4S-DA), iota (1: G4S-DA2S) and lambda ( $\lambda$ : G2S-D2S,6S)-carrageenans (Fig. 1). The best solubility into water is reached with the highest level of sulfate groups on the backbone: $\lambda$ carrageenans are soluble at all temperatures whereas $\kappa$ and $\mathrm{l}$ carrageenans, their potassium salts are soluble over $60-\mathrm{C}$ and their sodium salts are soluble at room temperature. Several chemical modifications of their free hydroxyl groups have been applied to carrageenans in order to modulate their physicochemical properties.

A last example of abundant and commercially available natural carbohydrate polymer extracted from red algae is agar-agar or simply agar. Accumulated in the cell wall of agarophytes, agar is a complex polysaccharide made up with a mixture of predominant neutral linear agarose and charged heterogeneous agaropectin with various kinds of substituents. The structure is a mixture of water-soluble galactans derivatives similar to carrageenan. The principal difference between agar and carrageenan is that (1-4) linked residues in agar are a L-enantioner, whereas in carrageenans, it is a D-enantiomer. As shown in Fig. 1, agarobiose is thus a disaccharide with a repeating unit $\rightarrow 3)-\beta$-D-Gal $p$ - $(1 \rightarrow 4)-3,6$-anhydro- $\alpha$-L-Gal $p$ - $(1 \rightarrow$. 3,6-anhydro-L-galactopyranosyl group is mainly responsible for the gelation properties and gel strength. The chemical nature of isolated agar is influenced by various factors as described for alginates.

This review will focus on the chemical modifications of the reactive functional groups carried on the backbone of the carbohydrate polymers. Examples applied to the four marine polysaccharides previously mentioned will 
be described. Two kinds of modifications will be discussed in detail such as substitution reactions and chain elongation via graft-copolymerization, cross-linking, polymer network and hydrogel formation. However, the very useful depolymerization reaction (chemical, physical or enzymatic) in order to obtain low-molecular weight (LMW) polysaccharides will not be broach here. Furthermore, it is noteworthy that we examined economically and industrially relevant polysaccharides that are the most explored and coming from sustainable sources.

The general chemical modifications outlined can be applied to other carbohydrates if the same reactive functional groups are carried on their backbone. As an example, fucoidans are also important sulfated starting material from the sea widely present among the brown algae. Only few structures have been established and they are often complex, heterogeneous and diverse [3]. In agreement with IUPAC recommendations, we stressed that sulfated fucans are polysaccharides based mainly on sulfated L-fucose with less than $10 \%$ of other monosaccharides. This term was applied to the sulfated fucans found in marine invertebrates, whereas the term fucoidan has been used for fucans extracted from algae [4]. Sulfate groups on the C2 or C4 of the monosaccharide may substitute the $\alpha$-L-fucopyranosyl residues.

Lastly, ulvan extracted from green seaweeds (Chlorophyta) is also a very attracted sulfated biopolymer from renewable resources. Ulvan polysaccharides showed complex heterogeneous chemical composition, which varies depending on several factors including the taxonomic and ecophysiological origins of the algal sources $[5]$.

Chemical modifications are a very efficient tool to improve the inherent properties of a molecule. Nevertheless, the modification of carbohydrate polymers presents some difficulties that have to be overcome such as $i$ ) complicated reaction conditions in order to make the most homogeneous medium, ii) low selectivity because of the high number of the same reactive groups along the backbone, iii) various side reactions difficult to avoid and iv) low yield of the expected final compound after purification techniques. These difficulties are mainly due to the heterogeneity of this kind of biomolecules, composed often of a mixture of polysaccharide chains. 


\section{CHEMICAL MODIFICATIONS BY SUBSTITUTION REACTION}

Efficient modification reactions have been developed in order to obtain well-defined advanced functional derivatives. Derivatization by introducing either small functional groups or new molecules of interest for targeted application can really improve physico-chemical properties of the starting polysaccharide. Several reviews describe chemical modifications of chitin/chitosan [6] and alginates [7]. Carrageenans can also be chemically modified but the literature is less abundant for this polysaccharide [8].

\subsection{Carboxyalkylation of chitin/chitosan}

The process of carboxyalkylation introduces acidic groups on the polymer backbone. The resulting derivative bears additional carboxyl groups as potential anionic charge with solubility depending on $\mathrm{pH}$.

Amino groups and hydroxyl groups are very reactive functionalities and the $O$ - versus $N$-selectivity has to be discussed using different reaction conditions.

Carboxymethylation of chitin and chitosan is a suitable way to overcome the limited solubility of the native polysaccharide. The hydrophobic methyl group disrupts the tough hydrogen bonds into the crystalline structure and the carboxyl group also enhances the solubility [9]. The resulting product is a glucan carrying pendant glycine groups. It is noteworthy that the reaction conditions applied could induce side reactions such as $N$ deacetylation, furnishing amphoteric compounds having both free carboxyl and amino groups or/and depolymerization of the native backbone of the carbohydrate polymer [10].

Starting with the chitosan activation by soaking it into a sodium hydroxide solution, the first method of direct alkylation induces the preparation of carboxymethyl-derivatives with monochloroacetic acid. The applied reactions conditions allow the $N$-versus $O$ - selectivity of the substitution (Fig. 2):

- the reaction medium is important because the solvent is a mixture of water and isopropanol with optimal ratio of $1: 1$ and 1:4;

- a mildly alkaline medium (pH 8-8.5) induces only amino group activation and yields mono and di- $N$ substituted compounds; while higher alkali conditions afford a final product as a mixture of $\mathrm{N}$ - and $O$-alkyl derivatives. The reaction proceeds at the $\mathrm{C}$ positions of the hexose preferably in the order $\mathrm{OH}(\mathrm{C} 6)>\mathrm{OH}(\mathrm{C} 3)>\mathrm{NH}_{2}(\mathrm{C} 2)$ yielding a respective amount of each product of $0.7>0.47>0.2$. In fact, a $\mathrm{NaOH}$ solution at $50 \%$ seems to provide the best conditions in the carboxymethylation process. 
At lower concentration, the tough crystalline organization of chitosan makes difficult the entrance of the chloroacetic acid into the polymer chains lowering the final degree of substitution (DS);

- $\quad$ the ratio of amine/acid determines the selectivity and the degree of substitution (1:1 for low DS to 1:4 for high DS).

To resume, direct alkylation reaction employs mild alkaline $\mathrm{pH}$ as well as inexpensive reagents and allows easy scale-up process.
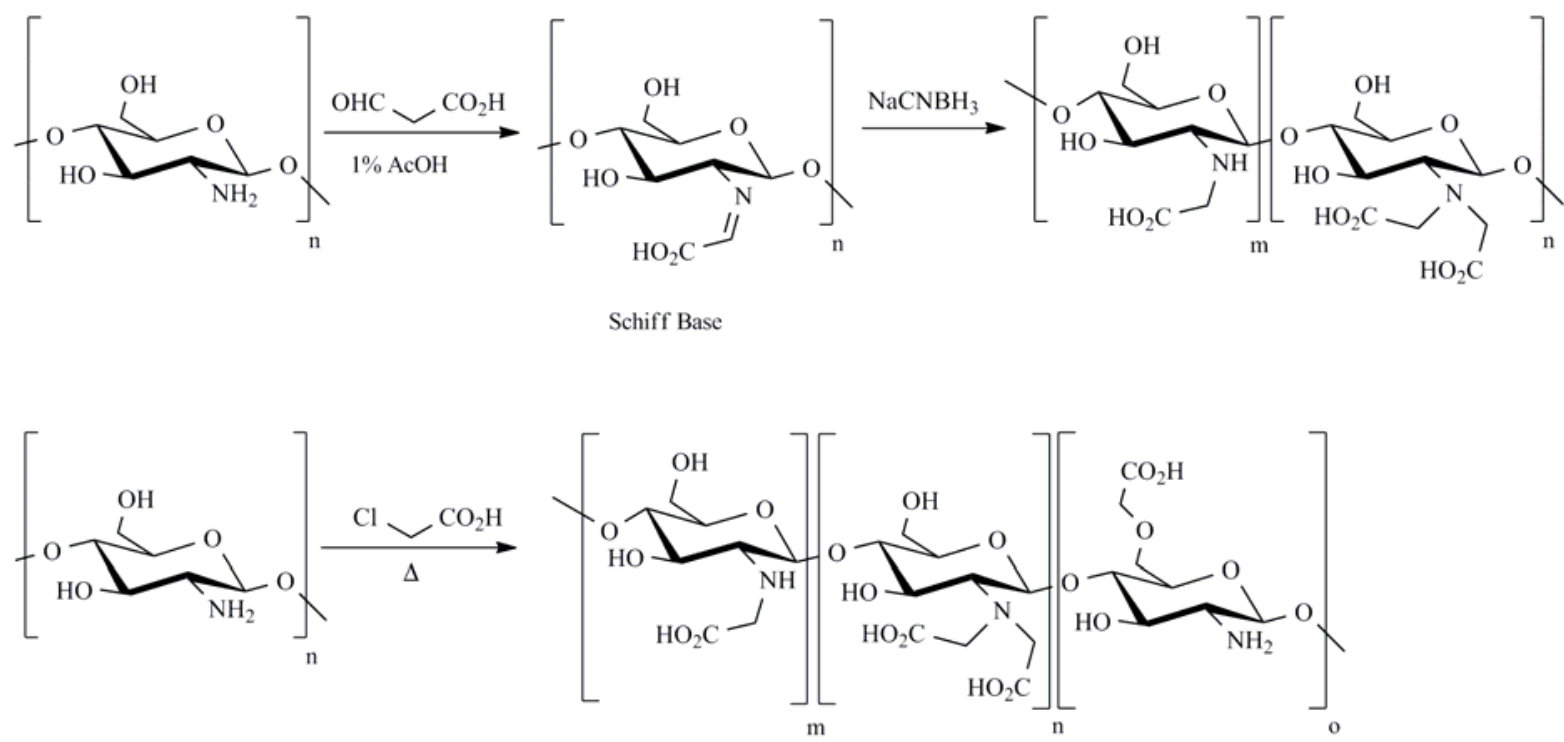

Fig. 2 : carboxyalkylation methods of chitosan

However, $\mathrm{N}$-carboxymethyl-chitosan is well defined and can be prepared in a very efficient way by using reductive amination. The first step is the Schiff base formation between amino group of chitosan and reagent bearing an aldehyde group (e.g. glyoxylic acid) or a ketone group. The resulting imine group is subsequently reduced (e.g. $\mathrm{NaBH}_{4}$ or $\mathrm{NaCNBH}_{3}$ ). Even so, partial production of $N, N$-dicarboxymethylated derivative cannot be avoided because of the high reactivity of the glyoxylic acid (Fig. 2). Furthermore, this method requires relatively expensive reagents and it is not easily applied to a larger scale.

This protocol could be extended with several other aliphatic or aromatic carboxylic acids providing higher homologs of the carboxymethyl-chitin or -chitosan.

Moreover, in comparison with the starting marine polysaccharide, these carboxyalkylated derivatives carry two reactive groups: remaining $\mathrm{NH}_{2}$ and new $\mathrm{CO}_{2} \mathrm{H}$. Additional modifications could be envisioned such as amide formation following classical methods of acylation, alkylation and more particularly quaternization of the amino group and even grafting (see next parts). 


\subsection{Acylation}

\subsubsection{Chitin/Chitosan}

A major limitation for chemical modification of marine polysaccharides is their poor solubility in organic solvent in order to undergo homogeneous reaction conditions. An obvious solution is to introduce hydrophobic moieties into the biopolymer structure to promote chemical reaction.

The smallest acyl group is the acetyl group, which can be introduced sluggishly onto chitin under heterogeneous conditions. Drastic conditions such as acetic anhydride and hydrogen chloride or methanesulfonic acid $\left(\mathrm{MeSO}_{3} \mathrm{H}\right)$ are necessary to fulfill extensive acetylation of chitin. These strongly acidic systems cause partial degradation of the polysaccharide backbone. Reactive $\beta$-chitin is not soluble in organic solvents but can swells in common solvent, i.e. methanol. Direct $N$-acetylation occurs in methanol thanks to acetic anhydride to give poly $(N$-acetyl-D-glucosamine $)$. Furthermore, $\beta$-chitin in pyridine is peracetylated with acetic anhydride as well but requires the presence of 4-dimethylaminopyridine (DMAP) used as catalyst in order to obtain a fully acetylated chitin. Scarce $\beta$-chitin is a more versatile starting material than the abundant $\alpha$-chitin. Acetylation of $\alpha$-chitin with structural uniformity to give a high degree of $O$-acetylation is more difficult; accordingly, more drastic conditions are required for its full acetylation. Taking advantage of solubilization properties of an ionic liquid, acetylation of $\alpha$-chitin was successfully performed in 1-allyl-3-methylimidazolium bromide [11].

As chitosan is a versatile molecule, various reactive functionalities along its chains can be involved during acylation. Reaction conditions can be selected to control the acylation reaction on an expected site, i.e. either amino, hydroxyl or both groups. Acylation proceeds preferentially at the free amino groups (C2) and then more slowly at the hydroxyl groups (C3 and C6). $N$-acyl derivatives of chitosan with hydrophobic side chains have a more soluble range than native chitosan. A wide variety of reagents yield chitosan derivatives with amide linkage owing to $N$-acylation reaction (Fig. 3):

- $\quad$ Acyl chloride or anhydride bearing various chains can be used, e.g. saturated or unsaturated acyl groups with different chain length as well as aromatic acyl groups;

- $\quad$ Less reactive carboxylic acids can react mediated by a coupling agent, e.g. hydrosoluble carbodiimide EDC (1-ethyl-3-(3-dimethylaminopropyl)carbodiimide hydrochloride), NHS $(N$ hydroxysuccinimide) or CDI ( $N-N^{\prime}$-carbonyldiimidazole), converting carboxylic acid group into an activated intermediate; 
- Cyclic acid anhydrides are successfully used giving $N$-carboxyacylated chitosan derivatives via ringopening reaction with terminal carboxyl group;

- Cyclic esters (lactones) yield derivatives with $N$-hydroxyalkanoyl groups with a terminal hydroxyl group.

It is noteworthy that terminal hydroxyl or carboxyl groups can further be chemically modified depending on the targeted properties.
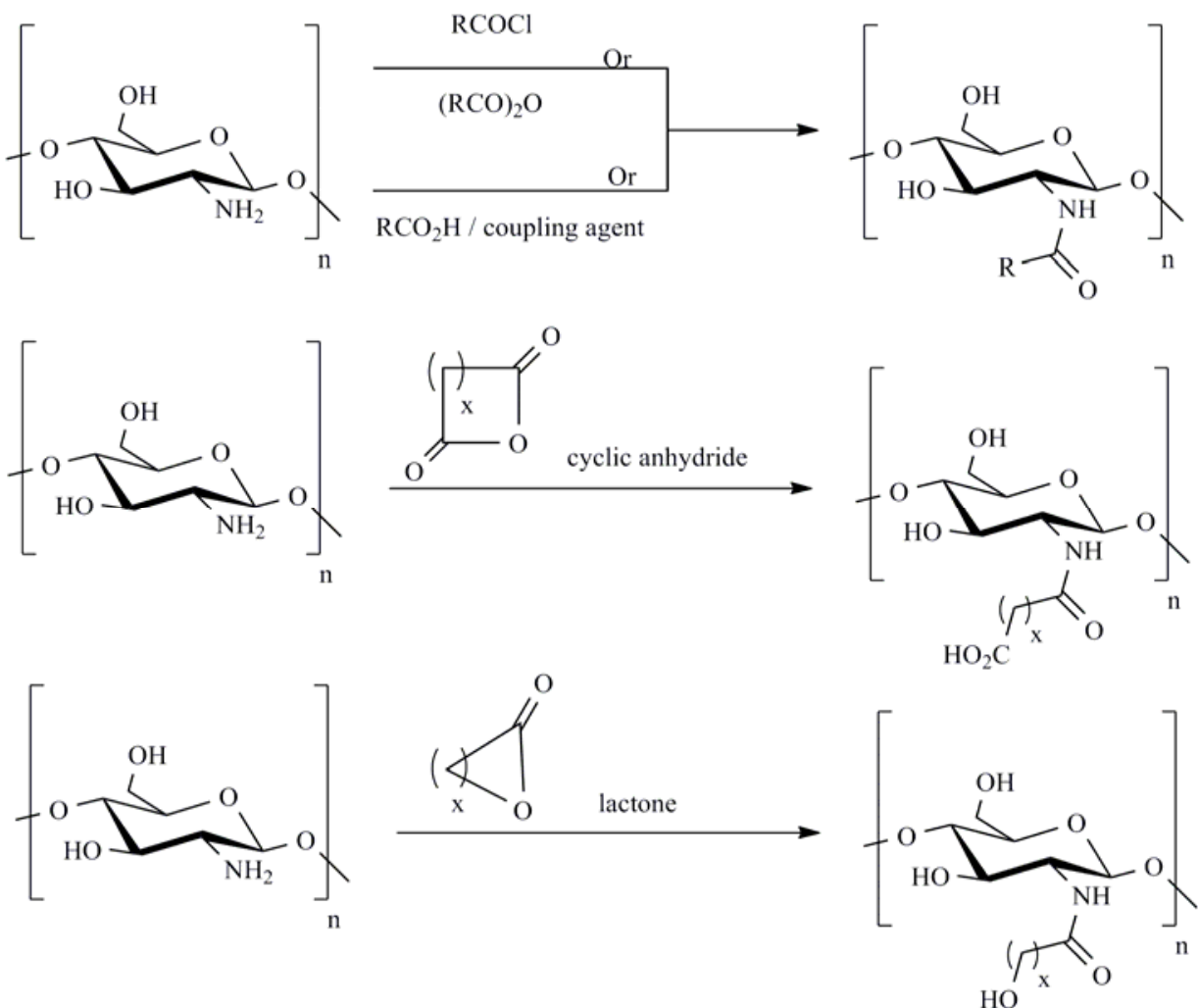

Fig. 3 : acylation of chitosan

$O$-acyl chitosan derivatives are interesting because of their ester linkage introduced by $O$-acylation reaction. Besides the contribution of a hydrophobic moiety, these molecules could be hydrolyzed by enzymes and are designed as biodegradable materials. $\mathrm{N}$ - $\mathrm{O}$-acyl chitosan was proposed in $\mathrm{MeSO}_{3} \mathrm{H}$ [12]. This solvent acts also as a protonating agent in order to form amino group salts. As a result, the substitution occurs preferentially on the hydroxyl groups. Since amino group is more reactive than the two hydroxyl groups, a protective group is often necessary to prepare $\mathrm{N}, \mathrm{O}$-acyl chitosan. In order to mask the amino group, $N$-phthaloyl-chitosan [13] derivatives are frequently chosen as intermediate. Thus, two additional steps of amino group protection and cleavage are necessary to induce chemoselectivity. One-pot synthesis for the $O$-acylation of chitosan has also been reported in $\mathrm{MeSO}_{3} \mathrm{H}[14]$. 
Native alginates are modified by direct esterification with alcohols in excess and in the presence of a catalyst.

The only derivative with a commercial value is the propylene glycol esters of alginate [15]. Butyl ester of alginate is prepared starting from alginate sodium salt through esterification with butanol catalyzed with concentrated sulfuric acid. The afforded material has kept the gelling and non-toxic properties of native alginates [16]. The use of carbodiimide coupling agent is also feasible. DMAP catalyst is added in order to obtain cholesteryl ester of alginate, which can self-assemble into more stable and compact nano-aggregates compared to native sodium alginate [17]. Esterification of the carboxylic acid group of alginates can occur via tetrabutylammonium salts to induce solubility in dimethylsulfoxide (DMSO) and reaction toward alkyl halide [18].

Preparation of alginate derivatives with amide linkage occurred between the carboxylate moiety of alginate backbone and an amine function. Classical peptide coupling agents, such as EDC, allow the amide function formation as additional hydrophobical part [19]. This process is not very efficient [20] and not completely regioselective because of numerous alcoholic functions on the polysaccharide backbone which may react as well and form esters groups.

\subsection{Oxidation}

\subsubsection{Additional carboxyl group}

A comment on the direct oxidation at $\mathrm{C} 6$ position of the chitosan is relevant in order to introduce a carboxylic acid function on the chitosan backbone. The 2,2,6,6-tetramethylpiperidine-1-oxy radical (TEMPO) $/ \mathrm{NaOCl} / \mathrm{NaBr}$ system oxidized the primary hydroxyl group of the chitosan after protection of the amine moieties in view of the characterization of the reaction products [21]. This system was previously used for chitin [22,23], $N$-acetylated chitosan [23] and chitosan [24].

Oxidized alginates have received much attention because they present more reactive groups, a faster degradation and are used as supports for drug controlled delivery [25]. On alginates, oxidation occurred at C2 and C3 position of the uronic acid units and are performed with sodium periodate. Two aldehyde groups are obtained in each oxidized monomeric unit [26]. 


\subsubsection{Chemical modifications of the carboxyl group}

\subsubsection{1. $\quad$ Esterification}

Introduction of ester groups on the polysaccharide backbone was explained in the previous acylation part.

\subsubsection{Multicomponent reactions}

The Passerini three-component condensation (Fig. 4) involves a carboxylic acid and a carbonyl (ketone or aldehyde) condensed with an isocyanide and after acyl rearrangement and tautomerization, finally yields $\alpha$ hydroxycarboxamide. Natural uronans or artificial ones, obtained by selective oxidation of primary alcohol group or carboxymethylation, are interesting because they can react in more regioselective reaction [27].

The Ugi four-component condensation (Fig. 4) between an aldehyde, an amine, a carboxylic acid and an isocyanide allows the preparation of $\alpha$-aminoacyl amide derivatives in one step. This reaction is useful to have access to peptidomimetics. The carboxylic acid function of alginates is involved in this multicomponent reaction. After $24 \mathrm{~h}$ under vigorous stirring, the reaction medium is formally dialyzed and then freeze-dried in order to obtain the final product [28].

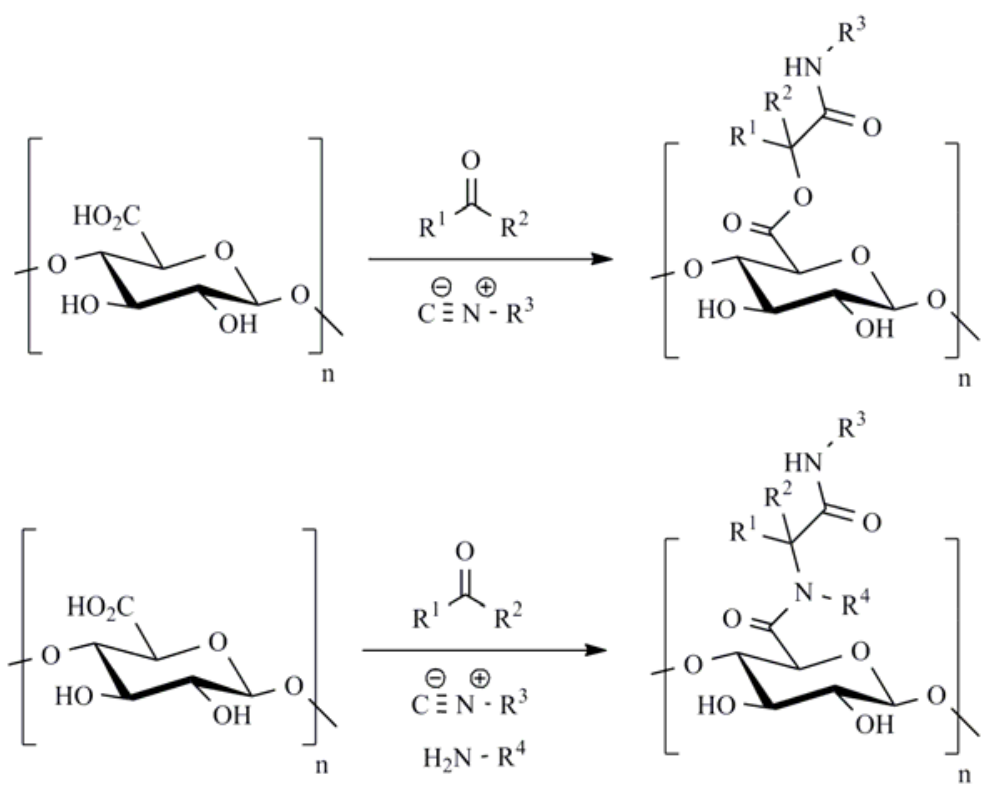

Fig. 4 : multicomponent reaction with chitosan

\subsubsection{Amidation}

Preparation of a polysaccharide with amide linkage occurred between a carboxylate moiety and an amine function. Various protocols were detailed in the previous acylation part. 


\subsection{Alkylation of chitin/chitosan}

Different procedures can be applied in order to introduce alkyl chains onto biopolymer backbone to confer hydrophobic nature (Fig. 5). The most reliable way of synthesis is to use reductive amination with Schiff base intermediate as previously described. Amino group undergoes reaction with aldehyde or ketone group furnishing an imine group, which could be consequently reduced to $N$ - alkyl chain upon hydrogenation with hydride. Another possibility is to use direct alkylation between the polysaccharide and an activated reagent such as an alkyl halide or an epoxide. The ratio $\mathrm{N}, \mathrm{O}$-substitution is thus determined by reaction conditions.
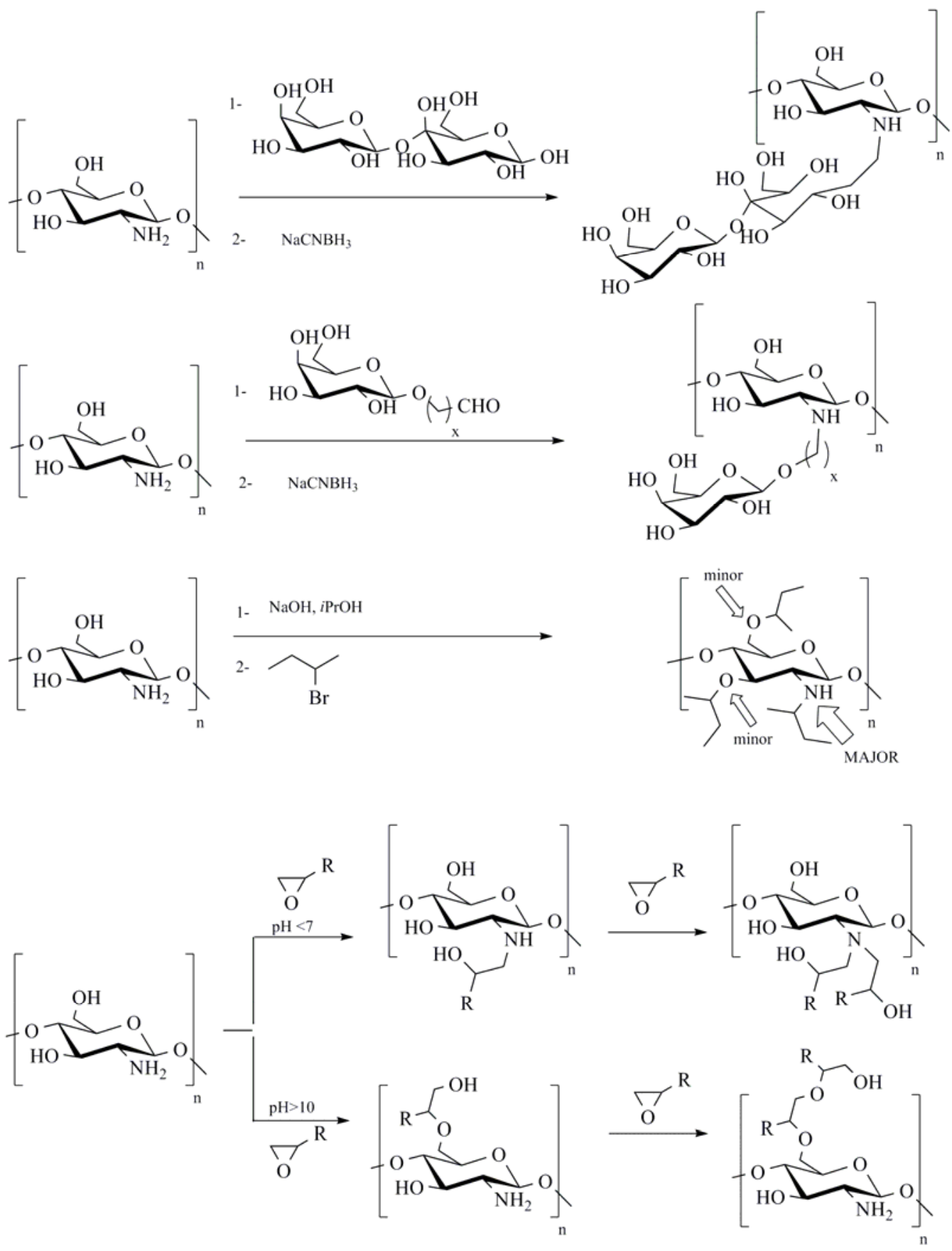

Fig. 5 : alkylations and hydroxyalkylation with chitosan 
The free primary amino group of chitosan undergoes Schiff base formation with aldehyde or ketone groups and a subsequent reduction provides the corresponding alkyl chitosan derivative, which could eventually be further quaternized on the amine group. Reductive $N$-alkylation of chitosan can be conducted with various aldehydes and saccharides [29,30]. Regarding reducing sugars, reductive amination proceeds with the aldehyde group of the open-chain form.

In order to keep all glycosidic linkage, reductive amination can also be applied to sugars bearing an aglycone part as a spacer with reactive aldehyde group. Consequently, the sugar part is incorporated without opening the sugar ring. Since the first reports of this approach [31], the new sugar introduced on the polysaccharide backbone could also contain other functional group, which do not react during the reductive $N$-alkylation and finally give new useful intermediate for further applications. For example, sialic acid is a prevalent sugar of the glycoproteins and glycolipids on the mammalian cell surface, which play a key role for a number of pathogenic infections. Sialic acid bound to chitosan was prepared via $p$-formylphenyl- $\alpha$-sialoside by reductive amination [32]. Since the obtained derivative was insoluble in water, subsequent $N$-succinylations were performed to afford a water-soluble derivative [32Erreur ! Signet non défini.,33].

Otherwise, alkylchitosan derivatives could be achieved through a direct alkylation reaction with alkyl halides under basic conditions. For example, the use of 2-bromobutane introduces an iso-butyl group on amino and hydroxyl groups of chitosan that enhances the solubility of the biopolymer in neutral aqueous solution [34]. This improved solubility could be attributed to the reduction of crystallinity of chitosan derivative. Reaction conditions (e.g. reaction time, temperature, and concentration of reactants) affect the degree of deacetylation, degree of substitution, viscosity-average molecular weight and viscosity of the final isobutyl chitosan derivative. Authors have underlined that isobutylchitosan presented chain degradation during alkalization and alkylation procedures. However, this alkylchitosan has a better biodegradability and the same non-cytotoxicity compared to chitosan.

Hydroxyalkylation process produces derivatives bearing an additional hydroxyl group. Direct alkylation is carried out via epoxide-ring opening occurring at the less substituted carbon under basic conditions or via substituted alkyl halide. Depending on reaction conditions, hydroxyalkylation may take place preferentially at amino function or hydroxyl group, yielding $N$-hydroxyalkyl or $O$-hydroxyalkyl derivatives or a mixture of both [35]. 2-hydroxyethyl derivative (or glycol derivative) is achieved thanks to ethylene oxide [6a] or 2chloroethanol [36] in alkaline medium. The reaction of chitin with ethylene oxide would induce side reactions such as deacetylation under strongly basic conditions or polymerization introducing oligo(ethylene glycol) side 
chains. These side reactions lead to structurally ambiguous derivatives. Furthermore, two hydroxyl groups could be introduced in one step using glycidol or 3-chloropropane-1,2-diol [37].

The increase of the cationic character of chitosan has many advantages and offers a broad range of applications [6g]. Subsequent methylation of the amino group of chitosan can be achieved with methyl iodide in alkaline solution of $N$-methyl pyrrolidinone (NMP) [38]. $N, N, N$-trimethyl chitosan chloride was obtained after ionexchange with sodium chloride (Fig. 6). These reaction conditions lead to polymer chain scission [39], partial and uncontrolled $O$-methylation [40] and the degree of quaternization [41] is difficult to control. Another two steps method is described to synthetize the expected $N, N, N$-trimethyl chitosan derivative with tailorable degree of quaternization and avoids $O$-methylation and chain scission as side reaction [42]. It is noteworthy that $O$ methylation decreases the quaternized chitosan solubility [40a]. Another method was also proposed using dimethylsulfate as active reagent without solvent [43].
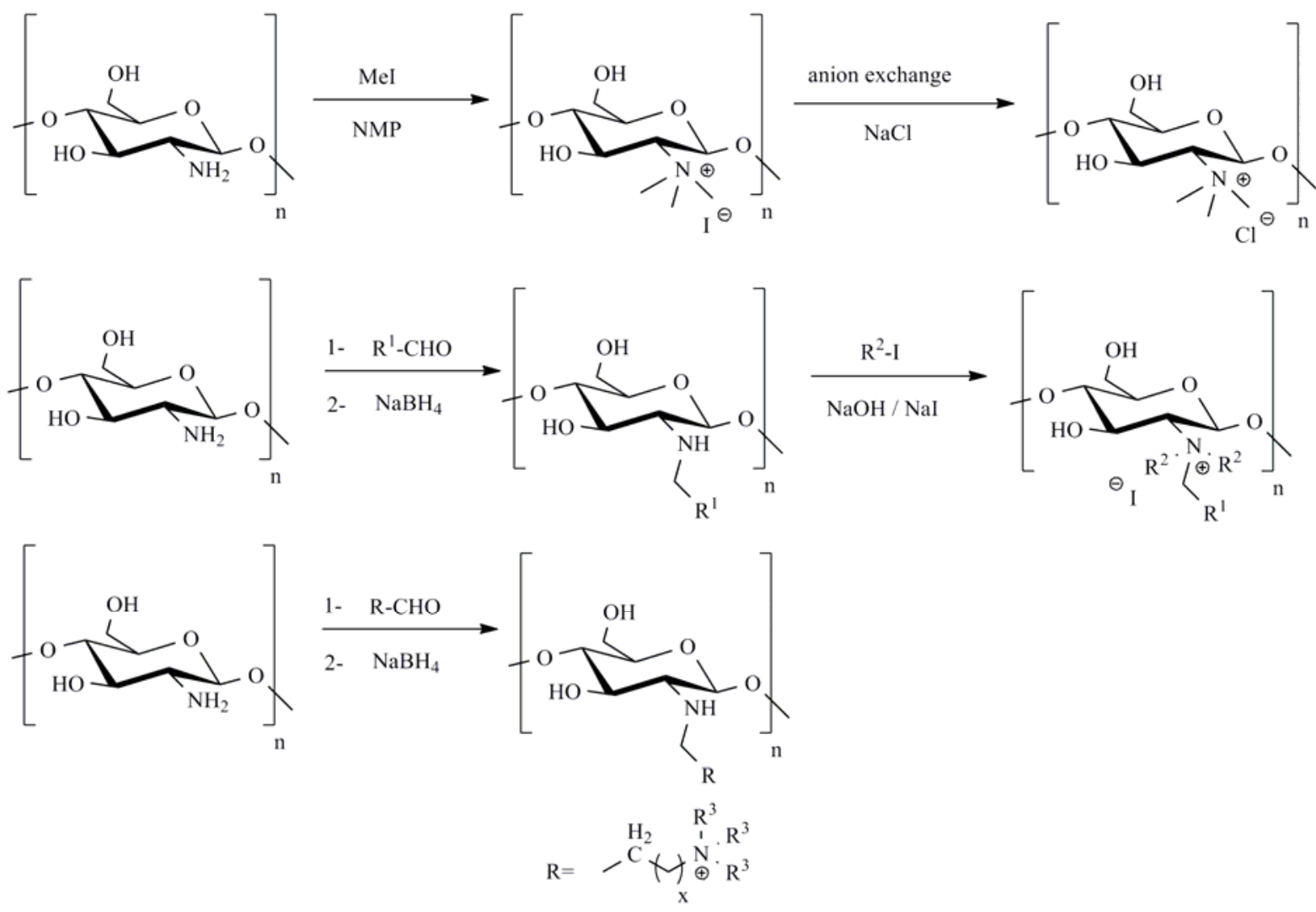

Fig. 6 : quaternization of chitosan

Other quaternary ammonium chitosan derivatives can be prepared. $N$-alkyl derivatives obtained via reductive alkylation can be subjected to subsequent quaternization [44]. The ammonium part could also be directly 
introduced as side chain on the biopolymer backbone via $\mathrm{N}$-hydroxyalkylation with quaternized epoxides such as glycidyltrimethylammonium chloride [45] or $O$-alkylation [46].

\subsection{Sulfation}

Chemical modification of biopolymer is often performed to generate new biofunctional materials without modifying the fundamental backbone and with the purpose of keeping the original physicochemical and biochemical properties.

Focusing on medicinal applications, sulfated polysaccharides are very attractive bioactive products. For example, heparin is the anticoagulant and antithrombotic agent the most commonly used in the prevention and treatment of venous thrombosis [47]. This essential commercially available drug is extracted from mucous lining of pig intestines. Because of its animal origin, the heparin can be potentially contaminated with pathogenic agents or chemical contaminants. The presence of chemical contaminants such as an unusual tetrasulfated disaccharide repeating unit has been reported, they can provoke fatal anaphylactic responses [48,49]. As a consequence, marine bacterial polysaccharides are a promising response to reduce, indeed avoid, the risk of contamination with mammalian pathogenic agents.

Chemical sulfation is an efficient tool to obtain semi-synthetic heparin-like drugs from non-mammalian polysaccharides complying with a glycosaminoglycan (GAG) strategy [50].

For improving the production of GAG-like derivatives, one-step chemical sulfation of polysaccharides is a challenge because this step presents several limitations: $(i)$ bringing together hydrophilic natural macromolecules and organic reagents in homogeneous conditions, (ii) difficulties for driving the reaction to completion due to anionic crowing which increases with the number of linked sulfate groups, (iii) lack of control and reproducibility toward the reaction (e.g. regioselectivity), (iv) lability of sulfate groups in acidic conditions or with high temperatures and $(v)$ difficulty to remove high proportion of inorganic salts at the end of the reaction[51]. Considering that sulfate groups are sensitive and that highly sulfated products have valuable biological targets, sulfation reaction is mostly carried out as final chemical modification of the polysaccharide.

The biological activity of polysaccharide depends on several structural parameters such as the degree of sulfation (DS), the molecular weight, the sulfation position, type of sugar, and glycosidic branching [52]. 


\subsubsection{Chitin/Chitosan}

One of the major interests about sulfated chitin/chitosan is the application as analogue of the natural blood anticoagulant heparin but also as novel biomaterials [53].

Sulfation reaction carried out on multi-functional polysaccharides, such as chitin or chitosan, would insert structural heterogeneity in polymer chains with random $\mathrm{N}, \mathrm{O}$-sulfation (Fig. 7). This phenomenon is increased because of the heterogeneous medium of the conventional sulfation procedure [54]. It is very difficult to prepare sulfate derivatives with high degree of substitution values [55]. Harsh reaction conditions applied can lead to severe molecular degradation and other side reactions resulted in products with dark color [56,36a].

Chitin and chitosan sulfate esters have been prepared using various sulfation reagents including concentrated sulfuric acid [57], oleum [58], chlorosulfonic acid [59], sulfur trioxide [60], sulfur trioxide complex (e.g. with pyridine [61], trimethylamine [62]). After chemical modification, the ability of forming hydrogen bond was decreased. The thermal stability of $N$-alkyl- $O$-sulfated chitosan was less than the starting chitosan. As a consequence, introduction of substituents into polysaccharide should disrupt the crystalline structure [59].

Sulfation reaction are performed in usual solvents [63] such as formamide, $N, N^{\prime}$-dimethylformamide (DMF), DMSO or pyridine and carried out on low-molecular weight polysaccharide [64].

In order to obtain well-defined sulfated-chitosan derivatives, selective $O$-sulfation could occurs starting from $\mathrm{N}$ acyl or $\mathrm{N}$-alkyl chitosan. Moreover, an additional trityl protective group on the primary hydroxyl group on $\mathrm{C} 6$ position of the chitosan could allow sulfation on remaining reactive positions or permit modification on other positions and keep hydroxyl group on C6 position after deprotection [65].

It was also reported that the sulfate group could be introduced thanks to a sulfated side chain (e.g. 5-formyl-2furansulfonic acid, sodium salt) using the mild conditions of the Schiff reaction to avoid polymer degradation and $O$-alkylation [66]. 


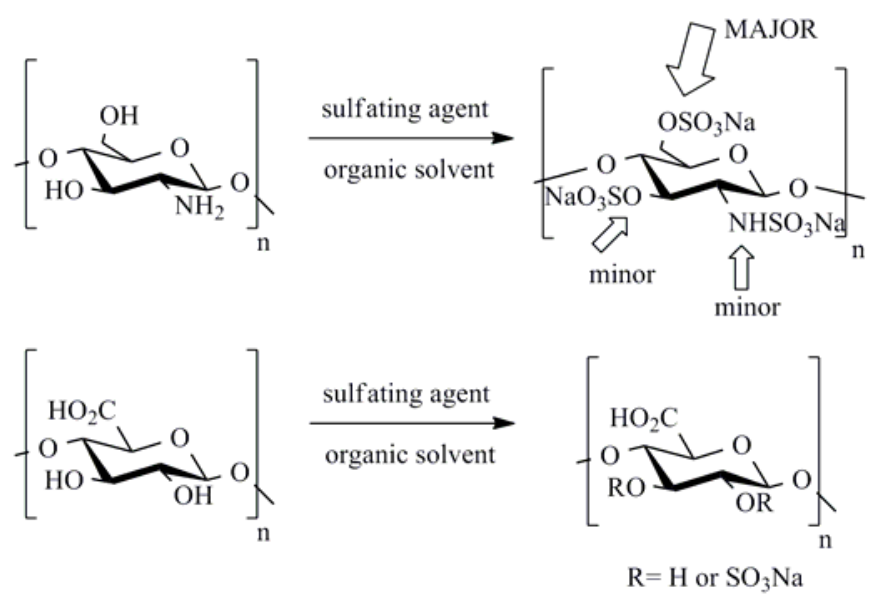

Fig. 7 : sulfation of chitosan or alginate

\subsubsection{Alginates}

Sulfated alginates show a high blood compatibility and anticoagulant activity because of its heparin-like structure [67]. In addition to classical sulfation reaction achieved with chlorosulfonic acid in formamide [68], carbodiimide coupling chemistry [69] or unusual sodium bisulfite and sodium nitrite [70] were also used. Sulfation sequence and pattern have not been yet controlled and identified precisely (Fig. 7).

\subsection{Phosphorous-containing derivatives}

\subsubsection{Chitin/Chitosan}

Several techniques to obtain phosphorus-containing derivatives have been proposed (Fig. 8). Bactericidal, biocompatible, bioabsorbable, osteoinductive and metal chelating properties justify the interest on phosphoruscontaining chitin/chitosan [71].

Phosphorylation of hydroxyl groups of chitosan to give phosphonate has been studied according to two main ways: $i)$ phosphorus pentoxide $\left(\mathrm{P}_{2} \mathrm{O}_{5}\right)$ - methane sulfonic acid system [72], ii) phosphoric acid $\left(\mathrm{H}_{3} \mathrm{PO}_{4}\right)$ in the presence of urea [73]. Water-soluble $N$-mono and $N$-di-phosphonicmethylene chitosan have been prepared with phosphorous acid $\left(\mathrm{H}_{3} \mathrm{PO}_{3}\right)$ and formaldehyde sequentially or simultaneously in aqueous acidic medium [74]. Namely, a three-component coupling of a carbonyl (e.g. formaldehyde), an amine (e.g. amino group of the chitosan) and a hydrophosphoryl compound leads to $\alpha$-aminophosphonate following Kabachnik-Fields reaction. Phosphorylated chitin [75] and chitosan [76] can also be prepared using $\mathrm{H}_{3} \mathrm{PO}_{4} / \mathrm{Et}_{3} \mathrm{PO}_{4} / \mathrm{P}_{2} \mathrm{O}_{5} /$ hexanol method. 

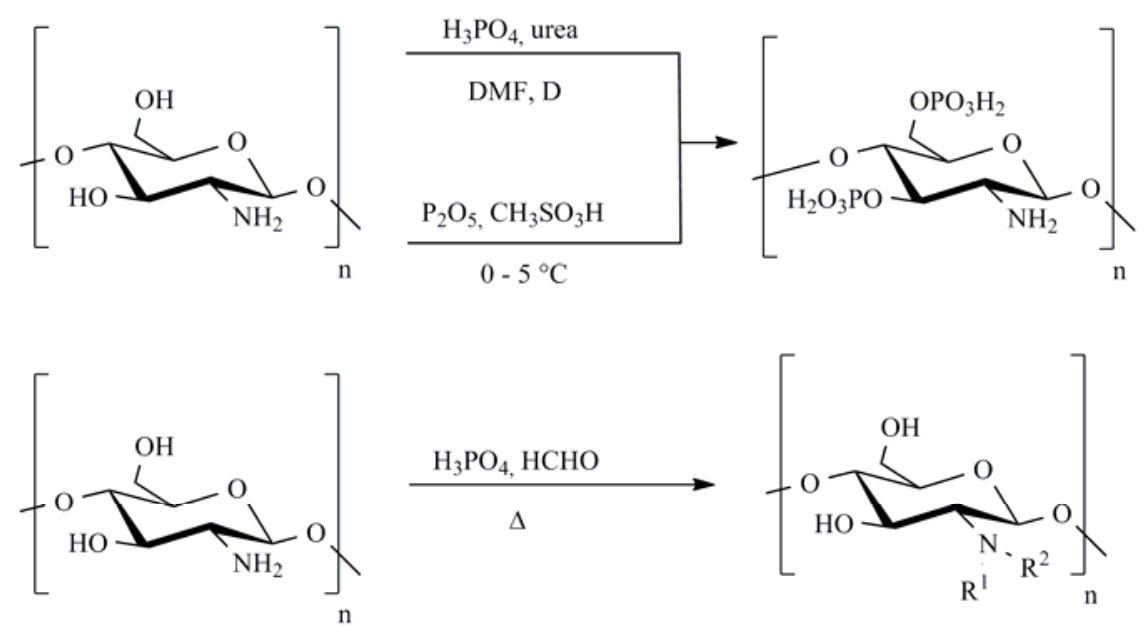

$$
\begin{aligned}
& \mathrm{R}^{1}=\mathrm{H}, \mathrm{R}^{2}=\mathrm{CH}_{2} \mathrm{PO}_{3} \mathrm{H}_{2} \\
& \text { or } \mathrm{R}^{1}=\mathrm{R}^{2}=\mathrm{CH}_{2} \mathrm{PO}_{3} \mathrm{H}_{2}
\end{aligned}
$$
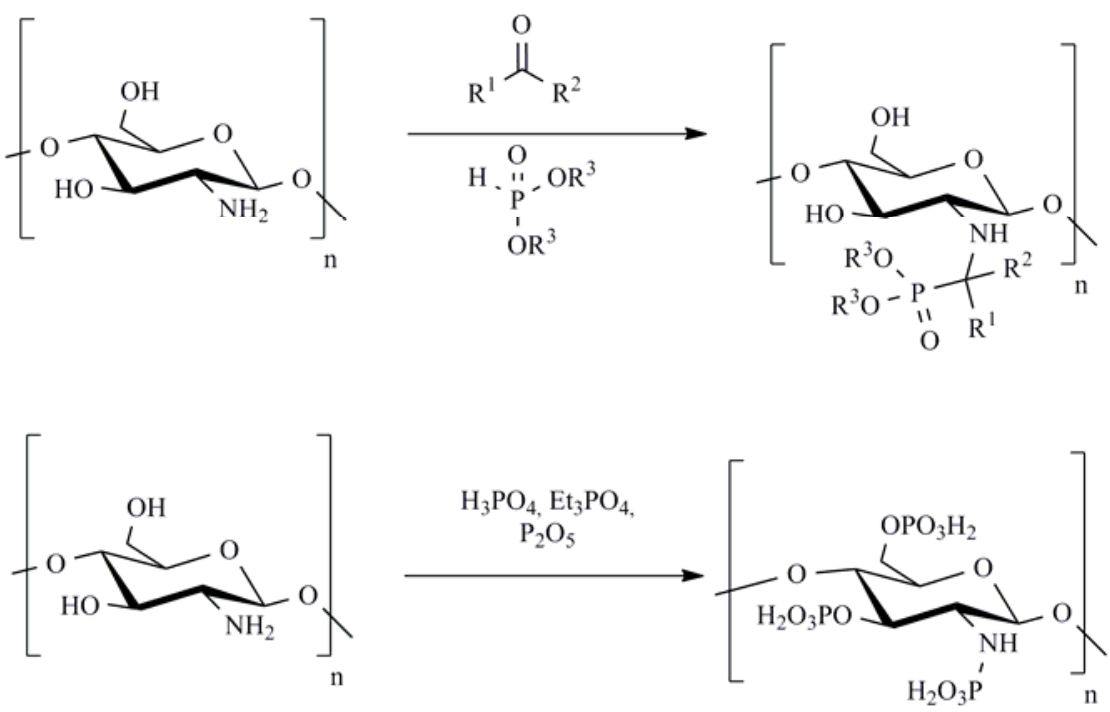

Fig. 8 : phosphorylated chitosan

\subsubsection{Alginates}

Phosphorylated alginate derivatives were described to evaluate their ability to induce hydroxyapatite nucleation and growth [77]. Phosphorylation of alginate was achieved using a heterogeneous urea/phosphate reaction. Regioselectivity of phosphorylation reaction was studied using NMR spectroscopy. It is noteworthy to highlight that the high acidity of the reaction significantly decreases the molecular weight of the resulting phosphorylated alginate derivative obtained. As a result, it diminished the ability of the resulting polysaccharide to form calcium cross-linked hydrogels. Blending unreacted alginate with phosphorylated alginate derivatives could form calcium cross-linked gels. Such gels showed higher resistance to calcium extraction compared to gel formation using only unreacted alginates. 


\subsection{Thiolation of chitin/chitosan}

Widely investigated for non-invasive drug delivery, thiomers are thiolated polymers which backbone bears thiol (or sulfhydryl) group ligands. Unexpected oxidation of the thiol group during synthesis can be avoided by performing the reaction under inert conditions. Alternatively, reaction can be conducted at $\mathrm{pH}$ below 5 inducing a low concentration of thiolate anions and the disulfide bonds can be excluded. Thiomers remain sensitive and reactive molecules $[6 \mathrm{~g}]$. The amount of thiol groups remaining on the polymer can be finally determined by Ellman's method [78].

Derivatization of the primary amino group of chitosan with reagent displaying thiol functionality provides thiolated chitosan derivatives. Mucoadhesive, permeation enhancing, in situ gelling, efflux pump inhibitory, controlled drug release properties and biodegradability are improved [79]. Various techniques have been applied in order to obtain these thio-derivatives (Fig. 9).
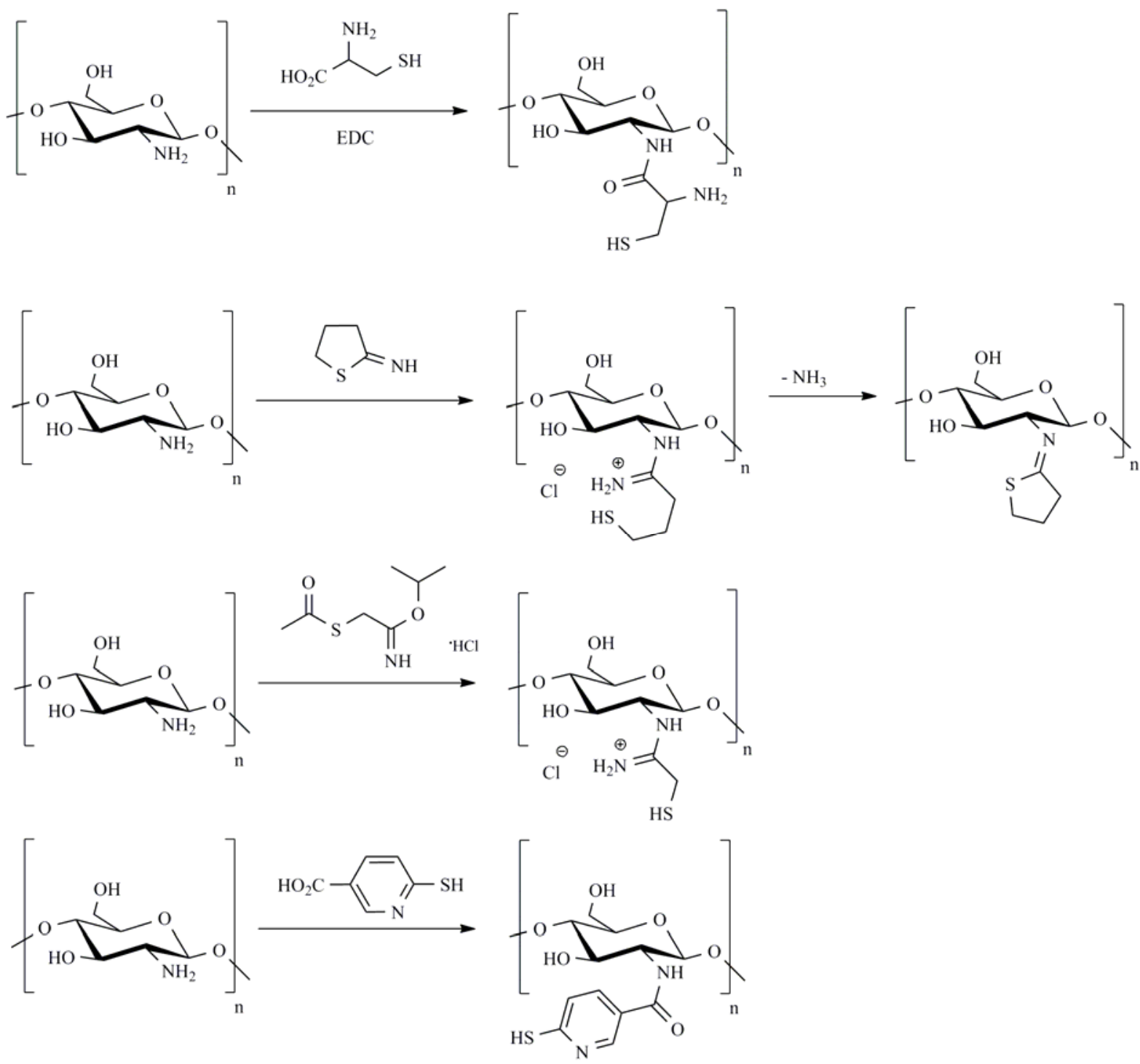

Fig. 9 : thiolation of chitosan 
$N$-acylation previously mentioned is a typical procedure to obtain thiol-bearing compounds [80]. Via coupling reagent (e.g. carbodiimide), designed activated carboxylic acid (sulfhydryl-bearing compounds [81] such as cysteine or thioglycolic acid) can form amide bond. 2-iminothiolane reagent (i.e. a cyclic thioimidester or thioimidate) can react with chitosan in order to provide positively charged conjugate with amidine bond. For example, chitosan-4-thiobutyl-amidine is obtained but shows an insufficient stability event stored under nitrogen [82]. In order to achieve the same properties of the previous chitosan derivative, isopropyl- $S$-acetyl thioacetimidate hydrochloride was used to give resulting chitosan-thioethylamidine derivative [83]. Arylthiolbearing molecules linked to chitosan thanks to amide bond can also be obtained [84].

\subsection{Regioselectivity}

As polysaccharides are complex macromolecules with several reactive groups, protective groups can be utilized in order to make chemical modification on a specific position. As a result, reliable key intermediates for regioselective chemical modifications are synthetized to provide stable derivatives that are structurally welldefined compounds. The transformations have to proceed smoothly in a quantitative term of the degree of substitution.

\subsubsection{Amino group at $\mathrm{C} 2$ position : $N$-Phthaloylation}

$\mathrm{N}$-phthaloylation is the most common technique for protection of the amino group of the chitosan as well as for enhancement of the solubility of the polysaccharide in common organic solvents. In order to avoid inherent mix of $\mathrm{N}$-O-phthaloylation on chitosan and subsequent removing of $O$-phthaloyl groups, phthaloylation can be realized in the presence of some water. A fully $2-N$-phthaloylchitosan is obtained which is a convenient intermediate to introduce first substituents at the C6 position and then regenerates the initial amine group thanks to final hydrazinolysis step [13]. Even if the $N$-phthaloylation is a well-known procedure, some reports noticed a significant decrease in molecular weights with the applied amino group protection-deprotection strategy [85]. The polysaccharide backbone degradation has to be taken into consideration when preparing derivatives for different applications. 


\subsubsection{Primary hydroxyl group at $\mathrm{C6}$ position: $O$-Tritylation, $O$-Tosylation, $O$ - Trimethylsilylation}

Preparing advanced functional materials is essential to perform reactions in a well-controlled manner. Thus, bulky protective groups could be introduced at the less hindered position (e.g. C6 hydroxyl group). Starting from $N$-phthaloylchitosan [86], triphenylmethylation (tritylation, Tr) or p-toluenesulfonylation (tosylation, Ts) can be conducted. More than a real protective group, tosyl group is considered as good leaving group for the resulting activated C6 position. Therefore, $O$-tosyl group can be converted into iodo, methyl or thiol group [87]. $O$ trimethylsilylchitosan is attractive because it enhances the polysaccharide solubility and eases further chemical modifications [88].

\subsection{Click Chemistry}

K. B. Sharpless coined the catchy term "click-chemistry" [89] and described reactions which are regiospecific, highly efficient, modular, wide in scope, generating no or harmless by-products, involving readily available starting materials and reagents. Moreover, this reaction can be performed under simple mild conditions in easily removable or benign solvents in order to obtain a product requiring no or minimal purification. As a consequence, several chemical reactions can fulfill these criteria. We will focus here on common click reactions employed in polymer science and more precisely cycloaddition reactions and thiol-ene couplings.

Development of click-chemistry is a powerful tool in the synthesis of tailored polymers for biomedical and pharmaceutical applications and their importance increases in the recent literature. Click-chemistry could overcome difficulties to obtain chemically modified polysaccharides, e.g. low selectivity, side reactions, low yields and low substitution efficiency. The impact of click-chemistry on carbohydrate chemistry, and more precisely polysaccharide chemistry, has contributed to extensive application to generate complex glycoconjugates with the postfunctionalisation of polymers with carbohydrates [90].

The purpose of this review is not to summarize all of the work to date on the application of click-chemistry in polymer science but to focus on marine polysaccharides. Useful chemical modifications on targeted groups of these biopolymers backbone would be highlighted implementing this powerful ligation technique.

\subsubsection{Triazole linkage}

Two independent research groups [91] reported the real improvement of $\mathrm{Cu}(\mathrm{I})$ catalyst during Huisgen 1,3dipolar cycloaddition reaction between an organic azide and a terminal alkyne. The affording product is 
exclusively a 1,4-disubstituted-1,2,3-triazole at $10^{7}$ times faster rate than uncatalyzed thermal reaction. This coupling process relies on copper(I)-catalyzed azide-alkyne cycloaddition (CuAAC) and the $\mathrm{Cu}(\mathrm{I})$ source can be added directly into reaction, or it is in situ generated by reduction of $\mathrm{Cu}$ (II) salts or by oxidation of elemental copper. CuAAC stands out as a first example of click-chemistry as a valuable tool to modify complex scaffolds and biomolecules. Later, ruthenium-catalyzed 1,3-dipolar cycloaddition reaction (RuAAC) [92] was discovered, which produces exclusively 1,5-disubstituted-1,2,3-triazoles. This cycloaddition was poorly investigated because of its sensitivity to solvent and steric demands of azide substituents in comparison to CuAAC.

To explain the major interest of CuAAC reaction as powerful tool: copolymers can be prepared in one step and the obtained triazole ring represents bioisosteres of amide bond [90]. Chemical and electronic resemblances make this heterocycle biologically relevant. 1,4-disubstituted-1,2,3-triazoles prepared via CuAAC mimic Zamide bond and 1,5-disubstituted-1,2,3-triazoles are similar to $E$-amide bond.

Nevertheless, another kind of cycloaddition was investigated giving also triazoles: strain-promoted azide-alkyne coupling (SPAAC) [93]. This reaction is very attractive because it can avoid copper salt catalysis with its two major side effects on polysaccharides: depolymerization and contamination [94]. To react with an azide, a key intermediate has to be prepared: a cyclooctyne, which can be activated by electron-withdrawing groups. Unfortunately, difluorinated cyclooctynes are rather difficult to synthetize [95].

A last example of copper-free click reactions is a tandem [3+2] cycloaddition-retro-Diels-Alder ligation method in which trifluoromethyl-substituted oxanorbornadiene derivatives react with an azide to form a triazole linkage [96]. The advantage of these oxanorbornadiene derivatives is their easier synthesis in comparison to difluorinated cyclooctynes preparation. Generally, Diels-Alder reactions have been extensively considered for the synthesis of dendrimers, star-like polymers, graft-copolymers and other highly congested macromolecular structures [97].

An overview of copper-free azide-alkyne cycloadditions and their perspectives were underlined [98].

\subsubsection{Thiol-ene coupling reactions}

Hydrothiolation of any alkene bond can proceed by either anti-Markovnikov radical addition or base-catalyzed Michael addition. The radical mediated thiol-ene reaction was reported as "thio-click" in 2007 [99]. Thioether linkage is provided owing to Michael-type addition of thiols to electron-deficient alkenes (e.g. acrylates, acrylamides, vinyl sulfones, maleimides). The high nucleophilicity of the sulhydryl moiety allows the thiol-ene coupling reaction to proceed under physiological conditions. The significant drawbacks of thiol-ene reactions 
should be pointed out as the prevalent thiol odor and the sensibility of the thiol group towards oxidation, as discussed previously. Unwanted disulfide bond formation can be avoided by excluding oxygen from the reaction medium or by adding reducing agents or by using protected thiol group derivatives.

Radical addition of thiols to alkynes has been recently added to the click reactions toolbox for the facile preparation of multifunctional polymer structures [100].

\subsubsection{Examples of "clickable" derivatives}

As this review focuses on marine polysaccharides, click chemistry was applied essentially to chitosan derivatives generating triazole link starting from azido-derivatives (Fig. 10).
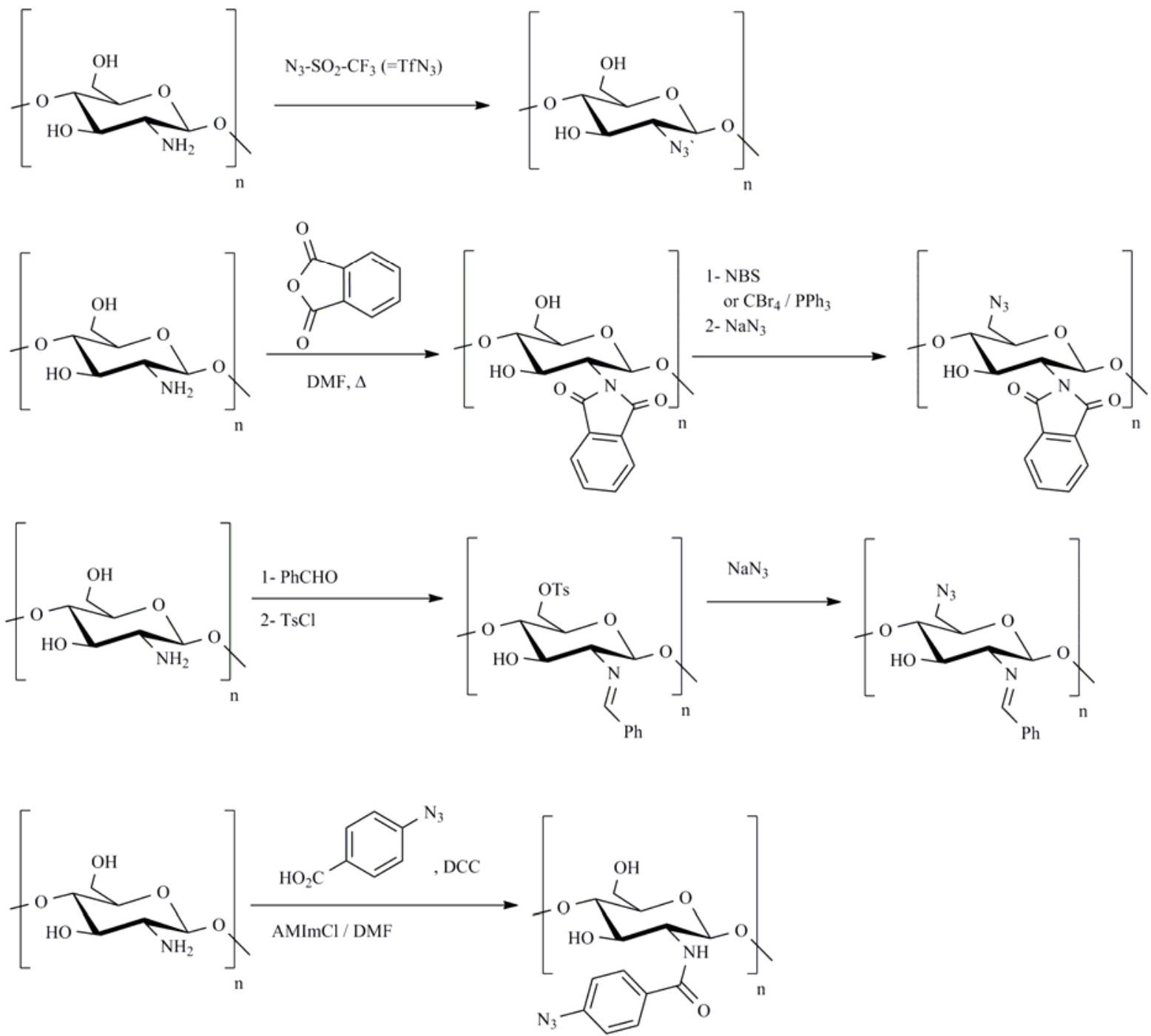

Fig. 10 : azido-chitosan 


\subsubsection{1. $\quad N$-azidation of chitosan}

The original pathway was established by an azido-functionalisation carried out using a $\mathrm{CuSO}_{4}$-catalyzed diazo transfer from trifluoromethanesulfonyl azide (triflyl azide, $\mathrm{TfN}_{3}$ ) or uncatalyzed diazo transfer of triflic anhydride $\left(\mathrm{Tf}_{2} \mathrm{O}\right)$ [101]. The reactions were performed until the Kaiser test [102] was negative, i.e. no free amino groups remained. A warning was mentioned that neat $\operatorname{TfN}_{3}$ has been reported to be explosive [103]. After comparison to sodium azide and sodium nitrite and imidazole-1-sulfonyl azide hydrochloride, the most convenient method of azidation is triflic azide [104]. Azido group could also be brought thanks to an azidolinker, which reacts with free amino group of chitosan [105]. The application of SPAAC is efficient for decoration of an azido-PEG linker on chitosan backbone to provide nanostructures with an IgG under physiological conditions [106].

\subsubsection{C6 azido functionalized chitosan}

Deoxyhalogenation (via bromide [107] or iodide [108]) of $N$-protected chitosan (e.g. phthaloyl [107] or Schiff base [109] protected) and subsequent azidation was described. Deoxybromination and azidation could also occur without isolation of the intermediate [110].

\subsubsection{Oxanorbornadiene approach}

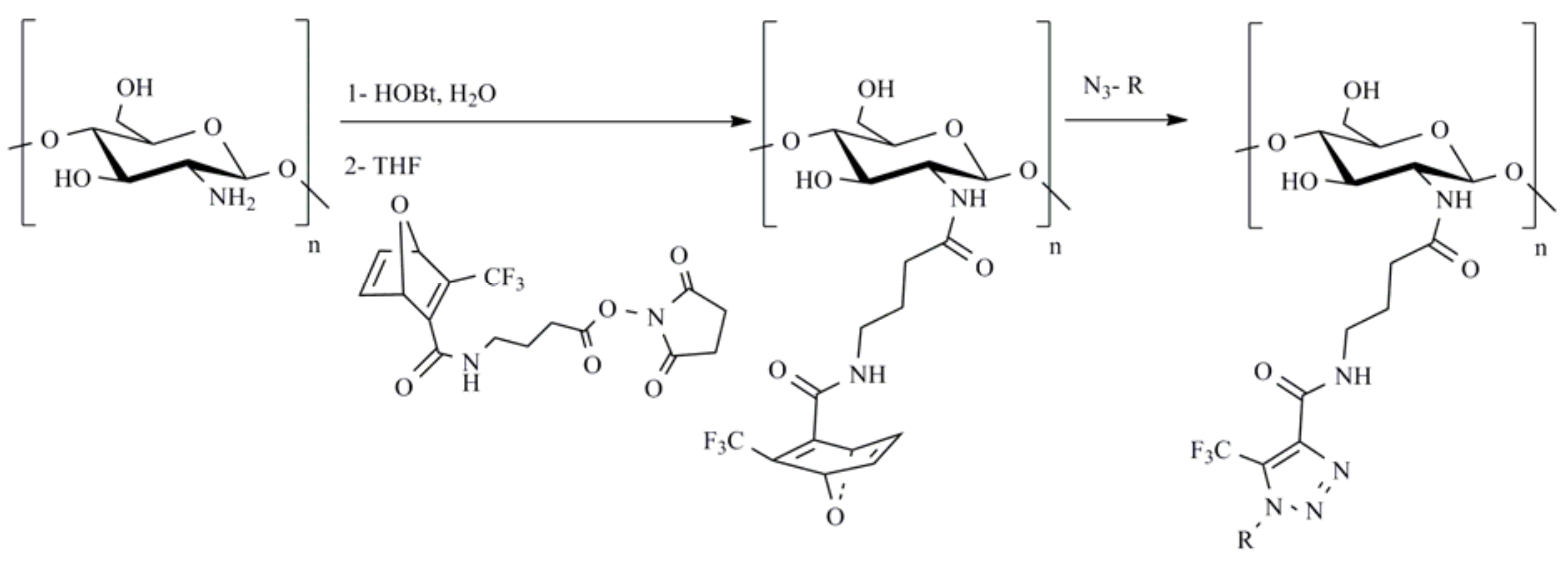

Fig. 11 : oxanorbornadiene strategy applied to chitosan

Introduction of trifluoromethyloxanorbornadiene moiety to chitosan was conducted via a spacer bearing NHSactive ester derivative (Fig. 11). A triazole linkage was successfully created by a cycloaddition reaction, which is considered as a simple ligation to other functional polymers or inorganic particles [111]. 
Rutjes' oxanorbornadiene approach was recently applied to functionalize alginates involved in metal-free cycloaddition reaction with azido-cyclic RGD pentapeptide for potential applications in regenerative medicine $[112]$.

\section{CHEMICAL MODIFICATION BY CHAIN ELONGATION}

Graft copolymerization is a promising technique to modify or introduce new physical and chemical properties via covalent linkages of synthetic polymer chains onto polysaccharides for producing novel derivatives useful in various fields. The resulting graft copolymers possess new properties mainly due to the characteristics of the synthetic side chains such as length, density or chemical structure. Researches in this field have blossomed quickly and are still an extremely active subject of study for various applications as in cosmetic, medicine, sensors or treatments of wastewaters.

\subsection{Graft copolymerization}

\subsubsection{Chitin / Chitosan}

\subsubsection{Grafting initiated by free radicals}

Graft copolymerization of vinylic monomers onto a trunk polymer of chitin or chitosan using free radical initiation has been explored widely in the last thirty years. In order to obtain tailored polysaccharide- $g$-polymer with a great variety of functionalities or different patterns, numerous initiator systems were successfully identified. The earliest redox systems reported in the literature that allow the grafting of vinyl monomers were based onto the Fenton's reagent $\left(\mathrm{Fe}^{2+} / \mathrm{H}_{2} \mathrm{O}_{2}\right)$, the cerium (IV) ion under acidic aqueous conditions, and tributyl borane (TBB) [113]. A modified Fenton's reagent with potassium persulfate (PPS) instead of hydrogen peroxide has also been reported [114].

To give a detailed mechanism as example, the Fenton's reagent is a reductive-oxidative reaction between the ferrous ions, which comes from ferrous ammonium sulfate (FAS) and hydrogen peroxide leading to the formation of hydroxyl radicals (Fig. 12) [113a]. Then, hydrogen abstractions due to hydroxyl radicals afford macroradicals along the polysaccharide trunk that initiate the growth of grafted synthetic chains from vinyl monomers. 


$$
\begin{aligned}
\mathrm{H}_{2} \mathrm{O}_{2}+\mathrm{Fe}^{2+} & \longrightarrow \mathrm{HO}^{+}+\mathrm{HO}^{-}+\mathrm{Fe}^{3+} \\
\mathrm{HO}+\mathrm{Fe}^{2+} & \longrightarrow \mathrm{HO}^{-}+\mathrm{Fe}^{3+} \\
\mathrm{HO}+\mathrm{H}_{2} \mathrm{O}_{2} & \longrightarrow \mathrm{H}_{2} \mathrm{O}+\mathrm{HOO}^{*} \\
\mathrm{HOO}+\mathrm{H}_{2} \mathrm{O}_{2} & \longrightarrow \mathrm{H}_{2} \mathrm{O}+\mathrm{HO}^{+}+\mathrm{O}_{2} \\
\mathrm{HO}^{*}+\text { Polysaccharide- } \mathrm{H} & \longrightarrow \mathrm{H}_{2} \mathrm{O}+\text { Polysaccharide } \\
\text { Polysaccharide+ Monomer } & \longrightarrow \text { Polysaccharide- } g \text {-polymer }
\end{aligned}
$$

Fig. 12: mechanism of the redox reaction involved in the Fenton's reagent

Hydrogen peroxide alone could perform the graft copolymerization, but the use of metallic reducing agents of type $\mathrm{M}^{\mathrm{n}+}\left(\right.$ e.g $\left.\mathrm{Fe}^{2+}\right)$ allows improvements. The redox reactions produce more radicals at significant lower temperatures. Also, chitosan is well known for its chelating properties with metal ions; therefore, the production of hydroxyl radicals could occur closer to the polysaccharidic backbone and enhance the macroradicals formation instead of free vinyl radicals.

Other initiator systems based on a homolytic bond scission have been derivatized and described for the grafting copolymerization of chitin or chitosan. Examples include the following initiators: azobisisobutyronitrile (AIBN), hydrogen peroxide $\left(\mathrm{H}_{2} \mathrm{O}_{2}\right)$, ammonium persulfate (APS) and potassium persulfate (PPS) [114-115]. It is important to underline that persulfate initiators yield anionic persulfate free radicals under thermal or UV conditions that react with the ammonium functionalities of the chitosan chain. This reaction can lead subsequently to glycosidic bond cleavages and oligomeric chitosan [116].

An efficient graft-copolymerization of methyl(methacrylate) and styrene on mercaptochitin by a radical mechanism was reported showing the free radicals issued from a homolytic S-H bond scission of the thiol functionalities of the chain [117]. The synthesis of mercaptochitin and grafting reaction conditions are shown on (Fig. 13) [118].

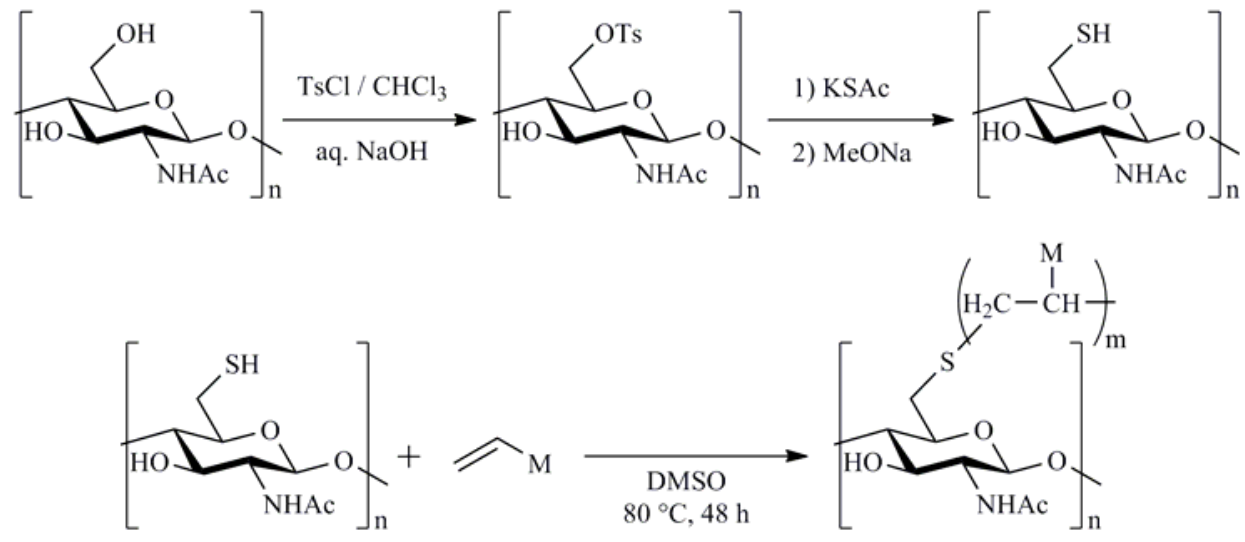

Fig. 13: preparation of mercaptochitin and grafting copolymerization of vinyl monomers 
More recently, a novel redox system based on potassium diperiodatocuprate (III) as oxidant and chitosan as reductant has been published for the copolymerization of methyl acrylate in optimized basic aqueous conditions $\left(\mathrm{pH}_{\text {optimum }}=11.6\right)$ [119]. Low energy barrier for the reductive-oxidative reaction and high grafting yields induce mild reaction conditions and highlight this initiating system as attractive.

\subsubsection{Grafting initiated by radiations}

Another way to generate free radicals able to initiate graft copolymerization of vinylic monomer is to submit an initiator (e.g. AIBN) or modified polysaccharides (e.g. iodochitin) to radiations (UV, microwaves, $\gamma$-radiations). Those compounds have bonds susceptible to undergo homolytic scission and then form free radicals or macroradicals. In case of $\gamma$-radiations, the mechanism appears to be less clearly defined. Electron abstraction occurs on undefined sites along the macromolecule and thus radical cations are produced. Beside this lack of regioselectivity, high-energy radiation initiations exhibit the benefit to require no additional reagents with the monomers.

The use of UV-light to initiate graft copolymerization of styrene onto iodochitin was described [120]. Preparation of iodochitin is a two-step synthesis. Chitin is first converted in tosylchitin as described previously (Fig. 13). Then, into DMSO, tosylchitin reacts with sodium iodide during one day at $85{ }^{\circ} \mathrm{C}$ under nitrogen to form iodochitin [121]. Under UV irradiation, the C6-I bond breaks down and furnishes macroradicals able to initiate the grafting. Later, the copolymerization of hydroxyethyl metacrylate (HEMA) monomers onto chitosan under UV with 4-(benzoyl-benzyl)-trimethylammonium chloride (BTC) as photosensible initiator was outlined $[122]$.

Recently, the microwave irradiation way to initiate graft copolymerization was explored [123]. Without the presence of any initiator or catalyst, poly(acrylonitrile) was successfully grafted onto chitosan. Chitosan-gpoly(acrylonitrile) was obtained within 90 seconds under homogeneous conditions, but with a relatively low grafting yield compared to other techniques previously discussed.

Graft copolymerization of vinyl monomers like styrene, HEMA, $N, N^{\prime}$-dimethylaminoethylmethacrylate (DMAEMA) and butyl acrylate by means of high-energy radiations ( ${ }^{60} \mathrm{CO} \gamma$-radiations) have been extensively reported [124]. The major parameters of grafting were found to be the total radiation dose absorbed, the rate of absorption, the exposition time and the monomer concentration. 


\subsubsection{Grafting via polycondensation}

Graft copolymerization via condensation reaction has been the subject of few studies because of to the polysaccharide sensitivity under high temperatures required to carry out the reaction. Nevertheless, some examples of successful grafting have been reported.

The grafting of D,L-lactic acid (LA) with chitosan was reported in the absence of a catalyst [125]. Polycondensation of L-lactide on phthaloylchitosan primary hydroxyl has been realized by use of DMAP as catalyst [126]. Fig. 14 shows a proposed mechanism of catalytic activation.

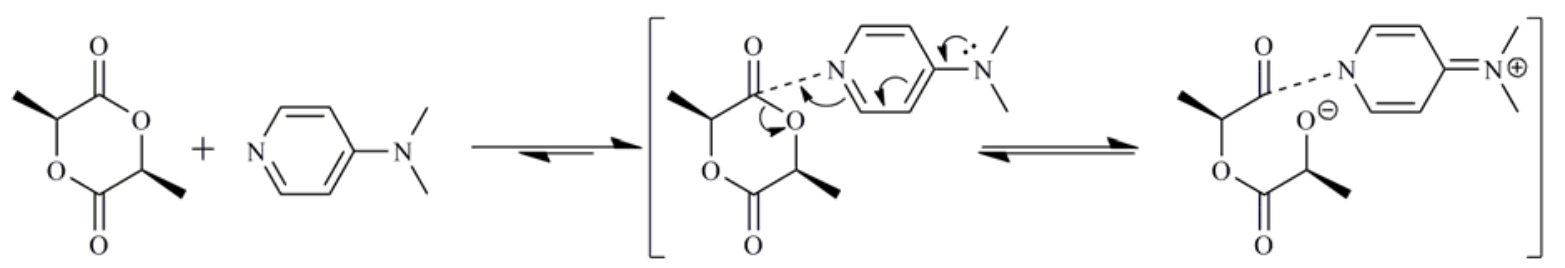

Fig. 14: proposed mechanism for the catalytic activation of L-lactide with DMAP

Condensation of lactobionic acid onto chitosan with EDC and NHS as the coupling agents was performed [127].

\subsubsection{Ring opening polycondensation methods}

Only few kinds of cyclic monomers were subjected to this grafting method. The most widely used was $N$ carboxyanhydrides (NCA) monomer [128]. Cyclic monomers were also described: lactones, 2-alkyl oxazolines and epoxides. The ring-opening polymerization mechanism of NCAs is a nucleophilic attack of the chitosan amino group onto carbonyl A of the NCA. The NCA nitrogen subsequently attacks another anhydride molecule yielding a grafted polypeptide chain and carbon dioxide (Fig. 15) [128a].

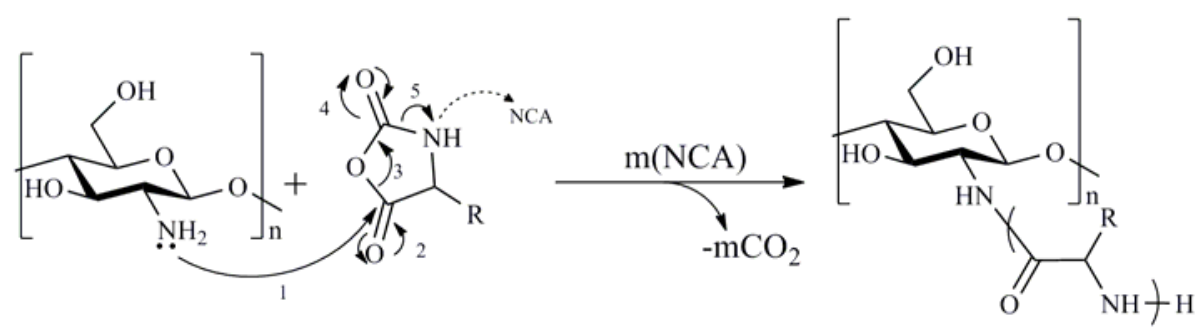

Fig. 15: ring-opening polymerization mechanism of $N$-carboxyanhydrides monomers

Despite low degree of polymerization (DP) for grafted chain length, usually lower than 20 units, this method presents two main advantages: a low homopolymer formation and a control of the side chains length via NCA concentration. 
Another use of the ring opening method was the polymerization of oligo( $\varepsilon$-caprolactone) as side chains onto partially deacetylated chitin. The reaction was led into water (as swelling agent) and catalysed by tin(II) 2ethylhexanoate [129].

Living poly(2-alkyl oxazoline) telechelic polymers was grafted onto a partially deacetylated chitin in DMSO [130]. Cationic ring-opening polymerization of 2-methyl-2-oxazoline were initiated by methyl trifluoromethanesulphonate producing living poly(2-methyl-2-oxazoline) chains. These chains terminated by the amino groups of chitosan yielded the grafted polysaccharide and are analogous to telechelic polymers. Poly(2butyl-2-oxazoline), poly(2-phenyl-2-oxazoline), and poly(isobutylvinyl ether) cation were also successfully grafted onto chitin or chitosan [131]. It is noteworthy that these graft copolymerizations of poly(2alkyloxazoline) can also be named as "grafting onto" methods, see the next part below.

\subsubsection{Grafting onto method}

Unlike the methods previously cited in which the side chains grow from the polysaccharide backbone, grafting onto method is based on the binding of telechelic polymer terminated functions with the macromolecules (Fig. 16). Telechelic polymers have been defined as those containing one or more functional end groups that have the capacity for selective reaction to form bonds with another molecule [132].

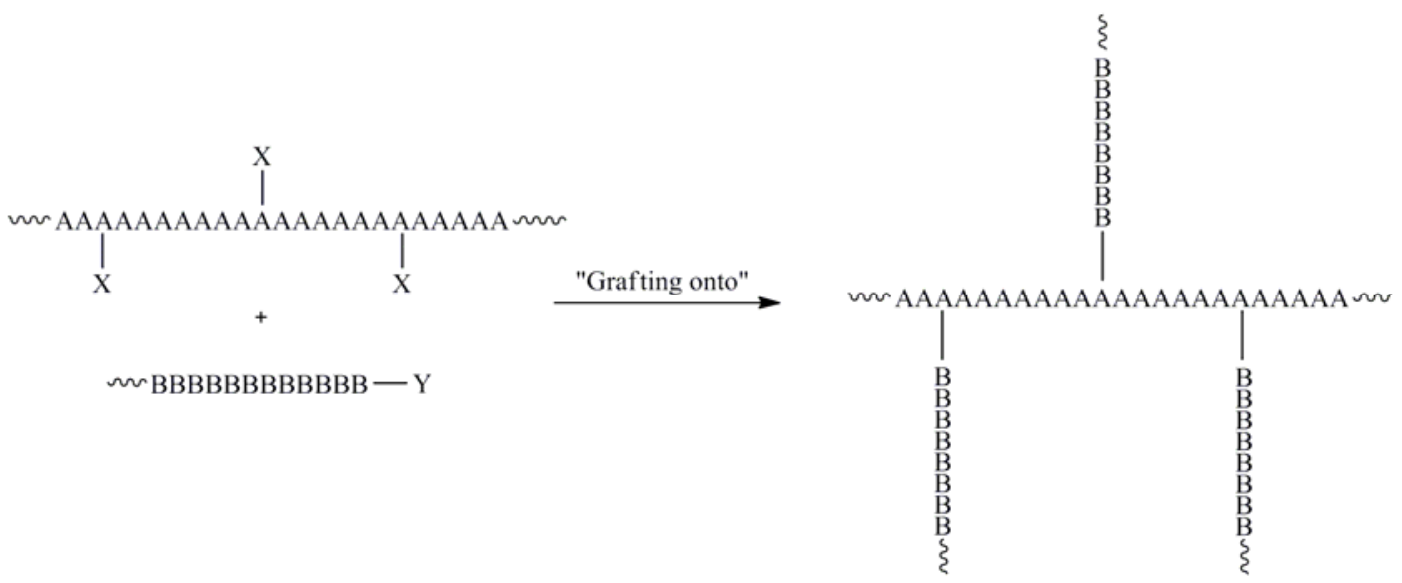

Fig. 16: "grafting onto" method with the final polymer

Different telechelic polymers have been grafted onto chitosan: poly-(ethylene glycol) (PEG), polyurethane, poly(isobutylvinyl ether), polylactide, poly-(ethyleneimine) (PEI), poly-(dimethyl-siloxane) (PDMS), and poly(ethylene oxide)-poly(propylene oxide)-poly-(ethylene oxide) (PEO-PPO-PEO), also known as pluronic polyols or poloxamers $(126,142,143)$. 
Among these, PEGs have been extensively grafted and studied due to their properties in biosciences. Regioselective PEGylations onto the amino group of chitosan or onto its primary hydroxyl groups have been described [134, 139].

Chitosan-6-O-PEG was obtained according to three different synthesis using 6-oxo-2- $\mathrm{N}$-phthaloylchitosan, 6- $\mathrm{O}$ dichlorotriazine-2- $N$-phthaloylchitosan and 3-O-acetyl-2- $N$-phthaloylchitosan intermediates. High degrees of substitution of chitosan $(\approx 90 \%)$ and brush copolymers with high density of PEG attachments were obtained. Another $O$-PEGylation procedure was reported using triazine derivatives as coupling agents with poly-(ethylene glycol) monomethyl ether (MPEG) [133] and with MPEG iodide derivative in order to be released of coupling agents [134].

Numerous syntheses of chitosan- $N$-PEG were published with different kinds of linkages. A technique directly derived from peptide synthesis was used to graft methoxy-poly-(ethylene glycol) acid onto chitosan with an amide linkage via activation thanks to carbodiimide/hydroxybenzotriazole system (Fig. 17) [135]. Grafting of methoxy-poly(ethylene glycol)-p-nitrophenyl carbonate with an urethane linkage was also reported [136].

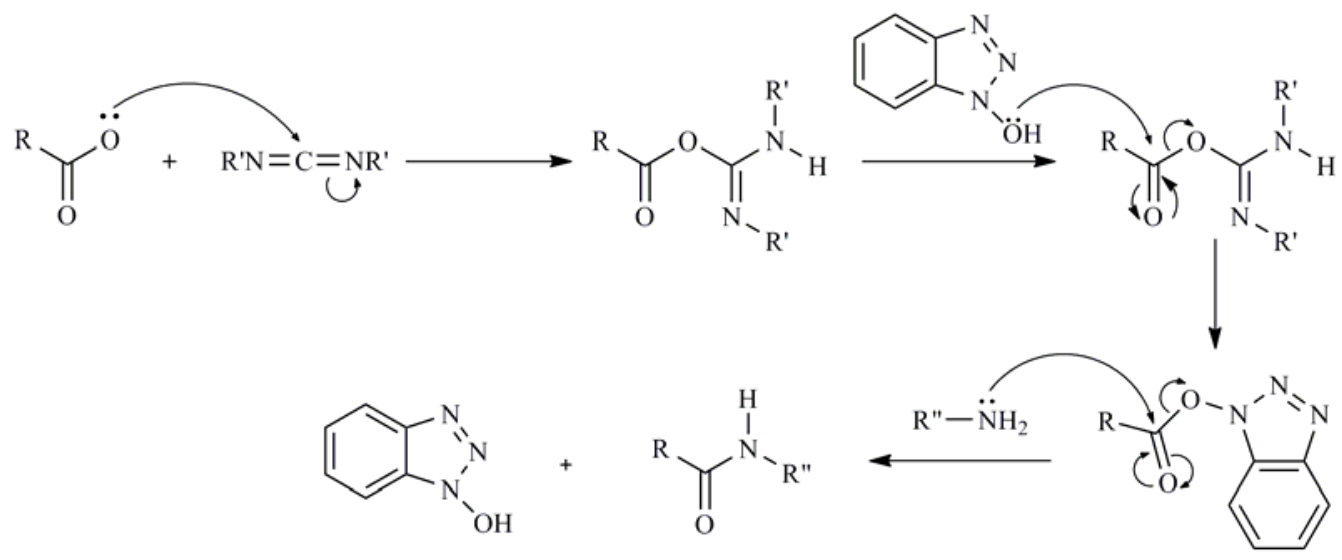

Fig. 17: activation mechanism with the carbodiimide/benzotriazole system

One of the common ways is to follow reductive amination: first, formation of a Schiff base by reaction of chitosan amino group on an aldehyde-terminated PEG; then, reduction by sodium cyanoborohydride $\left(\mathrm{NaBH}_{3} \mathrm{CN}\right)$ leads to a chitosan- $N$-PEG [137]. Direct reductive aminations have been described by using PEG acetaldehyde or PEG propionaldehyde with free amine of chitosan to form an imine bond which can be reduced in stable amine bond [138]. However, some difficulties appear with PEG acetaldehyde use like autopolymerization and air-oxidation. This could be overcome by in situ generating of a PEG acetaldehyde thanks to hydrolysis of a PEG acetaldehyde diethylacetal [139]. 
Other telechelic polymers of interest are polyurethanes obtained from prepolymers bearing polyurethanes groups. The resulting polymer are provided by condensation reactions between poly(ethylene glycol) of two different molar masses and isophorone diisocyanate leading to a NCO terminated polymer [140]. Then, isocyanate functions react with amino groups yielding chitosan- $g$-polyurethane.

Poly(ethylene imine) are linear or branched chains composed of an amine group and two carbon aliphatic as spacer. The grafting proceeds in three steps: first, chitosan chains are crosslinked together with ethylene diglycidyl ether; then, they are functionalized by epichlorohydrin and a final nucleophilic attack of amine functions onto the $\mathrm{C}-\mathrm{Cl}$ bonds leads to the polyaminated chitosan [141].

Pluronic polyols (also known as poloxamers) are constituted of three blocks of polyethers (i.e. PEO-PPO-PEO) and could be grafted onto chitosan [142]. One terminal hydroxyl function of polyethers is activated with $p$ nitrophenyl chloroformate and reacts with chitosan free amino groups giving urethane linkage.

The synthesis and characterization of chitosan-g-poly(dimethyl siloxane) (PDMS) was described. PDMS prepolymers were obtained by ionic ring-opening polymerization of octamethylcyclotrisiloxane using $n$-butyl lithium as initiator [143]. Chlorination with methanesulfonyl chloride provides C6-chlorinated chitosan that can react with the telechelic polymers in order to form the graft copolymers into DMF. Another PDMS prepolymer, terminated with epoxy functions, was grafted onto chitosan using UV irradiation without catalyst at room temperature [144].

\subsubsection{Alginates}

\subsubsection{Grafting initiated by free radicals}

Due to their outstanding properties, alginates and their salts have lots of applications in biomedical fields, purification, food and agriculture. Graft copolymerizations offer the capacity to tune alginates properties by introduction of new chains. As an example, hydrophobicity and steric bulkiness can be introduced in order to protect the polysaccharide from dissolution and erosion.

Taking chitosan as an example, the radical copolymerization of vinyl monomers has been widely reported with various initiating systems. The grafting positions occur usually at the two secondary C2 and C3 hydroxyl groups and at the C6 carboxylic function.

The use of the Fenton's reagent for grafting acrylonitrile (AN) onto alginates was described [145]. A discussion was presented wherein $\mathrm{H}$-atom abstraction on the alginate backbone was reported thanks to the Alg-O-H bond cleavage that results in $\mathrm{Alg}-\mathrm{O} \bullet$ radical. It may however be equally or more likely for $\mathrm{H}$-atom abstraction to occur 
by the ring $\mathrm{C}-\mathrm{H}$ bonds cleavage yielding carbon centered radicals. For alginates as for other polysaccharides, it should be noted that grafting using a free radical initiator like cerium (IV) ammonium nitrate (CAN) or Fenton's reagent is rather unselective, since all $\mathrm{C}-\mathrm{H}$ bonds of the monosaccharide are labile due to the attached oxygen functionality. Therefore, it can be expected that graft initiation points will occur at all monosaccharide carbons [146].

Ceric ion initiated grafting has also been explored with different acrylate monomers like acrylonitrile, methyl acrylate (MA) or methyl methacrylate (MMA) [147]. Highest grafting yields were obtained for AN, followed by MA, followed by MMA. Very recently, the grafting of itaconic acid (IA) onto sodium alginate using CAN / nitric acid $\left(\mathrm{HNO}_{3}\right)$ as redox system to initiate the free radical polymerization was reported [148]. The same grafting copolymerization was published earlier owing to benzoyl peroxide [149]. The use of CAN as initiator was also described to synthesize a biocopolymer composed of alginate and polymethacrylonitrile under aqueous conditions [150].

A superabsorbent composite material was prepared using ammonium persulfate as initiator. The authors grafted simultaneously styrene and a partially neutralized acrylic acid (AA) in water in the presence of attapulgite and with methylenebisacrylamide as crosslinker [151]. More recently, the synthesis of superabsorbent sodium alginate- $g$-poly(sodium acrylate)/polyvinylpyrrolidone and sodium alginate- $g$-poly(acrylic acid)/sodium humate based on the grafting technique initiated by APS was reported [152].

Alginate- $g$-vinyl sulfonic acid graft copolymer was synthesized via the graft copolymerization of vinyl sulfonic acid onto alginate with an efficient system based on potassium peroxydiphosphate and thiourea in an aqueous medium [153]. An initiating system also based on potassium peroxydiphosphate was used towards alginate under inert atmosphere with silver nitrate $\left(\mathrm{AgNO}_{3}\right)$ to polymerize 2-acrylamidoglycolic acid (AGA) that is a class of acrylamides containing $-\mathrm{OH},-\mathrm{COOH}$ and $-\mathrm{CONH}-$ functional groups [154]. Another redox initiator system composed of potassium peroxymonosulphate and glycolic acid allows graft copolymerization of $N$-vinyl2-pyrrolidone under mild condition reaction [155].

Previously described with the chitosan, the novel redox system potassium diperiodatocuprate(III) - sodium alginate was employed to initiate the graft copolymerization of MA in alkali medium [156].

\subsubsection{Grafting initiated by radiations}

Compared to chitosan, alginates are not involved in many researches about radiation-induced polymerization. A microwave-initiated synthesis of alginate- $g$-polyacrylamide in water was reported [157]. 
Recently, a grafting process of poly(acrylamide) onto a blend of starch-chitosan-alginate was carried out in the presence of a low concentration of potassium persulfate initiator and methylenebisacrylamide crosslinker using microwave or UV [158].

It was also reported photo-cured film of sodium alginate with 3-(trimethoxysilyl)propylmethacrylate(silane) in order to improve its physico-mechanical properties [159].

\subsubsection{Grafting onto methods}

One example of the grafting onto method frequently reviewed about alginates and hydrogels is the grafting of poly(N-isopropylacrylamide) (PNIPAAm) to make temperature/pH responsive hydrogels. The $\mathrm{pH}$ dependent response is due to the carboxylic acid groups along the alginate backbone. And the temperature dependent behaviour is well known for poly( $N$-isopropylacrylamide) hydrogels. Alginate- $g$-PNIPAAm hydrogels combine both properties. The grafting occurs into water with the use of EDC and NHS coupling agents between the alginate carboxylic acid functions and the terminal amine of PNIPAAm (Fig. 18) [160].

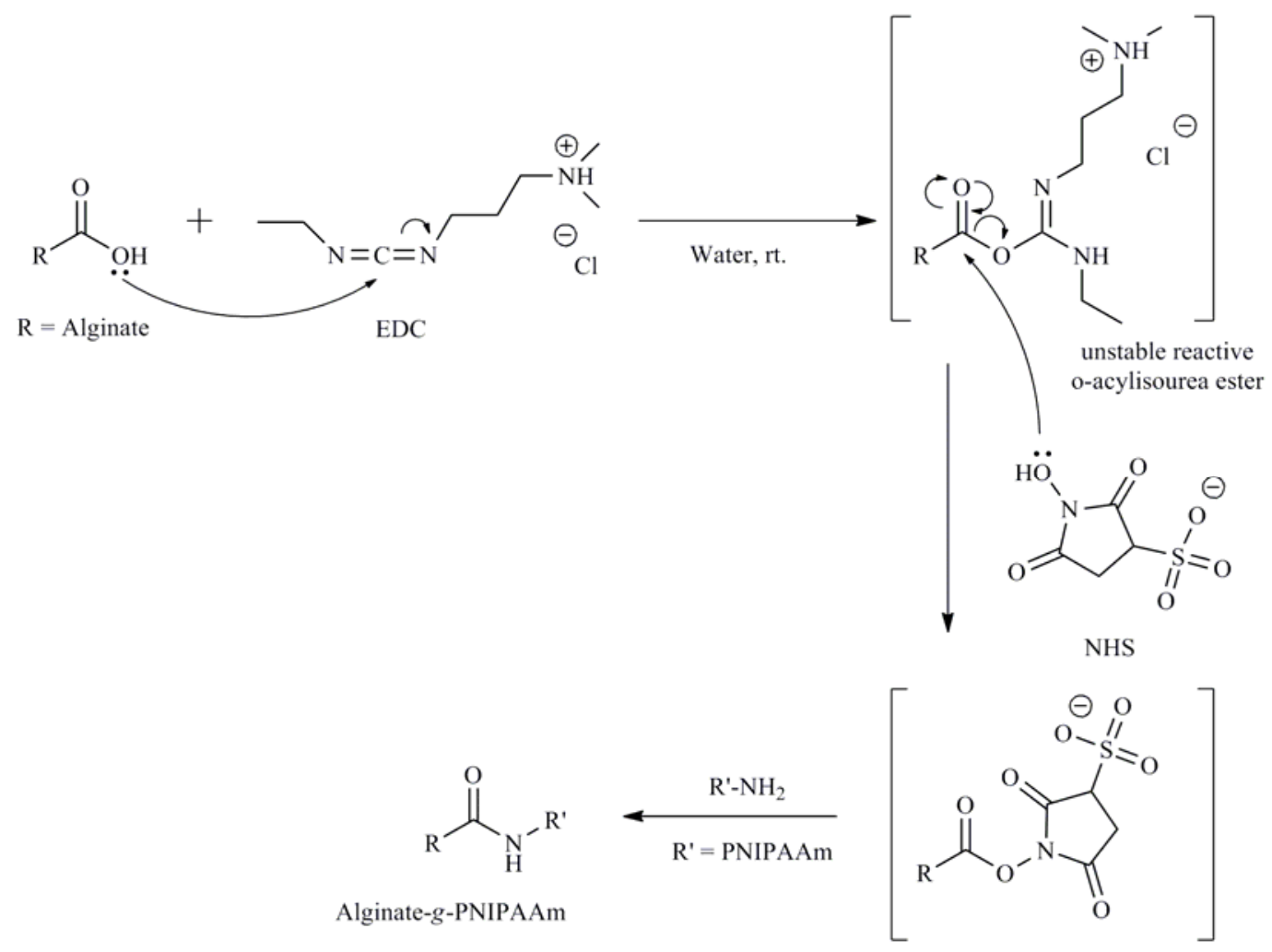

Fig. 18: grafting mechanism with EDC and NHS coupling agents 


\subsubsection{Agar}

\subsubsection{Grafting initiated by free radicals}

Applying CAN redox system, the grafting of methacrylamide and methacrylic acid was performed via free radical graft copolymerization in aqueous and low temperature conditions $\left(30^{\circ} \mathrm{C}\right)[161]$.

\subsubsection{Grafting initiated by radiations}

Grafting of agar and $\kappa$-carrageenan with poly(vinylpyrrolidone) (PVP) chains with average molecular weight at $10 \mathrm{kD}$ in an aqueous medium at $\mathrm{pH} 7$ produced agar- $g$-PVP and $\mathrm{K}$-carrageenan- $g$-PVP blends that can form hydrogels [162]. The reaction was carried out with microwave irradiation in the presence of the water-soluble potassium persulfate (KPS) initiator.

Grafting of polyacrylamide (PAM) chains was performed onto the backbone of agar by microwave assisted method and CAN as the chemical free radical initiator [163].

\subsection{4. $\quad$ K-carrageenans}

\subsubsection{Grafting initiated by free radicals}

A novel biopolymer-based superabsorbent hydrogel was synthesized through chemically crosslinking graft copolymerization of acrylic acid onto $\kappa$-carrageenan, in the presence of a crosslinking agent $(N, N$ '-methylene bisacrylamide (MBA), and APS as free radical initiator [164]. The proposed mechanism for the grafting and chemically crosslinking reactions are outlined in Fig. 19. Thus, the persulfate initiator is decomposed under heating affording sulfate anion-radical. Then, radicals react with hydrogen from the hydroxyl group of the $\mathrm{k}$ carrageenan giving alkoxy radicals on the polymer backbone. Therefore, this "persulfate/saccharide" redox system results in active centers on the substrate to radically initiate polymerization of AA and led to a graft copolymer. Since there is a crosslinking agent in the system, the copolymer has a crosslinked structure. It should be pointed out that the sulfate radical-anion might also initiate AA homopolymerization. 


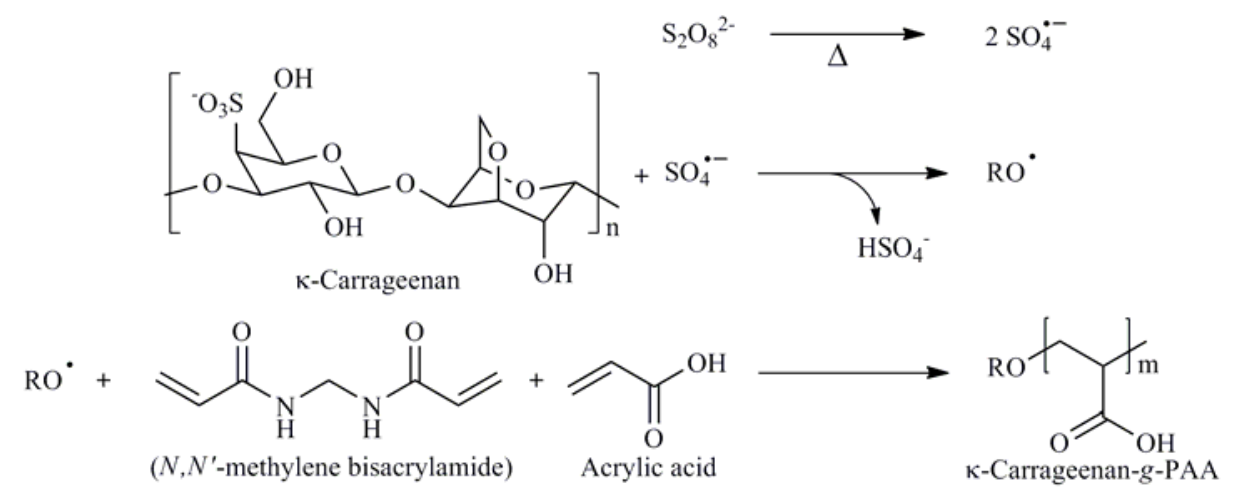

Fig. 19: decomposition of persulfate initiator and grafting of AA in presence of MMB as crosslinker

Few years later, the synthesis of a novel type of highly swelling hydrogels prepared by grafting crosslinked polyacrylic acid-co-poly-2-acrylamido-2-methylpropanesulfonic acid (PAA-co-PAMPS) chains onto $\kappa$ carrageenan was described using the redox system previously mentioned [165].

As for the grafting onto alginate, a reaction was carried out with novel peroxymonosulphate/glycolic acid redox system in order to obtain a carrageenan- $g$ - $N, N$-dimethylacrylamide copolymer under inert atmosphere $[155$, 166].

Peroxymonosulphate and organic acid was extensively during the past few years in order to obtain a variety of grafted $\kappa$-carrageenan. Thereby, the synthesis of these copolymers was reported: $\kappa$-carrageenan- $g$-vinylsulfonic acid, K-carrageenan- $g$-2-acrylamidoglycolic acid and $\kappa$-carrageenan- $g$ - $N$-vinyl formamide using potassium peroxymonosulphate/malonic acid system as redox pair [167]. $\kappa$-carrageenan- $g$ - $N$-vinyl-2-pyrrolidone was also obtained with maleic acid instead of malonic acid [168].

\subsubsection{Grafting initiated by radiations}

A study about the $\gamma$-radiation synthesis of $\kappa$-carrageenan graft copolymers was reported. It consists of blends of $\kappa$-carrageenan with another synthetic polymer to form hydrogels without covalent linkages within the chains.

Thus, the synthesis of $\kappa$-carrageenan-acrylic acid graft copolymers induced by ${ }^{60} \mathrm{Co}-\gamma$ radiations was investigated in order to obtain a precursor of solid catalytic reagent for sucrose hydrolysis [169].

As previously explained, a microwave-assisted synthesis of graft copolymers was applied and yielded a blend of א-carrageenan- $g$-PVP and agar- $g$-PVP. 


\subsection{Cyclic hosts and cyclodextrins}

Some polysaccharides, such as chitosan, are well known for their good chelating capacities for metal ions such as palladium, copper, silver or iodine. In other hands, three classes of compounds are also well known for their great complexing or chelating properties: calixarenes, crown ethers and cyclodextrins (CD) (Fig. 20). Calixarenes are macrocyclic compounds capable of assuming a basket (or "calix") shaped conformation and are constituted of $n$ phenolic units para-substituted. Crown ethers are cyclic chemical compounds that consist of a ring containing several ether groups with at least two carbons between each oxygen atoms. And finally, CDs are cyclic oligosaccharides built from six to eight $D$-glucose units with a specific nomenclature for each $(\alpha=6, \beta=7$, $\gamma=8)$.
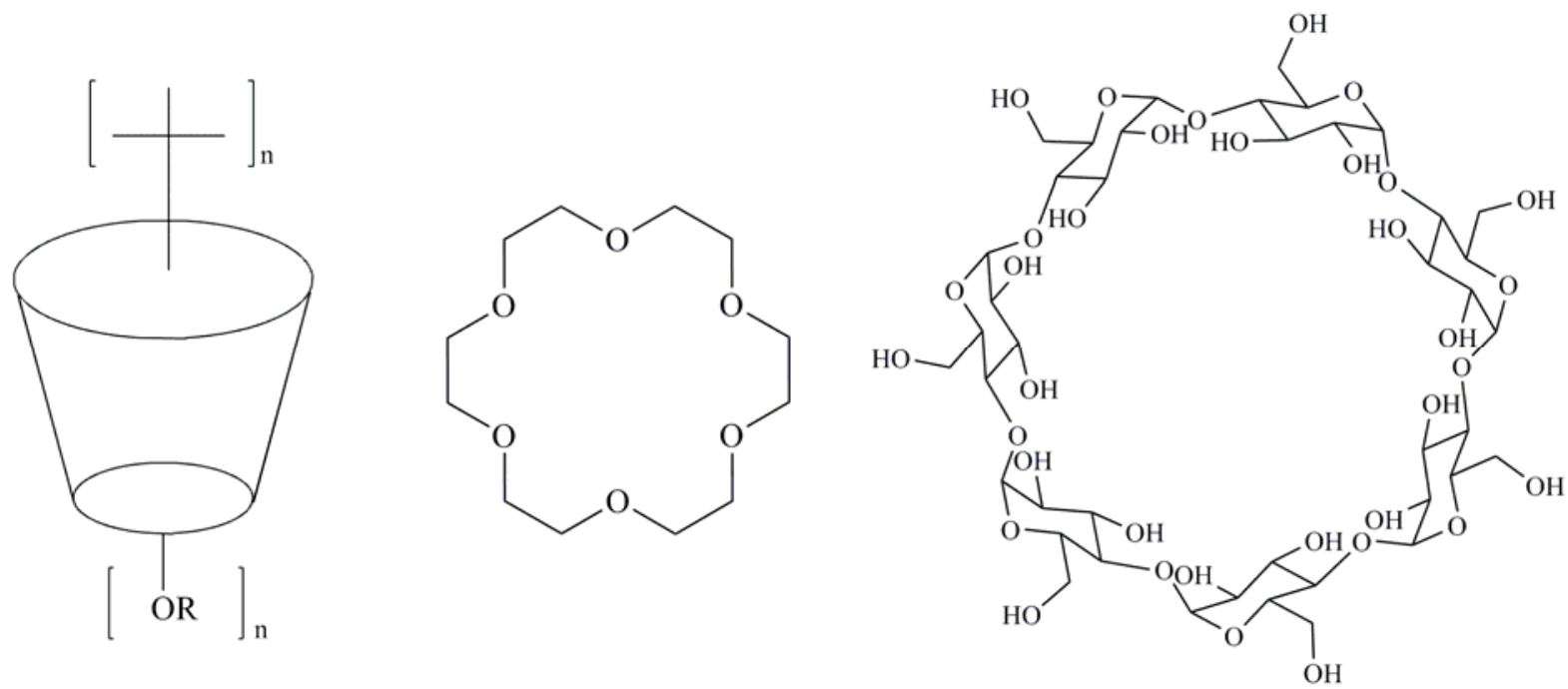

Fig. 20: examples of the three classes of cyclic hosts (A : Calixarenes, B : Crown ethers, $\mathrm{C}$ : Cyclodextrins)

Thus, the combination of these classes of compounds is convenient in order to cumulate their properties. This review will focus on major breakthroughs and the different ways to link cyclic hosts with the polysaccharide backbone. The main fields of applications are, for example, purification of wastewaters, manufacturing solid phases of analytical columns for dosage of specific species or the controlled release of bioactive molecules.

\subsubsection{Chitosan}

\subsubsection{Chitosan / Cyclodextrins}

Different methods have been described utilizing a crosslinker, derivatives of $\mathrm{CD}$ or chitosan. 
The synthesis of a $\beta$-CD-chitosan was presented into aqueous media using EDC without hydroxylbenzotriazole [170]. First, the carboxylic acid functions of carboxymethylated $\beta$-CD react with EDC to form an activated ester intermediate which will subsequently react with the chitosan amino groups in order to create an amide linkage. A modified procedure using $N$-succinylated chitosan was proposed towards $\beta$-CD thanks to EDC into water [171]. The synthesis of $\alpha$ - and $\beta$-CD-chitosan was carried out via reductive amination [172]. Therefore, CD derivatives bearing aldehyde functions react with chitosan amino groups in an acetate buffer at $\mathrm{pH} 4.4$ and room temperature in order to form a Schiff base which is then reduced by sodium cyanoborohydride yielding final amine linkage. Another method is based on 1,6-hexamethylene-di-isocyanate (HMDI), a crosslinker of hydroxyl and amino groups thanks to its two ending isocyanate functions. The reaction is carried under low acidic conditions $(\mathrm{pH}<6)$ in order to make reaction between the chitosan hydroxyl groups and the isocyanate group. The resulting urethane functions are created thanks to the transfer of proton from hydroxyl group to nitrogen atom of isocyanate [173]. Another urethane linkage is generated between isocyanate groups and hydroxyl groups of $\beta$-CD. It is assumed that the crosslinking yields a chitosan-HMDI complex, which reacts afterwards with the hydroxyl groups of $\beta$ CD to form $\beta$-CD-graft-chitosan. HMDI is not able to bind amino groups of chitosan because of its poor affinity for amino groups rather than for hydroxyl groups under acidic conditions [174].

A couple of years later, it was reported a new nucleophilic substitution method to prepare a $\beta$-CD coupled with chitosan [175]. A monochlorotriazinyl derivative of $\beta-\mathrm{CD}$ was employed as a reagent for the grafting onto chitosan owing to a nucleophilic displacement of the chlorine atom.

Another method was noteworthy because of the regioselectivity on position 2 of the D-glucose units of CD. Indeed, secondary hydroxyl groups at the C2 and C3 positions of the glucose units are on the internal side of the torus, as a result these positions are not easily and selectively reachable despite their essential purpose during binding studies. Therefore, CDs bearing good leaving groups such as $p$-toluenesulfonate ester groups were needed. A subsequent reaction between amino groups of the chitosan and these activated CD led to $\beta$-CD-2chitosan derivative [176].

Grafting $\beta$-CD onto the C6 position of the chitosan was also reported using epoxy-activated chitosan [177]. The synthesis has proceeded in four steps (Fig. 21). Firstly, amino groups of chitosan were protected by formation of a Schiff base with benzaldehyde. Under alkali conditions, a subsequent nucleophilic substitution of the chitosan alcoholates groups onto the $\mathrm{Cl}-\mathrm{C}$ bond of the epichlorhydrine led to an epoxy-activated chitosan bearing protective group on amino groups. Then, the benzylidene protective groups of the chitosan derivative were 
cleaved under hydrochloric acid conditions. Lastly, under basic conditions, the cyclodextrin alcoholate groups reacted with an oxirane function giving $\beta-C D-6-c h i t o s a n$.

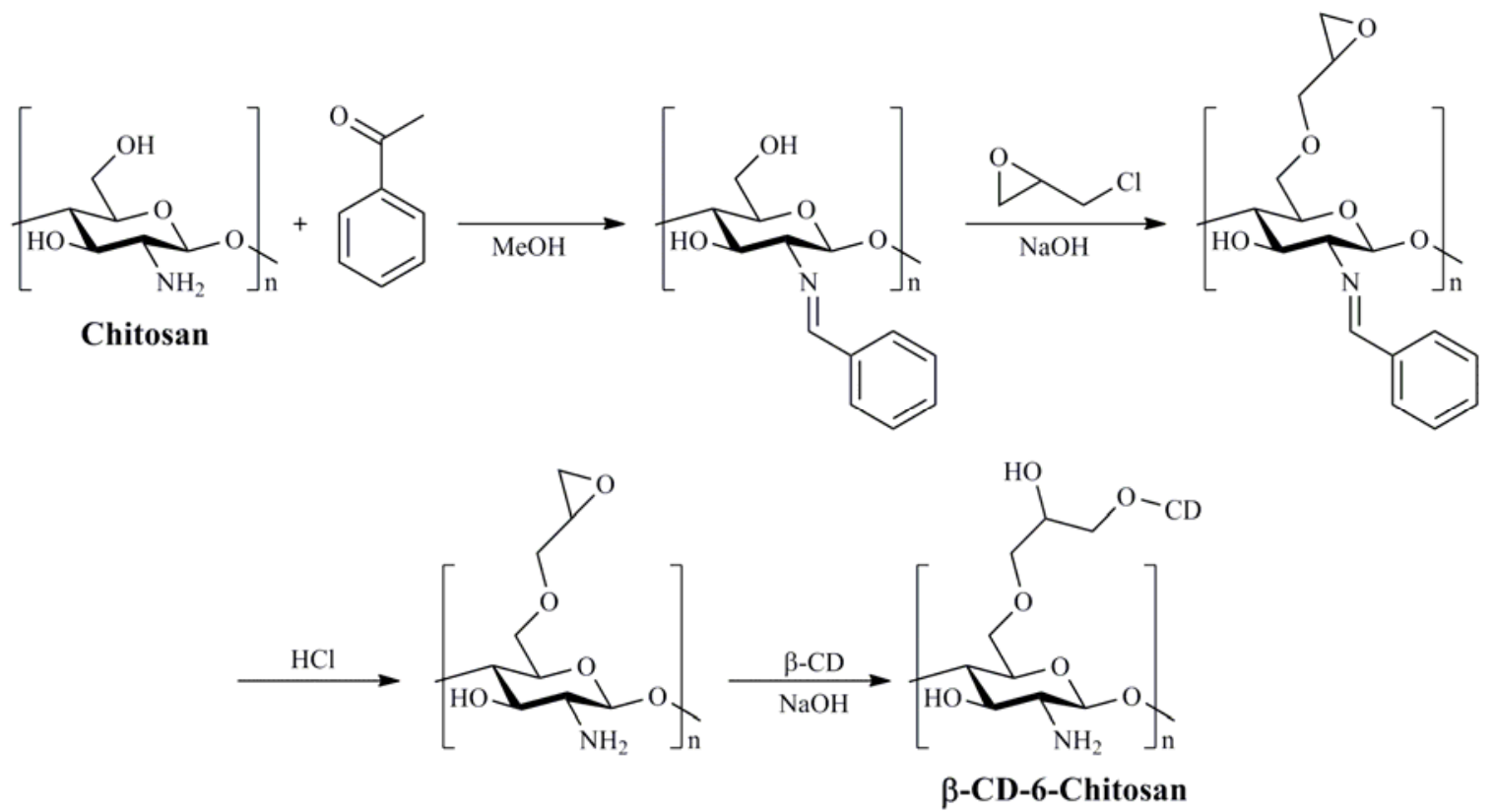

Fig. 21: $\beta$-CD-C6-chitosan obtained in four steps.

At the same time, $\beta$-CD-grafted-chitosan using redox systems was also studied. $\beta$-CD-graft-chitosan was prepared by mixing $\beta-\mathrm{CD}$ itaconate vinyl monomer with chitosan and CAN [178]. The cyclodextrin modified with itaconate was prepared by esterification of the $\beta-\mathrm{CD}$ with itaconic acid in a semi-dry process. The pendant double bonds of $\beta$-CD itaconate were then involved in a free radical graft copolymerization process, described previously in this review.

\subsubsection{Chitosan / Crown ethers or Calixarenes}

Linking methods between crown ethers and calixarenes are analogous to that employed for the cyclodextrins because of their similar reactivity.

The preparation of crown ethers linked onto chitosan was reported [179]. Starting from crown ethers bearing benzaldehyde, chitosan can form a Schiff base or its reduced form (e.g. amino group) [180]. A final basic reaction with the sodium borohydride yielded the amino product (Fig. 22). 


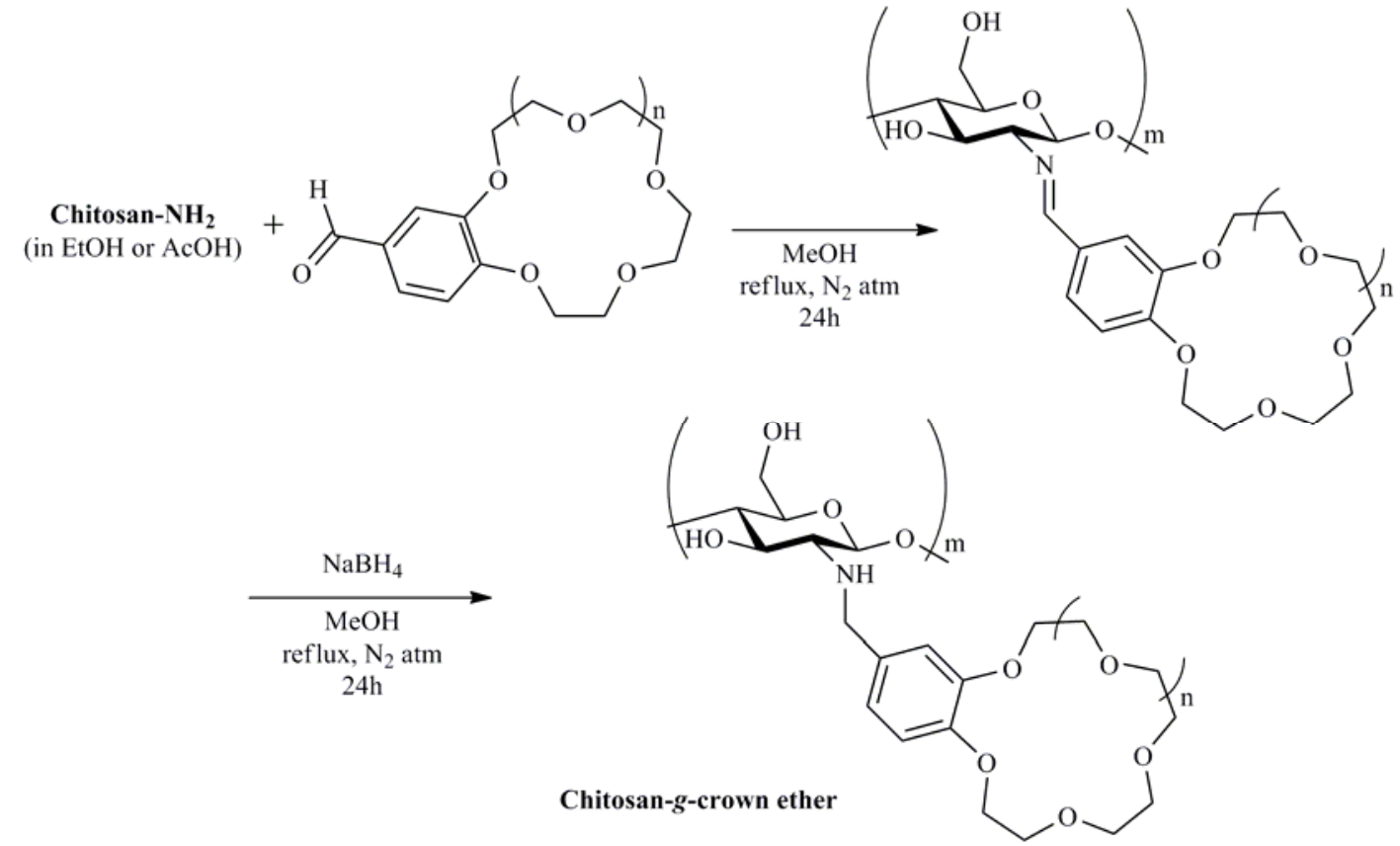

Fig. 22: covalently linked crown ether through amine linkage issued of reductive amination

The next advancement reported in literature was crown ether derivatives that were convenient playing a role as crosslinker and cyclic host [181]. 4,4'-dibromobenzo-18-crown-6 ether was used with chitosan or a $N$-protected chitosan derivative. Unmodified chitosan led to a cross-linked product with heterogeneous structures because of the reaction of both C6 hydroxyl groups and amino groups onto the benzyl bromide moieties. This derivative could form bridges between $\mathrm{C} 6-\mathrm{OH}$ and $\mathrm{C} 6-\mathrm{OH}$, or/and $\mathrm{NH}_{2}$. In order to overcome this drawback, a $N$ benzylidene-protected chitosan derivative bearing available C6 hydroxyl groups was obtained and led to homogeneous crosslinked product at this position.

The synthesis of novel azacrown ether and diazacrown ether chitosan derivatives linked exclusively with the amino groups was described, as well as crosslinking method involving the remaining hydroxyl functions [182]. First, the $N$-allylbenzo-15-crown-5 crown ether was grafted onto chitosan amino group thanks to $\mathrm{FeSO}_{4} / \mathrm{H}_{2} \mathrm{O}_{2}$ redox system, which has initiated the free radical grafting (Fig. 12). The same initiator was employed to graft $N, N$ '-diallyldibenzo 18-crown-6 crown ether but a major crosslinking appears between the chitosan chains because of the allyl groups. A crosslinking was then performed between epichlorhydrine and the chitosan C6 hydroxyl group. A double crosslinking network was created for the diazacrown ether chitosan derivative.

Recently, the microwave irradiation technique was used to obtain a di-Schiff base type crosslinked chitosan dibenzocrown ether via a reaction between the amino and aldehyde groups of chitosan and 4,4-diformyldibenzo- 
18-crown-6 crown ether [183]. This microwave-assisted method is advantageous due to very short time of reaction (ten minutes) compared to conventional synthesis $(24 \mathrm{~h})$ and good yields $(85 \%)$.

A calixarene chitosan derivative was obtained thanks to nucleophilic substitution of chitosan amino groups and hydroxyl groups and the $\mathrm{C}$ - $\mathrm{Cl}$ bond of 1,3-bis-chloroethoxyethoxy-2,4-dihydroxy-p-tert-butylcalix[4]arene or its activated benzoyl derivative (Fig. 23). EDC coupling reagent could also be useful [184].
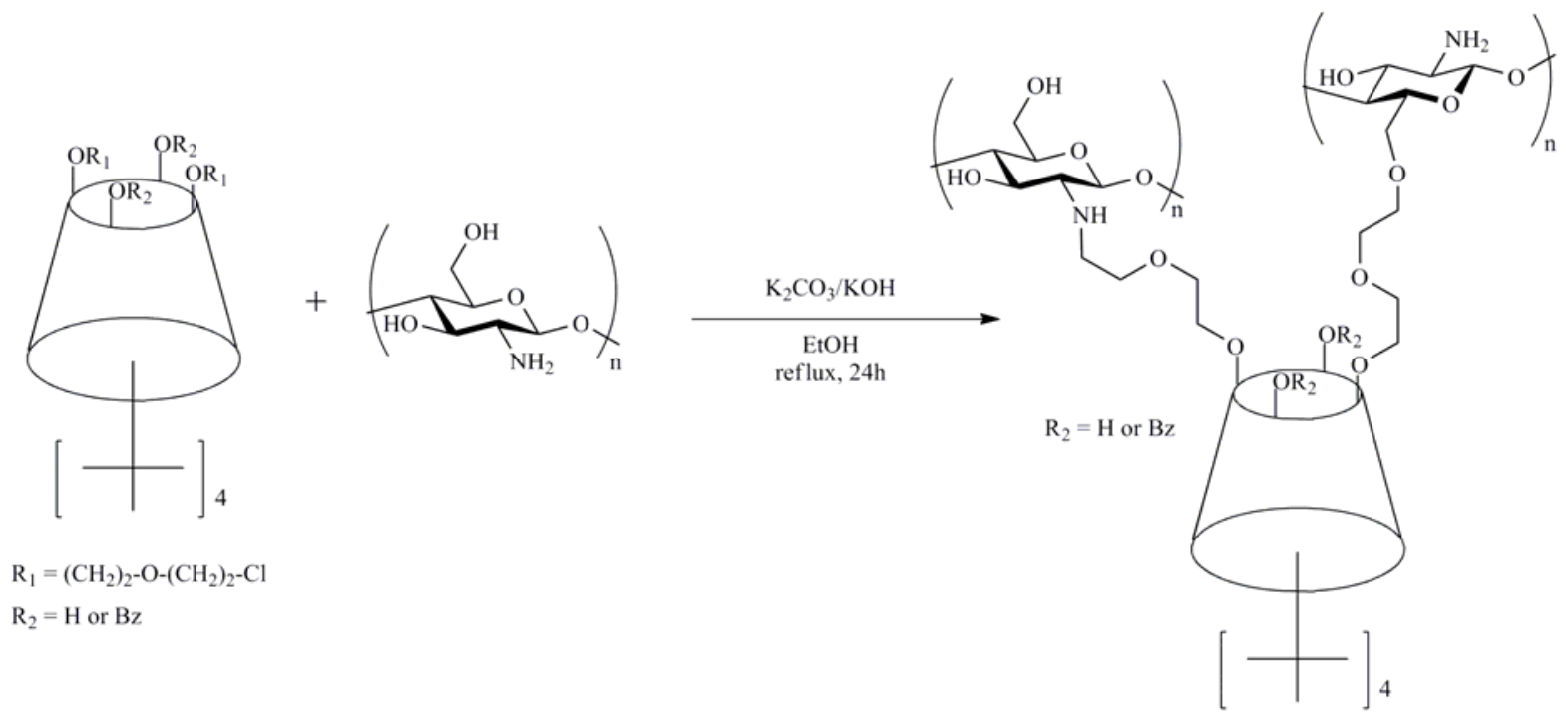

Fig. 23: chitosan-g-calix[4]arene

\subsubsection{Alginates}

A representative method allows the linkage between $\alpha$-cyclodextrins selectively and the alginate hydroxyl groups while preserving its carboxyl groups [185]. Thus, the CNBr method [186] widely used for covalent binding of proteins or enzymes to insoluble polysaccharides (e.g. Sephadex, Sepharose, cellulose) was employed. The alginate derivative bears activated cyano groups and can subsequently react with the amino function carried by the 6-amino- $\alpha$-cyclodextrins yielding carbamimidate group.

\subsubsection{Agar}

The first example found describes CD chemically bound to agar polysaccharide and was very recent [187]. Hydroxypropyl-CD and methyl-CD were cross-linked using ethyleneglycol diglycidylether in alkaline solutions containing agar (up to $1.5 \%$ ) or not. The ethylene glycol diglycidyl ether bears two terminal oxirane functions allowing a crosslinked network between the chemical entities. 
It is noteworthy that blends of $\mathrm{CD}$ and agar have been often mentioned for different applications like electrophoresis or chromatography columns but there were no chemical links between the polysaccharides [188].

\subsection{Dendrimers hybrids}

Dendrimers are a class of hyper-branched molecules with useful applications in biomedical field as inhibitors of the adhesions of pathogenic agents or as DNA transporters.

Chitosan was the first marine polysaccharide used to create a dendrimer hybrid derivative. The surface of chitosan was chemically modified affording a hyperbranched dendritic poly(amidoamine) [189]. The growth of the dendritic poly(amidoamine) was explained as a propagation which started from the surface of the polysaccharide and followed two repeating processes: first, a Michael addition between methyl acrylate and the primary amine group occurred on the surface of chitosan and then an addition of ethylene diamine leaded to amidation of terminal ester groups (Fig. 24).
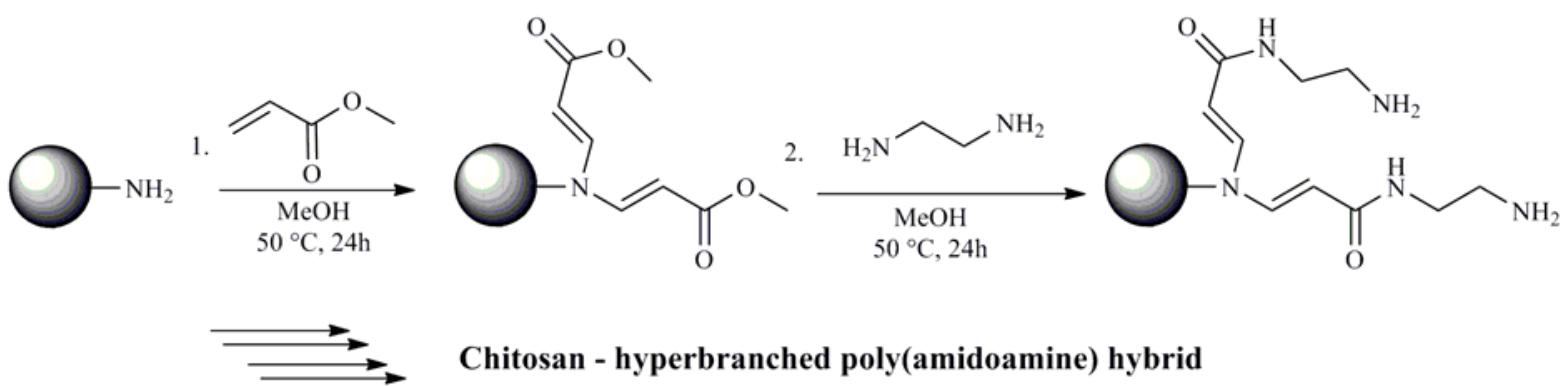

Fig. 24: synthetic pathway to form hyperbranched polyamidoamine grafted onto chitosan

Hyperbranched chitosan-sialic acid dendrimer hybrid with tetraethylene glycol spacer and various other hybrids were also reported [190].

A first technique prepares a dendrimer with a smart use of spacers bearing an aldehyde group that have reacted with chitosan amino group furnishing a Schiff's base. A subsequent reductive amination step with sodium cyanoborohydrure gave the final product [190a]. Applying this reductive amination, no cross-linking occurred during the reaction but some difficulties appeared because of steric hindrance. This method was illustrated by the study of metal binding capacities of these hybrids [191].

Another method used commercially available poly(amidoamine) (PAMAM) and poly(ethylene imine) dendrimers even of high generation (e.g. dendrons nonavalent). However, this synthesis was carried out with 
dendrimers bearing two or more binding groups that might induce crosslinking. This difficulty could be overcome thanks to protective group (e.g. N-methoxycarbonyl-ethyl chitosan derivative) (Fig. 25) [190d].

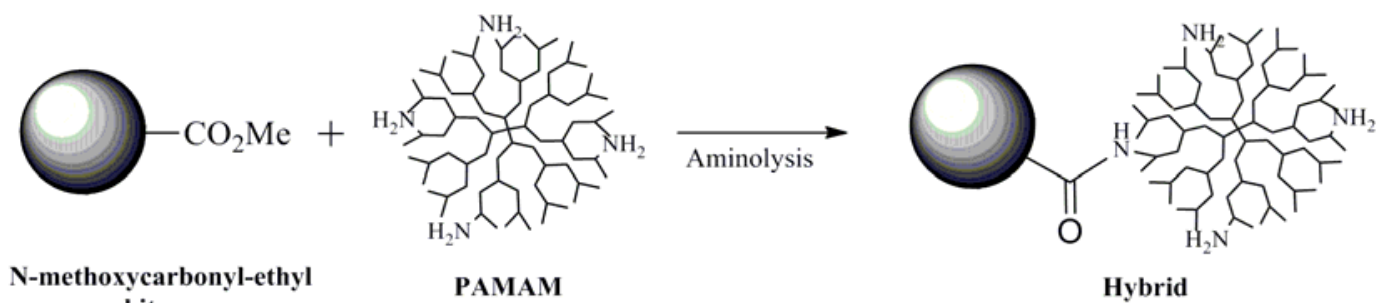
chitosan

Fig. 25: preparation of hybrids Chitosan-dendrimers using commercially available PAMAM

\subsection{Peptide modified}

Major applications of such peptide-bound polysaccharides are based on cell recognition including bacteria and viruses because of specific interactions known between their surfaces (membranes or cell walls) and peptides. Therefore, this kind of substituents enhanced the capacity to mark a defined target.

\subsubsection{Chitin and Chitosan}

Graft copolymerization of amino acids onto partially deacetylated chitin occurred via $\mathrm{N}$-carboxyanhydrides modified amino acids and the polysaccharide backbone amino groups gave a final peptide bound to chitin derivative.

Another graft copolymerization of $\gamma$-methyl-L-glutamate $N$-carboxyanhydride onto partially deacetylated chitin was realized and afforded new polysaccharide/polypeptide hybrid materials with a high control of the polypeptide side-chain length, which had influence on the solubility [192].

Oligopeptide derivative was successfully bound to chitosan through a photoradical mechanism [193] (Fig. 26). Gly-Arg-Gly-Asp (GRGD) oligopeptide reacted with $N$-Succinimidyl-6-[40-azido-20-nitrophenylamino]hexanoate (SANPAH) in a dark room at room temperature for $2 \mathrm{~h}$ furnishing phenyl azidoderivatized peptides. Then, GRGD-SANPAH and chitosan were mixed and dried together; no reaction as yet occurred between both. The chemical linkage was made by a 4 minutes exposition to a UV light (290-370 nm), which created GRGDSANPAH radicals thanks to the decomposition of the azido groups. These radicals reacted onto the chitosan surface yielding an amine linkage between the GRGR-SANPAH derivative and the constitutive glycan of chitosan. 


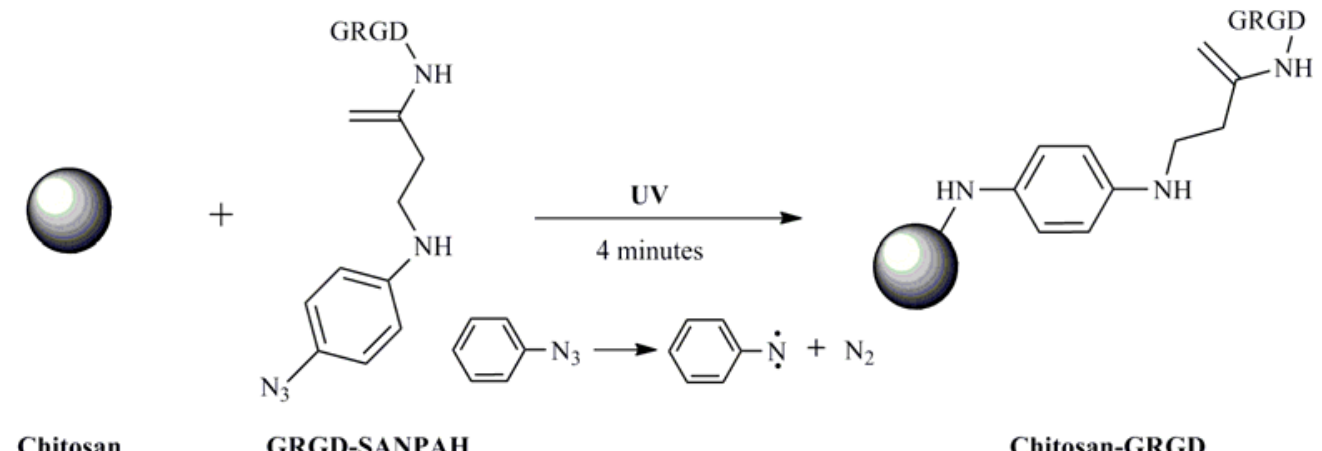

Chitosan

GRGD-SANPAH

Chitosan-GRGD

Fig. 26: UV induced grafting of a peptide derivative onto chitosan

\subsubsection{Alginates}

A hybrid hydrogel of oligopeptide was prepared with alginate for further applications in tissue engineering and particularly bone regeneration [194]. In this study, a novel Bone Morphogenetic Protein-2 (BMP-2) oligopeptide derivative (e.g. NSVNSKIPKACCVPTELSAI) was chemically linked to alginate and implanted into the calf muscle of rats in order to observe ectopic osteoinduction in vivo. In order to produce this hydrogel-like grafted with the BMP-2 derivative, alginate chains were crosslinked with ethylene diamine and EDC producing the targeted scaffold. Then, the remaining carboxyl groups were activated with NHS ester and reacted with BMP-2derived oligopeptide and EDC.

Other alginate-amino acid derivatives were obtained in order to create glycocalyx-like components [195]. The glycocalyx is the external region of a cell membrane that directing specific interaction such as cell-cell recognition and contribute to the steric repulsion that prevents undesirable non-specific adhesions of other molecules and cells. The covalent attachment of amino acid (e.g. L-lysine, L-arginine, L-aspartic acid or Lphenylalanine) to sodium alginate was performed at low temperature $\left(4^{\circ} \mathrm{C}\right)$ using carbodiimide activation of carboxylic acid group by EDC.

A recent study has proposed modification of alginates with a protease-labile double-end grafted crosslinking peptide that could be cleaved by matrix metalloproteinases (MMPs) [196]. The designed MMP-sensitive oligopeptide incorporated the proline-valine-glycine-leucine-isoleucine-glycine (PVGLIG) sequence as the enzymatically cleavable substrate.

Another coupling technique has linked a peptide onto alginate via a carbodiimide linker in a first step followed by the disulfide exchange reaction [197]. The mechanism proposed is an activation of the carboxyl groups of alginate by NHS ester, which could subsequently react with the heterobifunctional reagent 2-(2pyridyldithio)ethyleneamine (PDEA) bearing amino groups and afford thiol-reactive functions along alginate 
chains. The second step is a disulfide exchange between the 2-pyridyldithio sequences and thiol peptides (Fig. 27). The two reaction steps are conducted under mild conditions of temperature and $\mathrm{pH}$. This approach seems very promising for the development of new biofunctionalized alginate-based materials.

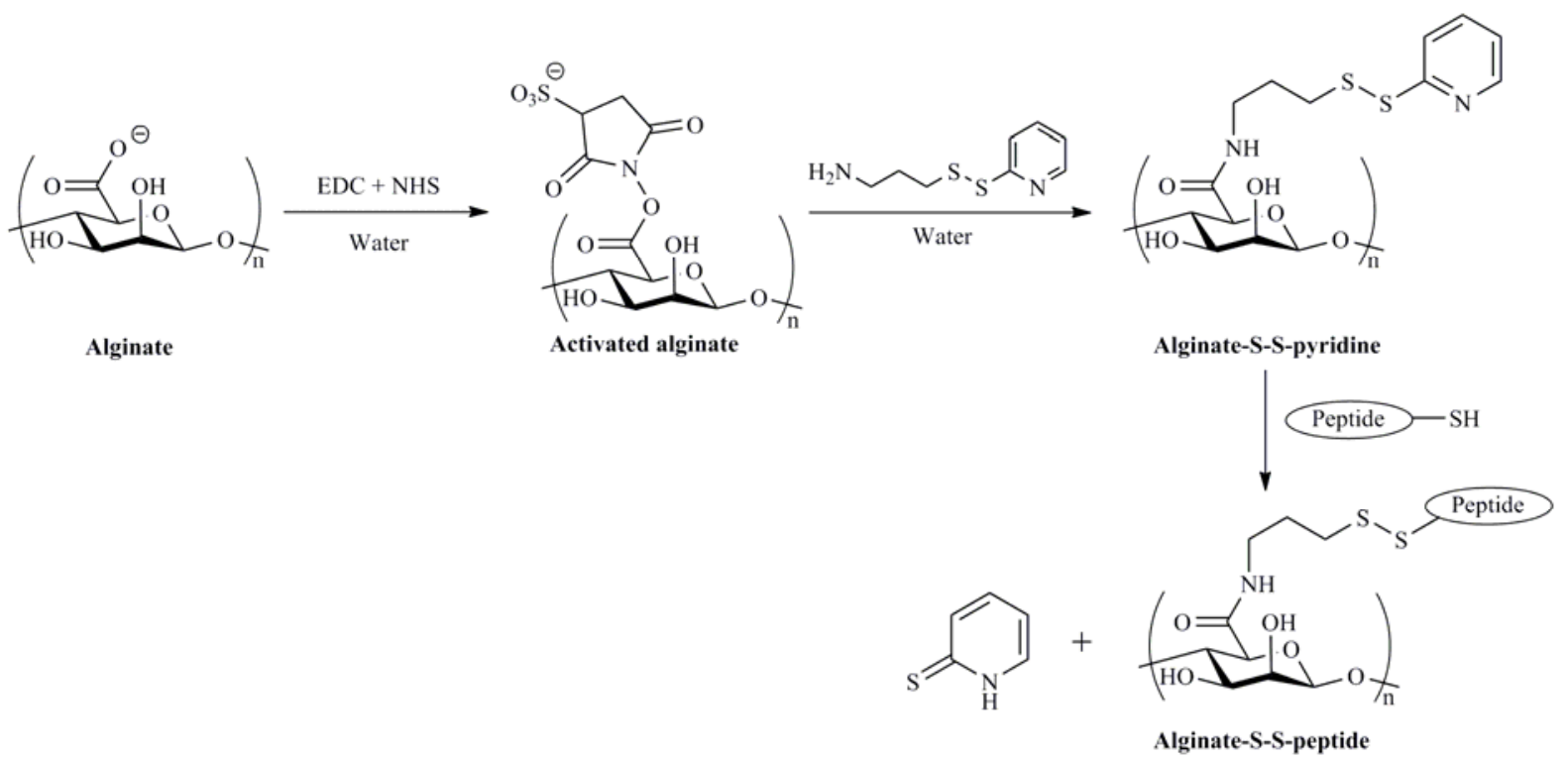

Fig. 27: disulfide exchange with peptide derivative

\subsection{3. $\quad$ Agarose and $\kappa$-carrageenans}

One-pot grafting of agarose and $\kappa$-carrageenan was reported with essential amino-acids cytosine and adenine in order to produce fluorescent polysaccharides [198]. The synthesis has involved a water-based KPS initiated method under microwave irradiation. The proposed microwave activation mechanism involved KPS affording sulfate radical anions, which could then react with polysaccharide primary hydroxyl groups providing macroradicals. The sulfate radical anions reacted also simultaneously in the same way with the amino groups of cytosine or adenine. At the same time, radical, polysaccharide and nucleic base have reacted together leading to amine linkage.

\subsection{Hydrogels and interpenetrating networks}

Hydrogels are defined as three-dimensional (3D) macromolecular networks composed of hydrophilic polymers that swell in water or biological fluids. The main recognized classification separates hydrogels into three classes depending on the nature of their network: entangled networks, covalently crosslinked networks and networks formed by secondary interactions. The last one contains all the intermediary cases between the two previous classes. 
However, this classification was not entirely satisfying concerning polysaccharide hydrogels [199]. A modified classification was suggested for chitosan hydrogels, i.e. chemical and physical hydrogels. Chemical hydrogels were formed by irreversible covalent links like in covalently crosslinked hydrogels and physical hydrogels were created by various reversible links such as ionic interactions (e.g. ionically crosslinked hydrogels and polyelectrolyte complexes) or secondary interactions (e.g. complex hydrogels, grafted chitosan hydrogels and entangled hydrogels).

Extensive reviews dealing with hydrogels and polysaccharide hydrogels have already been published [199, 200], therefore, we will focus on typical example with marine polysaccharides.

\subsubsection{Chemical hydrogels}

Polysaccharide entangled hydrogels and their physico-chemical properties could be improved using a crosslinker.

The next class of hydrogels used functionalized biocompatible polymers with reactive endings in order to form covalently linked networks (AB-graft copolymer). In the role of such biopolymers, PEG-dialdehydes, PEGdiacrylate, telechelic poly(vinyl alcohol) or scleroglucan have been reported [201].

Another class involved the hybrid polymer network (HPN) and semi-interpenetrating polymer network (semiIPN). HPNs were prepared using an additional polymer (e.g. gelatin, collagen) bearing functions, which were able to react with the crosslinker [202] and then have yielded polymeric chains of different nature linked together in a 3D network. Semi-IPNs consist of a polymer bearing no reactive functional sites trapped into a crosslinked network of the second polymer, here the chitosan. The following polymer could be cited: PEG, polyether, PNIPAAm, PVP [152b], gelatin [203] or acrylamide.

Full-IPN was the last possible class of chemically crosslinked networks composed by two polymers and each one was entrapped in its own network through chemical or physical bonds. Different ways to obtain full-IPN existed. If the starting material was a semi-IPN, radiations or a second crosslinker could be used to reticulate the unreacted polymer [204]. It was also possible to obtain an IPN by polymerization of monomers and addition of specific crosslinkers that would allow the formation of the two 3D networks [205]. Fig. 28 presented the different classes of hydrogels. 


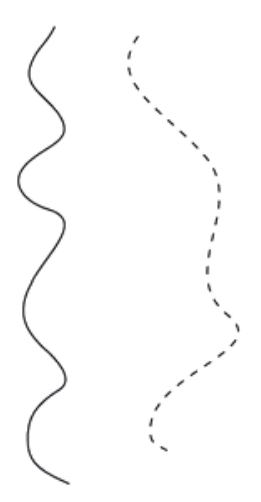

1

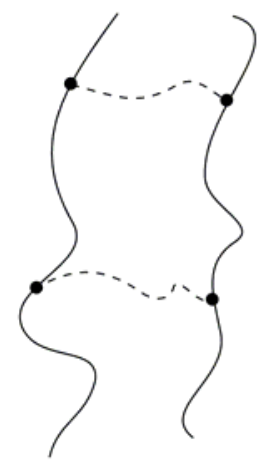

4
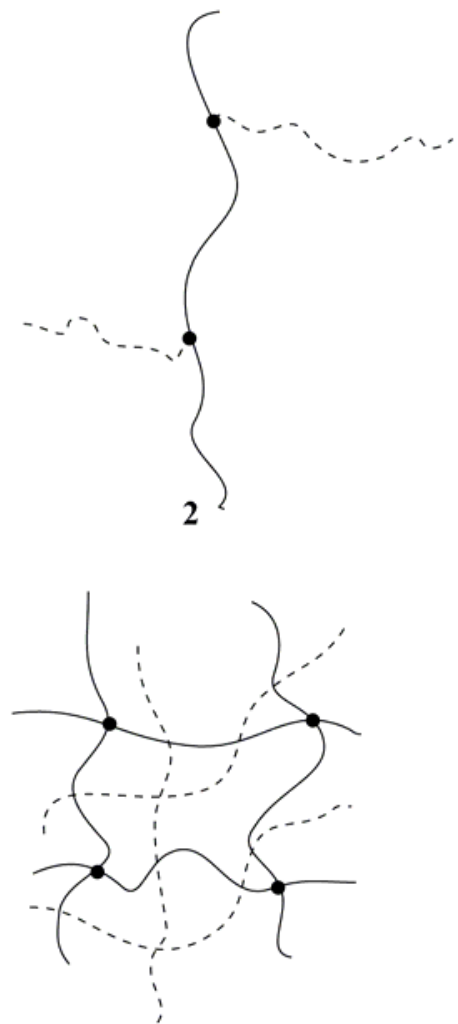

5
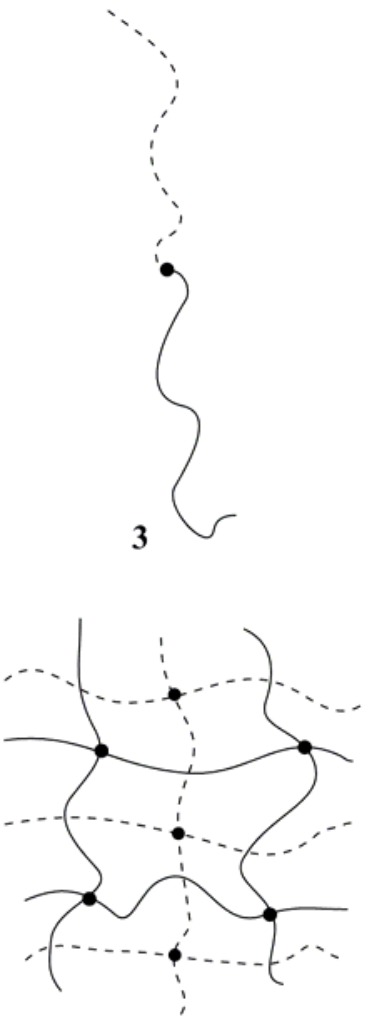

6

Fig. 28: different classes of networks based on two polymers, (1) blend of polymers, (2) graft copolymer, (3) block copolymer, (4) AB-graft copolymer, (5) semi-IPN, (6) full-IPN

\subsubsection{Physical hydrogels}

Physical hydrogels differed of chemical hydrogels by the nature of the linkages between the polymeric chains. As seen above, chemical hydrogels were created by irreversible covalent bonds between chains, whereas physical hydrogels are issued of from reversible linkages with different natures. These hydrogels could present ionic interactions as in the alginate $/ \mathrm{Ca}^{2+}$ ionic crosslinked hydrogel where two carboxylate anions have interacted with one calcium cation [200e]. As observed in the formation of polyelectrolyte complexes, if a polymer bears negative charges and another polymer bears positive charges, ionic interactions occur and create a physical hydrogel. This kind of hydrogel can be classified as follow: for ionic crosslinking, ions or ionic molecules with a well-defined molecular weight and can react with the charged polymers. However, chitosan can react with polymers having large molecular weight distribution in polyelectrolyte complexes [206].

Secondary interactions, or "non-covalent bonding", can also be found as framework. It is the case of some grafted or entangled polysaccharide hydrogels [199]. 


\subsubsection{Examples of marine polysaccharide hydrogels}

Concerning chitosan, dialdehyde crosslinker (e.g. glutaraldehyde, formaldehyde), other crosslinker (e.g. genipin, oxalic acid) have been reported [207]. For the dialdehyde reactant, formation of a Schiff base occurred yielding imine bonds with the amino groups of chitosan but the participation of chitosan hydroxyl groups was not excluded. These reactions could be carried out under aqueous and mild conditions. Thus, the subsequent reductive step was not necessary and this was relevant towards bioapplications. However, dialdehydes were generally considered as toxics. Mechanisms of crosslinking with genipin and oxalic acid were not completely elucidated and oxalic acid has been shown in vitro toxicity in rats [208].

Relating to alginate, epichlorhydrine used as crosslinker in basic aqueous conditions has been described with a mechanism exploiting the two reactive groups of epichlorydrine reagent [146]. However, this reaction produced hydrochloric acid. Glutaraldehyde was also used to form alginate 3D network. As previously discussed, carbodiimide reagents are considered as good candidate to allow covalent crosslinking of alginate chains [146]. Referring to $\kappa$-carrageenan and agar, at least one of the crosslinker cited above has been successfully used and reported [202b].

\section{CONCLUSIONS}

Marine polysaccharides present a growing interest in industrial and pharmaceutical points of view. These abundant natural macromolecules are available from sustainable sea resources because of the real improvements in biotechnology. According to their various origins (animals, seaweeds, invertebrates or microorganisms) marine biopolymers provide original chemical structures such as chitin and its deacetylated derivative chitosan, alginate, carrageenan and agar. Depending on the expected purpose of these materials, some inherent properties can be enhanced, modulated or even induced thanks to chemical modifications.

This review highlighted a wide range of chemical reactions that can be applied to starting marine polysaccharides, which are versatile molecules due to the presence of reactive groups $\left(\mathrm{NH}_{2}, \mathrm{OH}, \mathrm{CO}_{2} \mathrm{H}\right)$ among the polysaccharide backbone. Chemical functionalities can be modified by numerous substitution reactions such as carboxyalkylation, acylation, oxidation, alkylation, sulfation, addition of phosphorous groups, thiolation or "clickable" terminal groups. Marine polysaccharide can also be chemically modified by chain elongation considering polymer chemistry with grafting methods. Cyclic hosts, dendrimer or hydrogel networks can 
promote macromolecular aspect and generate new materials taking advantage of the intrinsic biocompatibility and biodegradability of the polysaccharide scaffold.

Chitosan derivatives have been widely studied due to the two different reactive groups $\left(\mathrm{NH}_{2}, \mathrm{OH}\right)$ on its backbone, which can be distinguished during chemical processes. Nevertheless, other marine polysaccharides have been more recently examined and presented promising properties.

More investigations are needed to demonstrate what kind of interactions, between the modified marine polysaccharides and the biological environment, were responsible for the biological activity. Improved knowledge is needed about the degree of specificity in these interactions, related to a specific reactive group added on the polysaccharide backbone. Nevertheless, it appears that marine biotechnologies have to be taken into account as promising field for sustainable convenient macromolecules. That could address expectation of regenerative medicine, still pending for new biocompatible materials.

\section{ACKNOWLEGMENTS}

This review was financially supported in the frame of BIOREGOS and ANR IONIBIOGEL collaboration projects.

\section{REFERENCES}

[1] Ravi Kumar, M.N.V. A review of chitin and chitosan applications. React. Funct. Polym. 2000, 46, 1-27.

[2] Knutsen, S.H. ; Myladobodski, D.E. ; Larsen, B. ; Usov, A.I. A modified system of nomenclature for red algal galactans. Bot. Mar. 1994, 37, 163-169.

[3] Sova, V. ; Ermakova, S. ; Kuznetsova, T. ; Besednova, N. ; Zaporozhets, T. ; Zvyagintseva, T. Structure, biological activity, and enzymatic transformation of fucoidans from the brown seaweeds. Biotechnol. J. 2008, 3, 904-915.

[4] Berteau, O. ; Mulloy, B. Sulfated fucans, fresh perspectives: structures, functions, and biological properties of sulfated fucans and an overview of enzymes active toward this class of polysaccharide. Glycobiology 2003, 13, 29R-40R.

[5] Lahaye, M.; Robic, A. Structure and Functional Properties of Ulvan, a Polysaccharide from Green Seaweeds. Biomacromolecules 2007, 8, 1765-1774.

[6] a) Kurita, K. Controlled functionalization of the polysaccharide chitin. Prog. Polym. Sci. 2001, 26, 19211971. b) Sashiwa, H. ; Aiba, S.-I. Chemically modified chitin and chitosan as biomaterials. Prog. Polym. Sci. 2004, 29, 887-908. c) Kumar, M.N.V.R. ; Muzzarelli, R.A.A. ; Muzzarelli, C. ; Sashiwa, H. ; Domb, A.J. 
Chitosan Chemistry and Pharmaceutical Perspectives. Chem. Rev. 2004, 104, 6017-6084. d) Kurita, K. Chitin and Chitosan: functional biopolymers from marine crustaceans. Mar. Biotechnol. 2006, 8, 203-226. e) Rinaudo, M. Chitin and chitosan: Properties and applications. Prog. Polym. Sci. 2006, 31, 603-632. f) Alves, N.M. ; Mano, J.F. Chitosan derivatives obtained by chemical modifications for biomedical and environmental applications. Int. J. Biol. Macromol. 2008, 43, 401-414. g) Mourya, V.K. ; Inamdar, N.N. Chitosanmodifications and applications: Opportunities galore. React. Funct. Polym. 2008, 68, 1013-1051. h) Aranaz, I. ; Harris, R. ; Heras, A. Chitosan Amphiphilic Derivatives. Chemistry and Applications. Curr. Org. Chem. 2010, 14, 308-330. i) Jain, A.; Gulbake, A.; Shilpi, S.; Jain, A.; Hurkat, P.; Jain, S.K. A New Horizon in Modifications of Chitosan: Syntheses and Applications. Crit. Rev. Ther. Drug 2013, 30, 91-181.

[7] a) Cathell, M.D.; Szewczyk, J.C. ; Schauer, C.L. Organic Modification of the Polysaccharide Alginate, Mini-Rev. Org. Chem. 2010, 7, 61-67. b) Yang, J.-S. ; Xie, Y.-J. ; He, W. Research progress on chemical modification of alginate: A review. Carbohydr. Polym. 2011, 84, 33-39. c) Pawar, S.N. ; Edgar K.J. Alginate derivatization: A review of chemistry, properties and applications. Biomaterials 2012, 33, 3279-3305. d) Lee, K.Y. ; Mooney, D.J. Alginate: Properties and biomedical applications. Prog. Polym. Sci. 2012, 37, 106- 126. e) Sun, J. ; Tan, H. Alginate-Based Biomaterials for Regenerative Medicine Applications. Materials 2013, 6, 1285 1309.

[8] Campo, V.L. ; Kawano, D.F. ; da Silva Jr., D.B. ; Carvalho, I. Carrageenans: Biological properties, chemical modifications and structural analysis - A review. Carbohydr. Polym. 2009, 77, 167-180.

[9] Pillai, C.K.S. ; Paul, W. and Sharma, C.P. Chitin and chitosan polymers: Chemistry, solubility and fiber formation. Prog. Polym. Sci. 2009, 34, 641-678.

[10] Mourya, V.K. ; Inambar, N.N. and Tiwari, A. Carboxymethyl chitosan and its applications. Adv. Mat. Lett. 2010, 1, 11-33.

[11] Mine, S. ; Izawa, H. ; Kaneko, Y. ; Kadokawa, J. Acetylation of $\alpha$-chitin in ionic liquids. Carbohydr. Res. 2009, 344, 2263-2265.

[12] Sashiwa, H. ; Kawasaki, N. ; Nakayama, A. ; Muraki, E. ; Yamamoto, N. ; Zhu, H. Chemical Modification of Chitosan. 13. Synthesis of Organosoluble, Palladium Adsorbable, and Biodegradable Chitosan Derivatives toward the Chemical Plating on Plastics. Biomacromolecules 2002, 3, 1120-1125.

[13] a) Kurita, K. ; Ikeda, H. ; Shimojoh, M. ; Yang, J. N-Phthaloylated Chitosan as an Essential Precursor for Controlled Chemical Modifications of Chitosan: Synthesis and Evaluation. Polym. J. 2007, 39, 945-952. b) Ifuku, S. ; Miwa, T. ; Morimoto, M. ; Saimoto, H. Preparation of highly chemoselective N-phthaloyl chitosan in aqueous media. Green Chem. 2011, 13, 1499-1502.

[14] Sashiwa, H. ; Kawasaki, N. ; Nakayama, A. ; Muraki, E. ; Yamamoto, N. ; Arvanitoyannis, I. ; Zhu, H. ; Aiba, S. Chemical modification of chitosan 12: synthesis of organo-soluble chitosan derivatives toward palladium absorbent for chemical plating. Chem. Lett. 2002, 598-599.

[15] Carré, M. C. ; Delestre, C. ; Hubert, P. ; Dellacherie, E. Covalent coupling of a short polyether on sodium alginate: Synthesis and characterization of the resulting amphiphilic derivative. Carbohydr. Polym. 1991, 16, 367-379.

[16] Broderick, E. ; Lyons, H. ; Pembroke, T. ; Byrne, H. ; Murray, B. ; Hall, M. The characterisation of a novel covalently modified, amphiphilic alginate derivative, which retains gelling and non-toxic properties. J. Colloid Interf. Sci. 2006, 298, 154-161. 
[17] Yang, L. Q. ; Zhang, B. F. ; Wen, L. Q. (2007). Amphiphilic cholesteryl grafted sodium alginate derivative: Synthesis and self-assembly in aqueous solution. Carbohydr. Polym. 2007, 68, 218-225.

[18] a) Babak, V. G.; Skotnikova, E. A.; Lukina, I. G. ; Pelletier, S.; Hubert, P.; Dellacherie, E. Hydrophobically associating alginate derivatives: Surface tension properties of their mixed aqueous solutions with oppositely charged surfactants. J. Colloid Interf. Sci. 2000, 225, 505-510. b) Boisseson, M. R. D.; Leonard, M. ; Hubert, P. ; Marchal, P. ; Stequert, A. ; Castel, C. ; Favre, E. ; Dellacherie, E. Physical alginate hydrogels based on hydrophobic or dual hydrophobic/ionic interactions: Bead formation, structure, and stability. J. Colloid Interf. Sci. 2004, 273, 131-139. c) Pelletier, S. ; Hubert, P. ; Lapicque, F. ; Payan, E. ; Dellacherie, E. Amphiphilic derivatives of sodium alginate and hyaluronate: Synthesis and physico-chemical properties of aqueous dilute solutions. Carbohydr. Polym. 2000, 43, 343-349.

[19] a) Galant, C. ; Kjøniksen, A. L. ; Nguyen, G. T. M. ; Knudsen, K. D. ; Nyström, B. Altering associations in aqueous solutions of a hydrophobically modified alginate in the presence of $\beta$-cyclodextrin monomers. J. Phys. Chem. B. 2006, 110, 190-195. b) Vallée, F. ; Müller, C. ; Durand, A. ; Schimchowitsch, S. ; Dellacherie, E. ; Kelche, C. ; Cassel, J.-C. ; Leonard, M. Synthesis and rheological properties of hydrogels based on amphiphilic alginate-amide derivatives. Carbohydr. Res. 2009, 344, 223-228.

[20] Kuijpers, A. J.; Engbers, G. H. M.; van Wachem, P. B.; Krijgsveld, J.; Zaat, S. A. J.; Dankert, J.; Feijen, J. Controlled delivery of antibacterial proteins from biodegradable matrices. J. Control. Release 1998, 53, 235-247. [21] Bordenave, N.; Grelier, S.; Coma, V. Advances on Selective C-6 Oxidation of Chitosan by TEMPO. Biomacromolecules 2008, 9, 2377-2382.

[22] Muzzarelli, R.A.A.; Muzzarelli, C.; Cosani, A.; Terbojevich, M. 6-oxychitins, novel hyaluronan-like regiospecifically carboxylated chitins. Carbohydr. Polym. 1999, 39, 361-367.

[23] Kato, Y. ; Kaminaga, J. ; Matsuo, R., Isogai, A. TEMPO-mediated oxidation of chitin, regenerated chitin and N-acetylated chitosan. Carbohydr. Polym. 2004, 58, 421-426.

[24] Yoo, S.H. ; Lee, J.S. ; Park, S. Y. ; Kim, Y. S. ; Chang, P. S. ; Lee, H. G. Effects of selective oxidation of chitosan on physical and biological properties. Int. J. Biol. Macromol. 2005, 35, 27-31.

[25] a) Boontheekul, T. ; Kong, H. ; Mooney, D. Controlling alginate gels degradation utilizing partial oxidation and bimodal molecular weight distribution. Biomaterials, 2005, 26, 2455-2465. b) Kong, H.J. ; Kaigler, D. ; Mooney, D.J. Controlling rigidity and degradation of alginate hydrogels via molecular weight distribution. Biomacromolecules, 2004, 5, 1720-1727.

[26] Gomez, C.G.; Rinaudo, M.; Villar, M. A. Oxidation of sodium alginate and characterization of the oxidized derivatives. Carbohydr. Polym. 2007, 67, 296-304.

[27] de Nooy, A.E.J. ; Capitani, D. ; Masci, G. ; Crescenzi, V. Ionic Polysaccharide Hydrogels via the Passerini and Ugi Multicomponent Condensations: Synthesis, Behavior and Solid-State NMR Characterization. Biomacromolecules, 2000, 1, 259-267.

[28] Bu, H., Kjøniksen, A.L., Knudsen, K.D., Nyström, B. Rheological and structural properties of aqueous alginate during gelation via the Ugi multicomponent condensation reaction. Biomacromolecules, 2004, 5, 14701479.

[29] a) Hirano, S. ; Nagamura, K. ; Zhang, M. ; Kim, S.K. ; Chung, B. G. ; Yoshikawa, M. ; Midorikawa, T. Chitosan staple fibers and their chemical modification with some aldehydes. Carbohydr. Polym. 1999, 38, 293- 
298. b) Moore, G.K.; Roberts, G.A.F. Reactions of chitosan: 3. Preparation and reactivity of Schiff's base derivatives of chitosan. Int. J. Biol. Macromol. 1981, 3, 337-340.

[30] Sashiwa, H. ; Shigemasa, Y. Chemical modification of chitin and chitosan 2: preparation and water soluble property of $N$-acylated or $N$-alkylated partially deacetylated chitins. Carbohydr. Polym. 1999, 39, 127-138.

[31] a) Hall, D. ; Yalpani, M. Formation of branched-chain, soluble polysaccharides from chitosan. J. Chem. Soc., Chem. Commun. 1980, 1153-1154. b) Yalpani, M. ; Hall, D. Some Chemical and Analytical Aspects of Polysaccharide Modifications. 3. Formation of Branched-Chain, Soluble Chitosan Derivatives. Macromolecules 1984, 17, 272-281.

[32] Sashiwa, H. ; Makimura, Y.; Shigemasa, Y. ; Roy, R. Chemical modification of chitosan: preparation of chitosan-sialic acid branched polysaccharide hybrids. Chem. Commun. 2000, 909-910.

[33] Sashiwa, H. ; Thompson, J.M. ; Das, S.K. ; Shigemasa, Y. ; Tripathy, S. ; Roy, R. Chemical modification of chitosan: preparation and lectin binding properties of a-galactosyl-chitosan conjugates. Potential inhibitors in acute rejection following xenotransplantation. Biomacromolecules 2000, 1, 303-305.

[34] Li, D.-H. ; Liu, L.-M. ; Tian, K.-L. ; Liu, J.-C. ; Fan, X.-Q. Synthesis, biodegradability and cytotoxicity of water-soluble isobutylchitosan. Carbohydr. Polym., 2007, 67, 40-45.

[35] Maresh, G. ; Clausen, T. ; Lang, G. In: Chitin and chitosan; Skjak-braek, G. ; Anthonesen, T. ; Sandford, P. Ed.; Elsevier Applied Sciences: London, 1989; pp 389.

[36] a) Ronghua, H. ; Yumin, D. ; Jianhong, Y. Preparation and anticoagulant activity of carboxybutyrylated hydroxyethyl chitosan sulfates. Carbohydr. Polym. 2003, 51, 431-438. b) Xie, Y.; Liu, X. ; Chen, Q. Synthesis and characterization of water-soluble chitosan derivate and its antibacterial activity. Carbohydr. Polym. 2007, $69,142-147$.

[37] Tokura, S. ; Nishi, N. ; Tsutsumi, A. ; Somorin, O. Studies on chitin VIII. Some properties of water soluble chitin derivatives. Polym. J. 1983, 15, 485-489.

[38] a) Domard, A. ; Rinaudo, M. ; Terrassin, C. New method for the quaternization of chitosan. Int. J. Biol. Macromol. 1986, 8, 105-107. b) Sieval, A.B. ; Thanou, M. ; Kotze, A.F. ; Verhoef, J.C. ; Brussee, J. ; Junginger, H.E. Preparation and NMR characterization of highly substituted $N$-trimethylchitosan chloride. Carbohydr. Polym. 1998, 36, 157-165.

[39] Snyman, D. ; Hamman, J.H. ; Kotze, J.S. ; Rollings, J.E.; Kotze, A.F. The relationship between the absolute molecular weight and the degree of quaternization of $N$-trimethyl chitosan chloride. Carbohydr. Polym. 2002, 50, 145-150.

[40] a) Polnok, A.; Borchard, G.; Verhoef, J.C.; Sarisuta, N. ; Junginger, H.E. Influence of methylation process on the degree of quaternization of $N$-trimethyl chitosan chloride. Eur. J. Pharm. Biopharm. 2004, 57, 7783. b) Curti, E. ; De Britto, D. ; Campana-Filho, S.P. Methylation of chitosan with iodomethane: effect of reaction conditions on chemoselectivity and degree of substitution. Macromol. Biosci. 2003, 3, 571-576.

[41] Di Colo, G. ; Burgalassi, S. ; Zambito, Y. ; Monti, D. ; Chetoni, P. Effects of different $N$-trimethyl chitosans on in vitro/in vivo ofloxacin transcorneal permeation. J. Pharm. Sci. 2004, 93, 2851-2862.

[42] Verheul, R.J. ; Amidi, M. ; Van Der Wal, S. ; Van Riet, E. ; Jiskoot, W. ; Hennink, W. E. Synthesis, characterization and in vitro biological properties of $O$-methyl free $N, N, N$-trimethylated chitosan. Biomaterials 2008, 29, 3642-3649. 
[43] De Britto, D. ; Assis, O.B.G. A novel method for obtaining a quaternary salt of chitosan. Carbohydr. Polym. 2007, 69, 305-310.

[44] a) Jia, Z.; Shen, D. ; Xu, W. Synthesis and antibacterial activities of quaternary ammonium salt of chitosan. Carbohydr. Res. 2001, 333, 1-6. b) Avadi, M.R.; Sadeghi, A.M.M.; Tahzibi, A.; Bayati, K.; Pouladzadeh, M. ; Zohuriaan-Mehr, M.J. ; Rafiee-Tehrani, M. Diethylmethyl chitosan as an antimicrobial agent: synthesis, characterization and antibacterial effects. Eur. Polym. J. 2004, 40, 1355-1361. c) Guo, Z. ; Xing, R. ; Liu, S. ; Zhong, Z. ; Ji, X. ; Wang, L. ; Li, P. Antifungal properties of Schiff bases of chitosan, N-substituted chitosan and quaternized chitosan. Carbohydr. Res. 2007, 342, 1329-1332.

[45] Cho, J. ; Grant, J. ; Piquette-Miller, M. ; Allen, C. Synthesis and physicochemical and dynamic mechanical properties of a water-soluble chitosan derivative as a biomaterial. Biomacromolecules 2006, 7, 2845-2855.

[46] Xu, T.; Xin, M. ; Li, M. ; Huang, H. ; Zhou, S. ; Liu, J. Synthesis, characterization, and antibacterial activity of N,O-quaternary ammonium chitosan. Carbohydr. Res. 2011, 346, 2445-2450.

[47] Bhaskar, U.; Sterner, E.; Hickey, A.M.; Onishi, A.; Zhang, F.; Dordick J.S.; Linhardt, R.J. Engineering of routes to heparin and related polysaccharides. Appl. Microbiol. Biotechnol. 2012, 93, 1-16.

[48] a) Guerrini, M.; Beccati, D.; Shriver, Z.; Naggi, A.; Viswanathan, K.; Bisio, A.; Capila, I.; Lansing, J.C.; Guglieri, S.; Fraser, B.; Al-Hakim, A.; Gunay, N.S.; Zhang, Z.; Robinson, L.; Buhse, L.; Nasr, M.; Woodcock, J.; Langer, R.; Venkataraman, G.; Linhardt, R.J.; Casu, B.; Torri, G.; Sasisekharan, R. Oversulfated chondroitin sulfate is a contaminant in heparin associated with adverse clinical events. Nat. Biotechnol. 2008, 26, 669-675. b) Pan, J.; Qian, Y.; Zhou, X.; Pazandak, A.; Frazier, S.B.; Weiser, P.; Lu, H.; Zhang, L. Oversulfated chondroitin sulfate is not the sole contaminant in heparin. Nat. Biotechnol. 2010, 28, 203-207. c) Guerrini, M.; Shriver, Z.; Naggi, A.; Casu, B.; Linhardt, R.J.; Torri, G.; Sasisekharan, R. Reply to Oversulfated chondroitin sulfate is not the sole contaminant in heparin. Nat. Biotechnol. 2010, 28, 207-211.

[49] US Food and Drug Administration (FDA) website

(http://www.fda.gov/Drugs/DrugSafety/PostmarketDrugSafetyInformationforPatientsandProviders/ucm112669.h tm, Accessed October 28, 2013)

[50] a) Senni, K.; Pereira, J.; Gueniche, F.; Delbarre-Ladrat, Sinquin, C.; Ratiskol, J.; Godeau, G.; Fischer, A.M.; Helley, D.; Colliec-Jouault, S. Marine Polysaccharides: A Source of Bioactive Molecules for Cell Therapy and Tissue Engineering. Mar. Drugs, 2011, 9, 1664-1681. b) Laurienzo, P. Marine Polysaccharides in Pharmaceutical Applications: An Overview. Mar. Drugs, 2010, 8, 2435-2465.

[51] Al-Horaniand, R.A.; Desai, U.R. Chemical sulfating of small molecules - advances and challenges. Tetrahedron 2010, 66, 2907-2918.

[52] a) Melo, M.R.S.; Feitosa, J.P.A.; Freitas, A.L.P.; de Paula, R.C.M. Isolation and characterization of soluble sulfated polysaccharide from the red seaweed Gracilaria cornea. Carbohydr. Polym. 2002, 49, 491-498. b) Qi, H.M.; Zhang, Q.B.; Zhao, T.T.; Chen, R.; Zhang, H.; Niu, X.Z.; Xing, R.E. Antioxidant activity of different sulfate content derivatives of polysaccharide extracted from Ulva pertusa (Chlorophyta) in vitro. Int. J. Biol. Macromol. 2005, 37, 195-199. c) Yu, P.Z.; Li, N.; Liu, X.G.; Zhou, G.F.; Zhang, Q.B.; Li, P.C. Antihyperlipidemic effects of different molecular weight sulfated polysaccharides from Ulva pertusa (Chlorophyta). Pharmacol. Res. 2003, 48, 543-549. d) Zhao, T.T.; Zhang, Q.B.; Qi, H.M.; Zhang, H.; Niu, X.Z.; Xu, Z.H.; Li, Z.E. Degradation of porphyran from Porphyra haitanensis and the antioxidant activities of the degraded porphyrans with different molecular weight. Int. J. Biol. Macromol. 2006, 38, 45-50. e) Zhao, T.T.; 
Zhang, Q.B.; Qi, H.M.; Liu, X.G.; Li, Z.E. Extension of life span and improvement of vitality of Drosophila melanogaster by long-term supplementation with different molecular weight polysaccharides from Porphyra haitanensis. Pharmacol. Res. 2008, 57, 67-72.

[53] Jayakumar, R. ; Nwe, N. ; Tokura, S. ; Tamura, H. Sulfated chitin and chitosan as novel biomaterials. Int. J. Biol. Macromol. 2007, 40, 175-181.

[54] Gilbert, E.E. The reactions of sulfur trioxide, and its adducts, with organic compounds. Chem. Rev. 1962 , $62,549-589$.

[55] Zou, Y. ; Khor, E. Preparation of sulfated-chitins under homogeneous conditions. Carbohydr. Polym. 2009, $77,516-525$.

[56] Huang, R. ; Du, Y. ; Yang, J. ; Fan, L. Influence of functional groups on the in vitro anticoagulant activity of chitosan sulfate. Carbohydr. Res. 2003, 338, 483-489.

[57] Nagasawa, K. ; Tohira, Y. ; Inoue, Y. ; Tanoura, N. Carbohydr. Res. 1971, 18, 95-102.

[58] Vikhoreva, G. ; Bannikova, G. ; Stolbushkina, P. ; Panov, A. ; Drozd, N. ; Makarov, V. ; Varlamov, V. ; Gal'braikh, L. Carbohydr. Polym. 2005, 62, 327-332.

[59] Zhang, C. ; Ping, Q. ; Zhang, H. ; Shen, J. Preparation of $N$-alkyl- $O$-sulfate chitosan derivatives and micellar solubilization of taxol. Carbohydr. Polym. 2003, 54, 137-141.

[60] Hirano, S. ; Tanaka, Y.; Hasegawa, M. ; Tobetto, K. ; Nishioka, A. Effect of sulfated derivatives of chitosan on some blood coagulant factors. Carbohydr. Res. 1985, 137, 205-215.

[61] Gamzazade, A. ; Sklyar, A. ; Nasibov, S. ; Sushkov, I. ; Shashkov, A. ; Knirel, Y. Structural features of sulfated chitosans. Carbohydr. Polym. 1997, 34, 113-116.

[62] Je, J.-Y. ; Park, P.-J. ; Kim, S.-K. Prolyl endopeptidase inhibitory activity of chitosan sulfates with different degree of deacetylation. Carbohydr. Polym. 2005, 60, 553-556.

[63] Yang, J. ; Luo, K. ; Li, D. ; Yu, S. ; Cai, J. ; Chen, L. ; Du, Y. Preparation, characterization and in vitro anticoagulant activity of highly sulfated chitosan. Int. J. Biol. Macromol. 2013, 52, 25-31.

[64] Suwan, J. ; Zhang, Z. ; Li, B. ; Vongchan, P. ; Meepowpan, P. ; Zhang, F. ; Mousa, S.A. ; Mousa, S. ; Premanode, B. ; Kongtawelert, P. ; Linhardt, R.J. Sulfonation of papain-treated chitosan and mechanism for anticoagulant activity. Carbohydr. Res. 2009, 344, 1190-1196.

[65] a) Nishimura, S.-I. ; Kai, H. ; Shinada, K. ; Yoshida, T. ; Tokura, S. ; Kurita, K. ; Nakashima, H. ; Yamamoto, N. ; Uryu, T. Regioselective syntheses of sulfated polysaccharides: specific anti-HIV-1 activity of novel chitin sulfates. Carbohydr. Res. 1998, 306, 427-433. b) Nishimura, S. ; Miura, Y. ; Ren, L. ; Sato, M. ; Yamagishi, A. ; Nishi, N. ; Tokura, S. ; Kurita, K. ; Ishii, S. An Efficient Method for the Syntheses of Novel Amphiphilic Polysaccharides by Regio- and Thermoselective Modifications of Chitosan. Chem. Lett. 1993, $1623-1626$.

[66] Muzzarelli, R.A.A. Modified chitosans carrying sulfonic acid groups. Carbohydr. Polym. 1992, 19, 231236.

[67] Alban, S. ; Schauerte, A. ; Franz, G. Anticoagulant sulfated polysaccharides: part I. Synthesis and structure activity relationships of new pullulan sulfates. Carbohydr. Polym. 2002, 47, 267-276.

[68] Ronghua, H. ; Yumin, D. ; Jianhong, Y. Preparation and in vitro anticoagulant activities of alginate sulfate and its quaterized derivatives. Carbohydr. Polym. 2003, 52, 19-24. 
[69] Freeman, I. ; Kedem, A. ; Cohen, S. The effect of sulfation of alginate hydrogels on the specific binding and controlled release of heparin-binding proteins. Biomaterials 2008, 29, 3260-3268.

[70] Fan, L. ; Jiang, L. ; Xu, Y. ; Zhou, Y. ; Shen, Y. ; Xie, W. ; Long, Z. ; Zhou, J. Synthesis and anticoagulant activity of sodium alginate sulfates. Carbohydr. Polym. 2011, 83, 1797-1803.

[71] Jayakumar, R.; Selvamurugan, N. ; Nair, S.V.; Tokura, S.; Tamura, H. Preparative methods of phosphorylated chitin and chitosan - An overview. Int. J. Biol. Macromol. 2008, 43, 221-225.

[72] Nishi, N. ; Ebina, A.; Nishimura, S. ; Tsutsumi, A. ; Hasegawa, O. ; Tokura, S. Highly phosphorylated derivatives of chitin, partially deacetylated chitin and chitosan as new functional polymers: preparation and characterization. Int. J. Biol. Macromol. 1986, 8, 311-317.

[73] Sakaguchi, T.; Hirokoshi, T.; Nakajima, A. Adsorption of uranium by chitin phosphate and chitosan phosphate. Agric. Biol. Chem. 1981, 45, 2191-2195.

[74] a) Heras, A. ; Rodriguez, N.M. ; Ramos, V.M.; Agullo, E. $N$-methylene phosphonic chitosan: a novel soluble derivative. Carbohydr. Polym. 2001, 44, 1-8. b) Matevosyan, G.L.; Yukha, Y.S.; Zavlin, P.M. Phosphorylation of chitosan. Russ. J. Gen. Chem. 2003, 73, 1725-1728.

[75] Jayakumar, R. ; Egawa, T.; Furuike, T. ; Nair, S.V. ; Tamura, H. Synthesis, characterization, and thermal properties of phosphorylated chitin for biomedical applications. Polym. Eng. Sci. 2009, 49, 844-849.

[76] Jayakumar, R. ; Nagahama, H. ; Furuike, T. ; Tamura H. Synthesis of phosphorylated chitosan by novel method and its characterization. Int. J. Biol. Macromol. 2008, 42, 335-339.

[77] Coleman RJ, Lawrie G, Lambert LK, Whittaker M, Jack KS, Grøndahl L. Phosphorylation of alginate: synthesis, characterization, and evaluation of in vitro mineralization capacity. Biomacromolecules 2011, 12, 889897.

[78] Bernkop-Schnürch, A.; Schwarz, V.; Steininger, S. Polymers with thiol groups: a new generation of mucoadhesive polymers? Pharm. Res. 1999, 16, 876-881.

[79] Bernkop-Schnürch, A. ; Hornof, M. ; Guggi, D. Thiolated chitosans. Eur. J. Pharm. Biopharm. 2004, 57, 917.

[80] Sarti, F. ; Bernkop-Schnürch, A. Chitosan and Thiolated Chitosan. Adv. Polym. Sci. 2011, 243, 93-110.

[81] Bernkop-Schnürch, A. ; Brandt, U. ; Clausen, A. Synthese und in Vitro Evaluierung von Chitosan-Cystein Konjugaten. Sci. Pharm. 1999, 67, 197-208.

[82] a) Bernkop-Schnürch, A. ; Hornof, M. ; Zoidl, T. Thiolated polymers - thiomers: modification of chitosan with 2-iminothiolane. Int. J. Pharm. 2003, 260, 229-237. b) Singh, R. ; Kats, L. ; Blatter, W.A. ; Lambert, J.M. Formation of $\mathrm{N}$-substituted 2-iminothiolanes when amino groups in proteins and peptides are modified by 2iminithiolane. Anal. Biochem. 1996, 236, 114-125.

[83] a) Kafedjiiski, K. ; Krauland, A.H. ; Hoffer, M.H. ; Bernkop-Schnürch, A. Synthesis and in vitro evaluation of a novel thiolated chitosan. Biomaterials 2005, 26, 819-826. b) Delprino, L. ; Giacomotti, M. ; Dosio, F. ; Brusa, P. ; Ceruti, M. ; Grosa, G. ; Cattel, L. Toxin-targeted design for anticancer therapy. I: Synthesis and biological evaluation of new thioimidate heterobifunctional reagents. J. Pharm. Sci. 1993, 82, 506-512.

[84] a) Millotti, G. ; Samberger, C. ; Fröhlich, E. ; Bernkop-Schnürch, A. Chitosan-graft-6-mercaptonicotinic acid: synthesis, characterization, and biocompatibility. Biomacromolecules 2009, 10, 3023-3027. b) Millotti, G. ; Samberger, C. ; Fröhlich, E. ; Sakloetsakun, D. ; Bernkop-Schnürch, A. Chitosan-4-mercaptobenzoic acid: synthesis and characterization of a novel thiolated chitosan. J. Mater. Chem. 2010, 20, 2432-2440. 
[85] a) Holappa, J. ; Nevalainen, T. ; Savolainen, J. ; Soininen, P. ; Elomaa, M. ; Safin, R. ; Suvanto, S. ; Pakkanen, T. ; Másson, M. ; Loftsson, T. ; Järvinen, T. Synthesis and Characterization of Chitosan N-Betainates Having Various Degrees of Substitution. Macromolecules 2004, 37, 2784-2789. b) Makuška, R. ; Gorochovceva, N. Regioselective grafting of poly(ethylene glycol) onto chitosan through C-6 position of glucosamine units. Carbohydr. Polym. 2006, 64, 319-327.

[86] a) Nishimura, S.; Kohgo, O. ; Kurita, K. ; Kuzuhara, H. Chemospecific manipulations of a rigid polysaccharide: syntheses of novel chitosan derivatives with excellent solubility in common organic solvents by regioselective chemical modifications. Macromolecules 1991, 24, 4745-4748. b) Kurita, K. ; Shimada, K. ; Nishiyama, Y. ; Shimojoh, M. ; Nishimura, S. Nonnatural branched polysaccharides: synthesis and properties of chitin and chitosan having $\alpha$-mannoside branches. Macromolecules 1998, 31, 4764-4769.

[87] a) Kurita, K. ; Yoshino, H. ; Yokota, K. ; Ando, M. ; Inoue, S. ; Ishii, S. ; Nishimura, S. Preparation of tosylchitins as precursors for facile chemical modifications of chitin. Macromolecules 1992, 25, 3786-3790. b) Kurita, K. ; Yoshino, H. ; Nishimura, S. ; Ishii, S. Preparation and biodegradability of chitin derivatives having mercapto groups. Carbohydr. Polym. 1993, 20, 239-245.

[88] a) Kurita, K. ; Sugita, K. ; Kodaira, N. ; Hirakawa, M. Yang. J. Preparation and evaluation of trimethylsilylated chitin as a versatile precursor for facile chemical modifications. Biomacromolecules 6, 14141418. b) Kurita, K. ; Yoshida, Y.; Umemura, T. Finely selective protections and deprotections of multifunctional chitin and chitosan to synthesize key intermediates for regioselective chemical modifications. Carbohydr. Polym. 2010, 81, 434-440.

[89] Kolb, H.C. ; Finn, M.G. ; Sharpless, K.B. Click Chemistry: Diverse Chemical Function from a Few Good Reactions. Angew. Chem. Int. Ed. 2001, 40, 2004-2021.

[90] a) Kushwaha, D. ; Dwivedi, P. ; Kuanar, S.K. ; Tiwari, V.K. Click reaction in carbohydrate chemistry: recent developments and future perspective. Curr. Org. Synth. 2013, 10, 90-135. b) Elchinger, P.-H. ; Faugeras, P.-A.; Boëns, B. ; Brouillette, F. ; Montplaisir, D. ; Zerrouki, R. ; Lucas, R. Polysaccharides: the "click" chemistry impact. Polymers 2011, 3, 1607-1651. c) van Dijk, M. ; Rijkers, D.T.S. ; Liskamp, R.M.J. ; van Nostrum, C.F. ; Hennink, W.E. Synthesis and applications of biomedical and pharmaceutical polymers via click chemistry methodologies. Bioconjugate Chem. 2009, 20, 2001-2016.

[91] a) Tornøe, C.W. ; Christensen, C. ; Meldal, M. Peptidotriazoles on solid phase: [1,2,3]-triazole by regiospecific copper(I)-catalyzed 1,3-dipolar cycloadditions of terminal alkynes to azides. J. Org. Chem. 2002, 67, 3057-64. b) Rostovtsev, V.V.; Green, L.G.; Fokin, V.V.; Sharpless, K.B. A stepwise Huisgen cycloaddition process: copper(I)-catalyzed regioselective "ligation" of azides and terminal alkynes. Angew. Chem. Int. Ed. 2002, 41, 2596-2599.

[92] Zhang, L. ; Chen, X. ; Xue, P. ; Sun, H.H.Y. ; Williams, I.D. ; K. Barry Sharpless, K.B. ; Fokin, V.V. ; Jia, G. Ruthenium-Catalyzed Cycloaddition of Alkynes and Organic Azides. J. Am. Chem. Soc. 2005, 127, 1599815999.

[93] Agard, N.J. ; Prescher, J.A. ; Bertozzi, C.R. A Strain-Promoted [3 + 2] Azide-Alkyne Cycloaddition for Covalent Modification of Biomolecules in Living Systems. J. Am. Chem. Soc. 2004, 126, 15046-15047.

[94] Lallana, E. ; Fernandez-Megia, E. ; Riguera, R. Surpassing the Use of Copper in the Click Functionalization of Polymeric Nanostructures: A Strain-Promoted Approach. J. Am. Chem. Soc. 2009, 131, 5748-5750. 
[95] Baskin, J.M. ; Prescher, J.A. ; Laughlin, S.T. ; Agard, N.J. ; Chang, P.V. ; Miller, I.A. ; Lo, A. Codelli, J.A.; Bertozzi, C.R. Copper-free click chemistry for dynamic in vivo imaging. Proc. Natl. Acad. Sci. U.S.A. 2007, 104, 16793-16797.

[96] Van Berkel, S.S. ; Dirks, A.T. ; Debets, M.F. ; van Delft, F.L. ; Cornelissen, J.J.L.M. ; Nolte, R.J.M. ; Rutjes, F.P.J.T. Metal-free triazole formation as a tool for bioconjugation. Chem. Bio. Chem. 2007, 8, 15041508 .

[97] Sumerlin, B.S.; Vogt, A.P. Macromolecular engineering through click chemistry and other efficient transformations. Macromolecules 2010, 43, 1-13.

[98] a) Lutz, J.F. Copper-free azide-alkyne cycloadditions : New insights and perspectives. Angew. Chem. Int. Ed. 2008, 47, 2182-2184. b) Jewett, J.C.; Bertozzi, C.R. Cu-free click cycloaddition reactions in chemical biology. Chem. Soc. Rev. 2010, 39, 1272-1279

[99] Gress, A.; Vlkel, A.; Schlaad, H. Thio-click modification of poly[2-(3-butenyl)-2-oxazoline]. Macromolecules 2007, 40, 7928-33.

[100] Massi, A. ; Nanni, D. Thiol-yne coupling: revisiting old concepts as a breakthrough for up-to-date applications. Org. Biomol. Chem. 2012, 10, 3791-3807.

[101] Zhang, F. ; Bernet, B. ; Bonnet, V. ; Dangles, O. ; Sarabia, F. ; Vasella, A. 2-Azido-2-deoxycellulose: Synthesis and 1,3-Dipolar Cycloaddition. Helv. Chim. Acta 2008, 91, 608-617.

[102] Kaiser, E. ; Colescott, R. L. ; Bossinger, C. D. ; Cook, P. I. Color test for detection of free terminal amino groups in the solid-phase synthesis of peptides. Anal. Biochem. 1970, 34, 595-598.

[103] Cavender, C. J. ; Shiner, V. J. Trifluoromethanesulfonyl azide. Its reaction with alkyl amines to form alkyl azides. J. Org. Chem. 1972, 37, 3567-3569.

[104] Kulbokaite, R. ; Ciuta, G. ; Netopilik, M. ; Makuska, R. N-PEG'ylation of chitosan via “click chemistry” reactions. React. Funct. Polym. 2009, 69, 771-778.

[105] Peng, P. ; Cao, X. ; Peng, F. ; Bian, J. ; Xu, F. ; Sun, R. Binding cellulose and chitosan via click chemistry : synthesis, characterization, and formation of some hollow tubes. J. Polym. Sci. Part A 2012, 50, 5201-5210.

[106] Lallana, E. ; Fernandez-Megia, E. ; Riguera, R. Surpassing the use of copper in the click functionalization of polymeric nanostructures : a strain-promoted approach. J. Am. Chem. Soc. 2009, 131, 5748-5750.

[107] Ifuku, S.; Wada, M.; Morimoto, M.; Saimoto, H. Preparation of highly regioselective chitosan derivatives via "click chemistry". Carbohydr. Polym. 2011, 85, 653-657.

[108] Satoh, T. ; Kano, H. ; Nakatani, M. ; Nobuo Sakairi, N. ; Shinkai, S. ; Nagasaki, T. 6-Amino-6-deoxychitosan. Sequential chemical modifications at the C-6 positions of N-phthaloyl-chitosan and evaluation as a gene carrier. Carbohydr. Res. 2006, 341, 2406-2413.

[109] Chen, Y. ; Wang, F. ; Yun, D. ; Guo, Y. ; Ye, Y. ; Wang, Y. ; Tan, H. Preparation of a C-6 quaternary ammonium chitosan derivative through a chitosan Schiff base with Click Chemistry. J. Appl. Polym. Sci. 2013, 129, 3185-3191.

[110] Zampano, G. ; Bertoldo, M. ; Ciardelli, F. Defined chitosan-based networks by C-6-azide-alkyne "click" reaction. React. Funct. Polym. 2010, 70, 272-281. 
[111] Jirawutthiwongchai, J. ; Krause, A. ; Draeger, G. ; Chirachanchai, S. Chitosan-oxanorbornadiene : a convenient chitosan derivative for click chemistry without metal catalyst problem. ACS Macro Lett. 2013, 2, 177-180.

[112] Krause, A. ; Kirschning, A. ; Dräger, G. Bioorthogonal metal-free click-ligation of cRGD-pentapeptide to alginate. Org. Biomol. Chem. 2012, 10, 5547-5553.

[113] a) Lagos, A.; Reyes, J. Grafting onto chitosan. I. Graft copolymerization of methyl methacrylate onto chitosan with Fenton's reagent $\left(\mathrm{Fe}^{2+}-\mathrm{H}_{2} \mathrm{O}_{2}\right)$ as a redox initiator. J. Polym. Sci. Part Polym. Chem. 1988, 26, $985-$ 991. b) Kurita, K.; Kawata, M.; Koyama, Y.; Nishimura, S.-I. Graft copolymerization of vinyl monomers onto chitin with cerium (IV) ion. J. Appl. Polym. Sci. 1991, 42, 2885-2891. c) Wei Li; Zhaoyang Li; Wenshen Liao; Feng, X.-D. Chemical modification of biopolymers-mechanism of model graft copolymerization of chitosan. $J$. Biomater. Sci. Polym. Ed. 1993, 4, 557-566. d) Kojima, K.; Yoshikuni, M.; Suzuki, T. Tributylborane-initiated grafting of methyl methacrylate onto chitin. J. Appl. Polym. Sci. 1979, 24, 1587-1593.

[114] Yazdani-pedram, M.; Lagos, A.; Campos, N.; Retuert, J. Comparison of Redox Initiators Reactivities in the Grafting of Methyl Methacrylate onto Chitin. Int. J. Polym. Mater. 1992, 18, 25-37.

[115] a) Blair, H. S.; Guthrie, J.; Law, T.-K.; Turkington, P. Chitosan and modified chitosan membranes I. Preparation and characterisation AIBN. J. Appl. Polym. Sci. 1987, 33, 641-656. b) Yazdani-Pedram, M.; Retuert, J. Homogeneous grafting reaction of vinyl pyrrolidone onto chitosan. J. Appl. Polym. Sci. 1997, 63, 1321-1326.

[116] Hsu, S.-C.; Don, T.-M.; Chiu, W.-Y. Free radical degradation of chitosan with potassium persulfate. Polym. Degrad. Stab. 2002, 75, 73-83.

[117] a) Kurita, K.; Hashimoto, S.; Ishii, S.; Mori, T. Chitin/poly(methyl methacrylate) hybrid materials. Polym. Bull. 1996, 36, 681-686. b) Kurita, K.; Hashimoto, S.; Yoshino, H.; Ishii, S.; Nishimura, S.-I. Preparation of Chitin/Polystyrene Hybrid Materials by Efficient Graft Copolymerization Based on Mercaptochitin. Macromolecules 1996, 29, 1939-1942.

[118] Kurita, K.; Yoshino, H.; Nishimura, S.-I.; Ishii, S. Preparation and biodegradability of chitin derivatives having mercapto groups. Carbohydr. Polym. 1993, 20, 239-245.

[119] Liu, Y.; Liu, Z.; Zhang, Y.; Deng, K. Graft copolymerizaztion of methyl acrylate onto chitosan initiated by potassium diperiodatocuprate (III). J. Appl. Polym. Sci. 2003, 89, 2283-2289.

[120] Kurita, K.; Inoue, S.; Yamamura, K.; Yoshino, H.; Ishii, S.; Nishimura, S. Cationic and radical graft copolymerization of styrene onto iodochitin. Macromolecules 1992, 25, 3791-3794.

[121] Kurita, K.; Yoshino, H.; Yokota, K.; Ando, M.; Inoue, S.; Ishii, S.; Nishimura, S. Preparation of tosylchitins as precursors for facile chemical modifications of chitin. Macromolecules 1992, 25, 3786-3790.

[122] Ng, L.-T.; Guthrie, J. T.; Yuan, Y. J.; Zhao, H. UV-cured natural polymer-based membrane for biosensor application. J. Appl. Polym. Sci. 2001, 79, 466-472.

[123] Singh, V.; Tripathi, D. N.; Tiwari, A.; Sanghi, R. Microwave promoted synthesis of chitosan-graftpoly(acrylonitrile). J. Appl. Polym. Sci. 2005, 95, 820-825.

[124] Pengfei, L.; Maolin, Z.; Jilan, W. Study on radiation-induced grafting of styrene onto chitin and chitosan.

Radiat. Phys. Chem. 2001, 61, 149-153. b) Singh, D. K.; Ray, A. R. Graft copolymerization of 2hydroxyethylmethacrylate onto chitosan films and their blood compatibility. J. Appl. Polym. Sci. 1994, 53, 
1115-1121. c) Singh, D. K.; Ray, A. R. Radiation-induced grafting of N,N'-dimethylaminoethylmethacrylate onto chitosan films. J. Appl. Polym. Sci. 1997, 66, 869-877. d) Yu, L.; He, Y.; Bin, L.; Yue'e, F. Study of radiation-induced graft copolymerization of butyl acrylate onto chitosan in acetic acid aqueous solution. J. Appl. Polym. Sci. 2003, 90, 2855-2860.

[125] Qu, X.; Wirsén, A.; Albertsson, A.-C. Structural change and swelling mechanism of pH-sensitive hydrogels based on chitosan and D,L-lactic acid. J. Appl. Polym. Sci. 1999, 74, 3186-3192.

[126] Feng, H.; Dong, C.-M. Synthesis and characterization of phthaloyl-chitosan-g-poly(l-lactide) using an organic catalyst. Carbohydr. Polym. 2007, 70, 258-264.

[127] Chung, T. W.; Yang, J.; Akaike, T.; Cho, K. Y.; Nah, J. W.; Kim, S. I.; Cho, C. S. Preparation of alginate/galactosylated chitosan scaffold for hepatocyte attachment. Biomaterials 2002, 23, 2827-2834.

[128] a) Jenkins, D. W.; Hudson, S. M. Review of Vinyl Graft Copolymerization Featuring Recent Advances toward Controlled Radical-Based Reactions and Illustrated with Chitin/Chitosan Trunk Polymers. Chem. Rev. 2001, 101, 3245-3274. b) Kurita, K.; Iwawaki, S.; Ishii, S.; Nishimura, S.-I. Introduction of poly(L-alanine) side chains into chitin as versatile spacer arms having a terminal free amino group and immobilization of nadh active sites. J. Polym. Sci. Part Polym. Chem. 1992, 30, 685-688. c) Salamone, J. C. Polymeric materials encyclopedia; CRC Press: Boca Raton, 1996; Vol. 2.

[129] Detchprohm, S.; Aoi, K.; Okada, M. Synthesis of a Novel Chitin Derivative Having Oligo(e-caprolactone) Side Chains in Aqueous Reaction Media. Macromol. Chem. Phys. 2001, 202, 3560-3570.

[130] a) Aoi, K.; Takasu, A.; Okada, M. Synthesis of novel chitin derivatives having poly(2-alkyl-2-oxazoline) side chains. Macromol. Chem. Phys. 1994, 195, 3835-3844. b) Aoi, K.; Takasu, A.; Okada, M. New ChitinBased Polymer Hybrids. 2. Improved Miscibility of Chitin Derivatives Having Monodisperse Poly(2-methyl-2oxazoline) Side Chains with Poly(vinyl chloride) and Poly(vinyl alcohol)1. Macromolecules 1997, 30, 61346138. c) Aoi, K.; Takasu, A.; Okada, M. Miscibility of poly(vinyl chloride) with chitin derivatives having poly(2-methyl-2-oxazoline) side chains. Macromol. Rapid Commun. 1995, 16, 53-58. d) Sato, H.; Tsuge, S.; Ohtani, H.; Aoi, K.; Takasu, A.; Okada, M. Characterization of Chitin-Based Polymer Hybrids by TemperatureProgrammed Analytical Pyrolysis Techniques. 1. Chitin-graft-poly(2-methyl-2-oxazoline)/Poly(vinyl chloride) Blends. Macromolecules 1997, 30, 4030-4037.

[131] a) Aoi, K.; Takasu, A.; Okada, M.; Imae, T. Synthesis and assembly of novel chitin derivatives having amphiphilic polyoxazoline block copolymer as a side chain. Macromol. Chem. Phys. 1999, 200, 1112-1120. b) Yoshikawa, S.; Takayama, T.; Tsubokawa, N. Grafting reaction of living polymer cations with amino groups on chitosan powder. J. Appl. Polym. Sci. 1998, 68, 1883-1889.

[132] Odian, G. Principles of Polymerization; Wiley \& Sons: New York, 2004.

[133] Gorocheva, N.; Kulbokaite, R.; Makuska, R.; Judkenas, R. Synthesis and study of chitosan and poly(ethylene glycol) graft copolymers containing triazine moiety. Chemija 2004, 15, 22-27

[134] Gorochovceva, N.; Makuška, R. Synthesis and study of water-soluble chitosan-O-poly(ethylene glycol) graft copolymers. Eur. Polym. J. 2004, 40, 685-691.

[135] Ouchi, T.; Nishizawa, H.; Ohya, Y. Aggregation phenomenon of PEG-grafted chitosan in aqueous solution. Polymer 1998, 39, 5171-5175.

[136] Saito, H.; Wu, X.; Harris, J. M.; Hoffman, A. S. Graft copolymers of poly(ethylene glycol) (PEG) and chitosan. Macromol. Rapid Commun. 1997, 18, 547-550. 
[137] Harris, J. M.; Struck, E. C.; Case, M. G.; Paley, M. S.; Yalpani, M.; Van Alstine, J. M.; Brooks, D. E. Synthesis and characterization of poly(ethylene glycol) derivatives. J. Polym. Sci. Polym. Chem. Ed. 1984, 22, $341-352$.

[138] Harris, J. M.; Sedaghat-Herati, M. R. United States Patent: 5252714 - Preparation and use of polyethylene glycol propionaldehyde. 5252714, October 12, 1993.

[139] Bentley, M. D.; Roberts, M. J.; Harris, J. M. Reductive amination using poly(ethylene glycol) acetaldehyde hydrate generated in situ: Applications to chitosan and lysozyme. J. Pharm. Sci. 1998, 87, 14461449.

[140] Silva, S. S.; Menezes, S. M. C.; Garcia, R. B. Synthesis and characterization of polyurethane-g-chitosan. Eur. Polym. J. 2003, 39, 1515-1519.

[141] Kawamura, Y.; Mitsuhashi, M.; Tanibe, H.; Yoshida, H. Adsorption of metal ions on polyaminated highly porous chitosan chelating resin. Ind. Eng. Chem. Res. 1993, 32, 386-391.

[142] Hoffman, A. S.; Chen, G. Graft copolymers of PEO-PPO-PEO triblock polyethers on bioadhesive polymer backbones: Synthesis and properties. Polym Prepr 1997, 38, 524-525.

[143] Kweon, D. K. Preparation and characteristics of chitosan-g-PDMS copolymer. Polym. Bull. 1998, 41, 645-651.

[144] Kim, I. Y.; Kim, S. J.; Shin, M.-S.; Lee, Y. M.; Shin, D.-I.; Kim, S. I. pH- and thermal characteristics of graft hydrogels based on chitosan and poly(dimethylsiloxane). J. Appl. Polym. Sci. 2002, 85, 2661-2666.

[145] a) Shah, S. B.; Patel, C. P.; Trivedi, H. C. Fenton's reagent-initiated graft copolymerization of acrylonitrile onto sodium alginate. J. Appl. Polym. Sci. 1994, 51, 1421-1426. b) Shah, S. B.; Patel, C. P.; Trivedi, H. C. Kinetics and reaction mechanism of fenton's-reagent-initiated graft copolymerization of acrylonitrile onto sodium alginate. J. Appl. Polym. Sci. 1994, 52, 857-860.

[146] Pawar, S. N.; Edgar, K. J. Alginate derivatization: A review of chemistry, properties and applications. Biomaterials 2012, 33, 3279-3305.

[147] Shah, S. B.; Patel, C. P.; Trivedi, H. C. Ceric-induced grafting of acrylate monomers onto sodium alginate. Carbohydr. Polym. 1995, 26, 61-67.

[148] Isıklan, N.; Kursun, F. Synthesis and characterization of graft copolymer of sodium alginate and poly(itaconic acid) by the redox system. Polym. Bull. 2013, 70, 1065-1084.

[149] Isıklan, N.; Kursun, F.; Inal, M. Graft copolymerization of itaconic acid onto sodium alginate using benzoyl peroxide. Carbohydr. Polym. 2010, 79, 665-672.

[150] Sadeghi, M.; Nahid, G.; Fatemeh, S. Optimization of Synthetic Conditions of a Novel Graft Copolymer Based on Alginate. Eur. J. Sci. Res. 2011, 64, 587-597.

[151] Shi, X.; Wang, W.; Kang, Y.; Wang, A. Enhanced swelling properties of a novel sodium alginate-based superabsorbent composites: NaAlg-g-poly(NaA-co-St)/APT. J. Appl. Polym. Sci. 2012, 125, 1822-1832.

[152] a) Hua, S.; Wang, A. Synthesis, characterization and swelling behaviors of sodium alginate-g-poly(acrylic acid)/sodium humate superabsorbent. Carbohydr. Polym. 2009, 75, 79-84. b) Wang, W.; Wang, A. Synthesis and swelling properties of $\mathrm{pH}$-sensitive semi-IPN superabsorbent hydrogels based on sodium alginate-gpoly(sodium acrylate) and polyvinylpyrrolidone. Carbohydr. Polym. 2010, 80, 1028-1036.

[153] Sand, A.; Yadav, M.; Behari, K. Synthesis and characterization of alginate-g-vinyl sulfonic acid with a potassium peroxydiphosphate/thiourea system. J. Appl. Polym. Sci. 2010, 118, 3685-3694. 
[154] Yadav, M.; Mishra, D. K.; Sand, A.; Behari, K. Modification of alginate through the grafting of 2acrylamidoglycolic acid and study of physicochemical properties in terms of swelling capacity, metal ion sorption, flocculation and biodegradability. Carbohydr. Polym. 2011, 84, 83-89.

[155] Sand, A.; Yadav, M.; Mishra, D. K.; Behari, K. Modification of alginate by grafting of N-vinyl-2pyrrolidone and studies of physicochemical properties in terms of swelling capacity, metal-ion uptake and flocculation. Carbohydr. Polym. 2010, 80, 1147-1154.

[156] Liu, Y. H.; Li, Y.; Yang, L.; Liu, Y.; Bai, L. Graft copolymerization of methyl acrylate onto sodium alginate initiated by potassium diperiodatocuprate(III). Polymery 2005, 50, 37-42.

[157] Sen, G.; Singh, R. P.; Pal, S. Microwave-initiated synthesis of polyacrylamide grafted sodium alginate: Synthesis and characterization. J. Appl. Polym. Sci. 2010, 115, 63-71.

[158 ]Sorour, M.; El-Sayed, M.; Moneem, N. A. E.; Talaat, H. A.; Shalaan, H.; Marsafy, S. E. Characterization of hydrogel synthesized from natural polysaccharides blend grafted acrylamide using microwave (MW) and ultraviolet (UV) techniques. Starch - Stärke 2013, 65, 172-178

[159] Mollah, M. Z. I.; Khan, M. A.; Hoque, M. A.; Aziz, A. Studies of physico-mechanical properties of photocured sodium alginate with silane monomer. Carbohydr. Polym. 2008, 72, 349-355.

[160] Ju, H. K. ; Kim, S.Y. ; Lee, Y.M. pH/temperature-responsive behaviors of semi-IPN and comb-type graft hydrogels compose dog alginate and poly(N-isopolyacrylamide). Polymer 2001, 42, 6851-6857.

[161] a) Athawale, V. D.; Padwaldesai, M. P. Free radical graft copolymerization of methacrylamide onto agar. Eur. Polym. J. 1999, 35, 1237-1243. b) Athawale, V. D.; Padwaldesai, M. P. Copolymerization of methacrylic acid with agar using $\mathrm{Ce}+4$ as initiator. J. Polym. Mater. 2000, 17, 1-7.

[162] Prasad, K.; Mehta, G.; Meena, R.; Siddhanta, A. K. Hydrogel-forming agar-graft-PVP and kappacarrageenan-graft-PVP blends: Rapid synthesis and characterization. J. Appl. Polym. Sci. 2006, 102, 3654-3663. [163] a) Rani, G. U.; Mishra, S.; Sen, G.; Jha, U. Polyacrylamide grafted Agar: Synthesis and applications of conventional and microwave assisted technique. Carbohydr. Polym. 2012, 90, 784-791. b) Mishra, S.; Sen, G.; Rani, G. U.; Sinha, S. Microwave assisted synthesis of polyacrylamide grafted agar (Ag-g-PAM) and its application as flocculant for wastewater treatment. Int. J. Biol. Macromol. 2011, 49, 591-598.

[164] Pourjavadi, A.; Harzandi, A. M.; Hosseinzadeh, H. Modified carrageenan 3. Synthesis of a novel polysaccharide-based superabsorbent hydrogel via graft copolymerization of acrylic acid onto kappacarrageenan in air. Eur. Polym. J. 2004, 40, 1363-1370.

[165] Pourjavadi, A.; Barzegar, S.; Zeidabadi, F. Synthesis and properties of biodegradable hydrogels of $\kappa-$ carrageenan grafted acrylic acid-co-2-acrylamido-2-methylpropanesulfonic acid as candidates for drug delivery systems. React. Funct. Polym. 2007, 67, 644-654.

[166] Mishra, D. K.; Tripathy, J.; Behari, K. Synthesis of graft copolymer (k-carrageenan-g-N,Ndimethylacrylamide) and studies of metal ion uptake, swelling capacity and flocculation properties. Carbohydr. Polym. 2008, 71, 524-534.

[167] a) Yadav, M.; Sand, A.; Mishra, M. M.; Tripathy, J.; Pandey, V. S.; Behari, K. Synthesis, characterization and applications of graft copolymer (K-carrageenan-g-vinylsulfonic acid). Int. J. Biol. Macromol. 2012, 50, 826832. b) Sand, A.; Mishra, D. K.; Pandey, V. S.; Mishra, M. M.; Behari, K. Synthesis of graft copolymer $(\mathrm{CgOH}-$ g-AGA): Physicochemical properties, characterization and application. Carbohydr. Polym. 2012, 90, 901-907. 
c) Mishra, M. M.; Yadav, M.; Sand, A.; Tripathy, J.; Behari, K. Water soluble graft copolymer (K-carrageenang-N-vinyl formamide): Preparation, characterization and application. Carbohydr. Polym. 2010, 80, $235-241$.

[168] Mishra, M. M.; Sand, A.; Mishra, D. K.; Yadav, M.; Behari, K. Free radical graft copolymerization of Nvinyl-2-pyrrolidone onto k-carrageenan in aqueous media and applications. Carbohydr. Polym. 2010, 82, 424431.

[169] El-Mohdy, H. L. A.; El-Rehim, H. A. A. Radiation-induced kappa carrageenan/acrylic acid graftcopolymers and their application as catalytic reagent for sucrose hydrolysis. Chem. Eng. J. 2008, 145, 154-159. [170] Furusaki, E.; Ueno, Y.; Sakairi, N.; Nishi, N.; Tokura, S. Facile preparation and inclusion ability of a chitosan derivative bearing carboxymethyl- $\beta$-cyclodextrin. Carbohydr. Polym. 1996, 29, 29-34.

[171] Aoki, N.; Nishikawa, M.; Hattori, K. Synthesis of chitosan derivatives bearing cyclodextrin and adsorption of p-nonylphenol and bisphenol A. Carbohydr. Polym. 2003, 52, 219-223.

[172] a) Tojima, T.; Katsura, H.; Han, S.-M.; Tanida, F.; Nishi, N.; Tokura, S.; Sakairi, N. Preparation of an acyclodextrin-linked chitosan derivative via reductive amination strategy. J. Polym. Sci. Part Polym. Chem. 1998, 36, 1965-1968. b) Tanida, F.; Tojima, T.; Han, S.-M.; Nishi, N.; Tokura, S.; Sakairi, N.; Seino, H.; Hamada, K. Novel synthesis of a water-soluble cyclodextrin-polymer having a chitosan skeleton. Polymer 1998, 39, 52615263.

[173] Prabaharan, M.; Mano, J. F. Chitosan derivatives bearing cyclodextrin cavitiesas novel adsorbent matrices. Carbohydr. Polym. 2006, 63, 153-166.

[174] Wade, L. G. In Organic Chemistry; $4^{\text {th }}$ ed.; Prentice-Hall International, Inc.; Englewood Cliffs, New Jersey, 1999 ; pp. 822-836.

[175] Martel, B.; Devassine, M.; Crini, G.; Weltrowski, M.; Bourdonneau, M.; Morcellet, M. Preparation and sorption properties of a $\beta$-cyclodextrin-linked chitosan derivative. J. Polym. Sci. Part Polym. Chem. 2001, 39, 169-176.

[176] Chen, S.; Wang, Y. Study on $\beta$-cyclodextrin grafting with chitosan and slow release of its inclusion complex with radioactive iodine. J. Appl. Polym. Sci. 2001, 82, 2414-2421.

[177] Zhang, X.; Wang, Y.; Yi, Y. Synthesis and characterization of grafting $\beta$-cyclodextrin with chitosan. J. Appl. Polym. Sci. 2004, 94, 860-864.

[178] Gaffar, M. A.; El-Rafie, S. M.; El-Tahlawy, K. F. Preparation and utilization of ionic exchange resin via graft copolymerization of $\beta$-CD itaconate with chitosan. Carbohydr. Polym. 2004, 56, 387-396.

[179] Peng, C.; Wang, Y.; Tang, Y. Synthesis of crosslinked chitosan-crown ethers and evaluation of these products as adsorbents for metal ions. J. Appl. Polym. Sci. 1998, 70, 501-506.

[180] Tang, X.-H.; Tan, S.-Y.; Wang, Y.-T. Study of the synthesis of chitosan derivatives containing benzo-21crown-7 and their adsorption properties for metal ions. J. Appl. Polym. Sci. 2002, 83, 1886-1891.

[181] Wan, L.; Wang, Y.; Qian, S. Study on the adsorption properties of novel crown ether crosslinked chitosan for metal ions. J. Appl. Polym. Sci. 2002, 84, 29-34.

[182] a) Ding, S.; Zhang, X.; Feng, X.; Wang, Y.; Ma, S.; Peng, Q.; Zhang, W. Synthesis of N,N'-diallyl dibenzo 18-crown-6 crown ether crosslinked chitosan and their adsorption properties for metal ions. React. Funct. Polym. 2006, 66, 357-363. b) Zhang, X.; Ding, S.; Wang, Y.; Feng, X.; Peng, Q.; Ma, S. Synthesis and 
adsorption properties of metal ions of novel azacrown ether crosslinked chitosan. J. Appl. Polym. Sci. 2006, 100, 2705-2709.

[183] Radwan, A. A.; Alanazi, F. K.; Alsarra, I. A. Microwave Irradiation-Assisted Synthesis of a Novel Crown Ether Crosslinked Chitosan as a Chelating Agent for Heavy Metal Ions (M+n). Molecules 2010, 15, 6257-6268.

[184] Tabakci, M.; Yilmaz, M. Synthesis of a chitosan-linked calix[4]arene chelating polymer and its sorption ability toward heavy metals and dichromate anions. Bioresour. Technol. 2008, 99, 6642-6645.

[185] Pluemsab, W.; Sakairi, N.; Furuike, T. Synthesis and inclusion property of $\alpha$-cyclodextrin-linked alginate. Polymer 2005, 46, 9778-9783.

[186] Wilchek, M. ; Jakoby, W.B. The literature on affinity chromatography. Methods Enzymol. B 1974, 34, 310.

[187] Blanco-Fernandez, B. ; Lopez-Viota, M. ; Concheiro, A. ; Alvarez-Lorenzo, C. Synergistic performance of cyclodextrin-agar hydrogels for ciprofloxacin delivery and antimicrobial effect. Carbohydr. Polym. 2011, 85, $765-774$.

[188] Bar R. A new cyclodextrin-agar medium for surface cultivation of microbes on lipophilic substrates. Appl. Microbiol. Biotechnol. 1990, 32, 470-472.

[189] Tsubokawa, N.; Takayama, T. Surface modification of chitosan powder by grafting of "dendrimer-like" hyperbranched polymer onto the surface. React. Funct. Polym. 2000, 43, 341-350.

[190] a) Sashiwa, H.; Shigemasa, Y.; Roy, R. Chemical Modification of Chitosan. 3. Hyperbranched ChitosanSialic Acid Dendrimer Hybrid with Tetraethylene Glycol Spacer. Macromolecules 2000, 33, 6913-6915. b) Sashiwa, H.; Shigemasa, Y.; Roy, R. Chemical Modification of Chitosan. 10.1 Synthesis of Dendronized Chitosan-Sialic Acid Hybrid Using Convergent Grafting of Preassembled Dendrons Built on Gallic Acid and Tri(ethylene glycol) Backbone. Macromolecules 2001, 34, 3905-3909. c) Sashiwa, H.; Shigemasa, Y.; Roy, R. Highly Convergent Synthesis of Dendrimerized Chitosan-Sialic Acid Hybrid1. Macromolecules 2001, 34, 32113214. d) Sashiwa, H.; Shigemasa, Y.; Roy, R. Chemical modification of chitosan 8: preparation of chitosandendrimer hybrids via short spacer. Carbohydr. Polym. 2002, 47, 191-199. e) Sashiwa, H.; Shigemasa, Y.; Roy, R. Chemical modification of chitosan 11: chitosan-dendrimer hybrid as a tree like molecule. Carbohyd. Polym. 2002, 49, 195-205. f) Sashiwa, H.; Yajima, H.; Aiba, S. Synthesis of a Chitosan-Dendrimer Hybrid and Its Biodegradation. Biomacromolecules 2003, 4, 1244-1249.

[191] Qu, R. ; Sun, C. ; Ji, C. ; Wang, C. ; Chen, H. ; Nieu, Y. ; Liang, C. ; Song, Q. Preparation and metalbinding behavior chitosa functionalized by ester- and amino-terminated hyperbanched polyamidoamine polymers. Carbohydr. Res. 2008, 343, 267-273.

[192] a) Kurita, K.; Kanari, M.; Koyama, Y. Studies on chitin. Polym. Bull. 1985, 14, 511-514. b) Kurita, K.; Yoshida, A.; Koyama, Y. Studies on chitin. 13. New polysaccharide/polypeptide hybrid materials based on chitin and poly(.gamma.-methyl L-glutamate). Macromolecules 1988, 21, 1579-1583.

[193] Chung, T.-W.; Lu, Y.-F.; Wang, S.-S.; Lin, Y.-S.; Chu, S.-H. Growth of human endothelial cells on photochemically grafted Gly-Arg-Gly-Asp (GRGD) chitosans. Biomaterials 2002, 23, 4803-4809.

[194] Suzuki, Y.; Tanihara, M.; Suzuki, K.; Saitou, A.; Sufan, W.; Nishimura, Y. Alginate hydrogel linked with synthetic oligopeptide derived from BMP-2 allows ectopic osteoinduction in vivo. J. Biomed. Mater. Res. 2000, $50,405-409$. 
[195] Zhu, H.; Ji, J.; Lin, R.; Gao, C.; Feng, L.; Shen, J. Surface engineering of poly(DL-lactic acid) by entrapment of alginate-amino acid derivatives for promotion of chondrogenesis. Biomaterials 2002, 23, 31413148 .

[196] Fonseca, K. B.; Bidarra, S. J.; Oliveira, M. J.; Granja, P. L.; Barrias, C. C. Molecularly designed alginate hydrogels susceptible to local proteolysis as three-dimensional cellular microenvironments. Acta Biomater. 2011, 7, 1674-1682.

[197] Bubenikova, S.; Stancu, I.-C.; Kalinovska, L.; Schacht, E.; Lippens, E.; Declercq, H.; Cornelissen, M.; Santin, M.; Amblard, M.; Martinez, J. Chemoselective cross-linking of alginate with thiol-terminated peptides for tissue engineering applications. Carbohydr. Polym. 2012, 88, 1239-1250.

[198] Oza, M. D.; Prasad, K.; Siddhanta, A. K. One-pot synthesis of fluorescent polysaccharides: adenine grafted agarose and carrageenan. Carbohydr. Res. 2012, 357, 23-31. b) Oza, M. D.; Meena, R.; Siddhanta, A. K. Facile synthesis of fluorescent polysaccharides: Cytosine grafted agarose and א-carrageenan. Carbohydr. Polym. 2012, 87, 1971-1979.

[199] Berger, J.; Reist, M.; Mayer, J. M.; Felt, O.; Peppas, N. A.; Gurny, R. Structure and interactions in covalently and ionically crosslinked chitosan hydrogels for biomedical applications. Eur. J. Pharm. Biopharm. 2004, 57, 19-34.

[200] a) Giri, T. K.; Thakur, A.; Alexander, A.; Ajazuddin; Badwaik, H.; Tripathi, D. K. Modified chitosan hydrogels as drug delivery and tissue engineering systems: present status and applications. Acta Pharm. Sin. B 2012, 2, 439-449. b) Li, Y.; Rodrigues, J.; Tomás, H. Injectable and biodegradable hydrogels: gelation, biodegradation and biomedical applications. Chem. Soc. Rev. 2012, 41, 2193-2221. c) Ravi Kumar, M. N. . A review of chitin and chitosan applications. React. Funct. Polym. 2000, 46, 1-27. d) Augst, A. D.; Kong, H. J.; Mooney, D. J. Alginate Hydrogels as Biomaterials. Macromol. Biosci. 2006, 6, 623-633. e) Lee, K. Y.; Mooney, D. J. Hydrogels for Tissue Engineering. Chem. Rev. 2001, 101, 1869-1880.

[201] a) Dal Pozzo, A.; Vanini, L.; Fagnoni, M.; Guerrini, M.; De Benedittis, A.; Muzzarelli, R. A. A. Preparation and characterization of poly(ethylene glycol)-crosslinked reacetylated chitosans. Carbohydr. Polym. 2000, 42, 201-206. b) Kim, S. S.; Lee, Y. M.; Cho, C. S. Synthesis and properties of semi-interpenetrating polymer networks composed of $\beta$-chitin and poly(ethylene glycol) macromer. Polymer 1995, 36, 4497-4501. c) Crescenzi, V.; Paradossi, G.; Desideri, P.; Dentini, M.; Cavalieri, F.; Amici, E.; Lisi, R. New hydrogels based on carbohydrate and on carbohydrate-synthetic polymer networks. Polym. Gels Networks 1997, 5, 225-239. d) Crescenzi, V.; Imbriaco, D.; Velàsquez, C. L.; Dentini, M.; Ciferri, A. Novel types of polysaccharidic assemblies. Macromol. Chem. Phys. 1995, 196, 2873-2880.

[202] a) Kim, S. S.; Kim, H. W.; Yuk, S. H.; Oh, S. Y.; Pak, P. K.; Lee, H. B. Blood and cell compatibility of gelatin-carrageenan mixtures cross-linked by glutaraldehyde. Biomaterials 1996, 17, 813-821. b) Meena, R.; Prasad, K.; Siddhanta, A. K. Development of a stable hydrogel network based on agar-kappa-carrageenan blend cross-linked with genipin. Food Hydrocoll. 2009, 23, 497-509.

[203] Verma, V.; Verma, P.; Kar, S.; Ray, P.; Ray, A. R. Fabrication of agar-gelatin hybrid scaffolds using a novel entrapment method for in vitro tissue engineering applications. Biotechnol. Bioeng. 2007, 96, 392-400.

[204] a) DeKosky, B. J.; Dormer, N. H.; Ingavle, G. C.; Roatch, C. H.; Lomakin, J.; Detamore, M. S.; Gehrke, S. H. Hierarchically Designed Agarose and Poly(Ethylene Glycol) Interpenetrating Network Hydrogels for Cartilage Tissue Engineering. Tissue Eng. Part C Methods 2010, 16, 1533-1542. b) Pescosolido, L.; 
Vermonden, T.; Malda, J.; Censi, R.; Dhert, W. J. A.; Alhaique, F.; Hennink, W. E.; Matricardi, P. In situ forming IPN hydrogels of calcium alginate and dextran-HEMA for biomedical applications. Acta Biomater. 2011, 7, 1627-1633. c) Sen, M.; Avci, E. N. Radiation synthesis of poly(N-vinyl-2-pyrrolidone)-kappacarrageenan hydrogels and their use in wound dressing applications. I. Preliminary laboratory tests. J. Biomed. Mater. Res. A 2005, 74A, 187-196.

[205] Jing, R.; Yanqun, Z.; Jiuqiang, L.; Hongfei, H. Radiation synthesis and characteristic of IPN hydrogels composed of poly(diallyldimethylammonium chloride) and Kappa-Carrageenan. Radiat. Phys. Chem. 2001, 62, $277-281$.

[206] Berger, J.; Reist, M.; Mayer, J.M.; Felt, O.; Gurny, R. Structure and interactions in chitosan hydrogels formed by complexation or aggregation for biomedical applications. Eur. J. Pharm. Biopharm. 2004, 57, 35-52. [207] a) Mi, F.-L.; Tan, Y.-C.; Liang, H.-F.; Sung, H.-W. In vivo biocompatibility and degradability of a novel injectable-chitosan-based implant. Biomaterials 2002, 23, 181-191. b) Rao, B. S.; Murthy, K. V. R. Preparation and In Vitro Evaluation of Chitosan Matrices Cross-Linked by Formaldehyde Vapors. http://informahealthcare.com/doi/abs/10.1081/DDC-100100272 (accessed September 9, 2013). c) Sano, M.; Hosoya, O.; Taoka, S.; Seki, T.; Kawaguchi, T.; Sugibayashi, K.; Juni, K.; Morimoto, Y. Relationship between Solubility of Chitosan in Alcoholic Solution and Its Gelation. Chem. Pharm. Bull. (Tokyo) 1999, 47, 1044-1046. d) Thacharodi, D.; Rao, K. P. Development and in vitro evaluation of chitosan-based transdermal drug delivery systems for the controlled delivery of propranolol hydrochloride. Biomaterials 1995, 16, 145-148.

[208] Klug, S.; Merker, H.-J.; Jäckh, R. Effects of ethylene glycol and metabolites on in vitro development of rat embryos during organogenesis. Toxicol. In Vitro 2001, 15, 635-642. 